\title{
CAMA
}

Centre for Applied Macroeconomic Analysis

\section{Financial Conditions and the Risks to Economic Growth in the United States Since 1875}

\section{CAMA Working Paper 36/2020 April 2020}

\author{
Patrick J. Coe
}

Carleton University

Centre for Applied Macroeconomic Analysis, ANU

\section{Shaun P. Vahey}

University of Warwick

Centre for Applied Macroeconomic Analysis, ANU

\begin{abstract}
We explore the historical relationship between financial conditions and real economic growth for quarterly U.S. data from 1875 to 2017 with a flexible empirical copula modelling methodology. We compare specifications with both linear and non-linear dependence, and with both Gaussian and non-Gaussian marginal distributions. Our results indicate strong statistical support for models that are both non-Gaussian and nonlinear for our historical data, with considerable heterogeneity across sub-samples. We demonstrate that ignoring the contribution of financial conditions typically understates the conditional downside risks to economic growth in crises. For example, accounting for financial conditions more than doubles the probability of negative growth in the year following the 1929 stock market crash.
\end{abstract}




\title{
Keywords
}

Probabilities of economic events, Vulnerable growth, Growth at risk, Great Depression.

\author{
JEL Classification
}

C14, C32, C53, E37, E44, N10, N20

\section{Address for correspondence:}

(E) cama.admin@anu.edu.au

ISSN 2206-0332

The Centre for Applied Macroeconomic Analysis in the Crawford School of Public Policy has been established to build strong links between professional macroeconomists. It provides a forum for quality macroeconomic research and discussion of policy issues between academia, government and the private sector.

The Crawford School of Public Policy is the Australian National University's public policy school, serving and influencing Australia, Asia and the Pacific through advanced policy research, graduate and executive education, and policy impact. 


\title{
Financial Conditions and the Risks to Economic Growth in the United States Since 1875*
}

\author{
Patrick J. Coe \\ Carleton University \\ and CAMA (ANU)
}

\author{
Shaun P. Vahey \\ University of Warwick \\ and CAMA (ANU)
}

First draft: September 27, 2019

This draft: April 16, 2020

\begin{abstract}
We explore the historical relationship between financial conditions and real economic growth for quarterly U.S. data from 1875 to 2017 with a flexible empirical copula modelling methodology. We compare specifications with both linear and non-linear dependence, and with both Gaussian and non-Gaussian marginal distributions. Our results indicate strong statistical support for models that are both non-Gaussian and non-linear for our historical data, with considerable heterogeneity across sub-samples. We demonstrate that ignoring the contribution of financial conditions typically understates the conditional downside risks to economic growth in crises. For example, accounting for financial conditions more than doubles the probability of negative growth in the year following the 1929 stock market crash.
\end{abstract}

Keywords: Probabilities of economic events; Vulnerable growth; Growth at risk; Great Depression.

JEL codes: C14; C32; C53; E37; E44; N10; N20

${ }^{*}$ We thank Todd Clark, Domenico Giannone, Dean Croushore, Simon van Norden, Catherine Doz, Laurent Ferrara, Sylvia Kaufmann, Edward Anderson, and seminar participants at the University of Sydney, the Canadian Network for Economic History Conference, 2019, and the Banque de France and Paris School of Economics Macroeconometrics and Time Series Workshop, 2019. A previous version of this manuscript circulated with the title "Financial Conditions and Vulnerable Growth, 1875-2017". The not for publication appendix can be downloaded from shaunvahey.com. 


\section{Introduction}

Some important recent empirical macroeconomic research highlights the impact of financial conditions on the risks to economic growth. Papers by, among others, Adrian, Boyarchenko and Giannone (2019a, ABG) and Adrian et al (2018) argue that economic growth has a nonGaussian distribution. Their research quantifies the vulnerability of economic growth using quantile regression methods and provides the basis for the IMF's bilateral surveillance tool, "growth at risk".

Earlier studies finding strong empirical linkages between the severity of recessions and financial crisis using historical data include, for example, Bordo and Haubrich (2010), Schularick and Taylor (2012), Jordà, Schularick and Taylor (2013, 2016) and Jalil (2015). These papers build on a considerable volume of historical qualitative analysis emphasizing the complexity of economic growth during episodes of financial crisis.

In this paper, we take a broad historical perspective of the relationship between financial conditions and real economic growth using quarterly U.S. data from 1875 to 2017. Given the emphasis in the historical literature on the complexity of economic growth, we compute probabilistic assessments utilizing a copula modelling strategy. Our methodology has sufficient flexibility to encompass both non-Gaussianity and non-linearity.

Looking across sub-samples of historical U.S. data and across a variety of measures of financial conditions and economic growth, and considering both Root Mean Squared Error and a KLIC-based measure of probabilistic predictive content, we find financial conditions matter for economic growth. We also find that there is strong statistical support for both non-Gaussianity and non-linearity. Furthermore, restricting attention to linear dependence limits the predictive content from financial conditions, regardless of whether or not the fitted marginal distributions are Gaussian. These results hold across all our sub-samples, even though there is considerable heterogeneity across historical eras. 
We consider the impact of financial conditions on the probabilities of various economic growth events based on our preferred non-Gaussian and non-linear copula specification. We find that ignoring the contribution of financial conditions typically understates the conditional downside risks to economic growth in crises. For example, including financial conditions more than doubles the risk of negative economic growth from 31 percent to 79 percent for the year following the 1929 stock market crash.

In terms of our modelling methodology, we build on recent time series research adopting copula methods to separate margins from dependence. Following, among others, Smith (2015), Smith and Vahey (2016), Loaiza-Maya and Smith (2019) and Karagedikli, Vahey and Wakerly (2019), we fit non-parametric marginal distributions based on the Empirical Cumulative Distribution Function (ECDF) for each macroeconomic variable. In this manner, we provide scope for non-Gaussianity in the macroeconomic variables, while modelling both cross-sectional and serial dependence in the copula density.

Unlike these previous macroeconomic copula studies, we adopt an empirical copula methodology, fitting the dependence parameters by deploying a kernel density estimator, with adjustments for boundaries, using non-parametric estimation on pseudo data. In contrast to our approach, direct kernel density estimation based on the observed time series would combine any non-Gaussianity and non-linearity; DiNardo and Tobias (2001) and the density impulse responses of Adrian, Boyarchenko and Giannone (2019b) provide examples of that approach.

In addition to ABG (2019a), Chavleishvili and Manganelli (2019), Ferrara, Mogliani, and Sahuc (2019) and Loria, Matthes and Zhang (2019) all adopt (modified) quantile regression methods to study the importance of non-Gaussianity for economic growth with modern era data. Bernard and Czada (2015) discuss robustness issues for dependence estimates delivered by quantile regression methods in the presence of non-Gaussianity. Amengual, Sentana and Tian (2019) propose robust methods to compute linear dependence with copulas. (We adapt their approach to estimate a specification with linear dependence and non-Gaussian distributed 
variables.) Carriero, Clark and Marcellino (2019) note that the modern era U.S. data contain relatively few pertinent observations to assess tail behaviour for output growth. Historical data give further scope to study predictability for economic growth events.

In summary, we clarify the complex historical relationship between financial conditions and economic growth by modelling non-linearity and non-Gaussianity separately. Both features matter for probabilistic economic growth assessments based on financial conditions with historical data.

The remainder of this paper is structured as follows. In Section 2, we set out our macroeconomic copula modelling methodology, describing how the approach has sufficient flexibility to separate non-Gaussian and non-linear features for the variables explored by ABG (2019a). In Section 3, we summarize the U.S. historical data used, exploring variations in the measures of financial conditions and economic growth by sub-sample. We consider the same National Financial Conditions Index (NFCI) for modern data as ABG (2019a), a factor model based index of over 100 indicators of financial conditions, in addition to our other measures of financial conditions. In Section 4, we present our prediction results, probabilistic assessments of the downside and upside risks to economic growth, together with discussions of the implications for selected financial crises. In the final section, we conclude by discussing briefly a future avenue for research utilizing non-Gaussian and non-linear models of economic growth.

\section{Macroeconomic Copula Modelling}

By utilizing empirical copula modelling methods, we can distinguish between non-Gaussianity and non-linearity. A copula model separates the margins from the dependence structure, facilitating estimation of a robust, distribution-free measure of dependence based on ranks.

In this section, first we describe how the copula modelling methodology separates margins from dependence and then we give a detailed account of our approach to fitting the empirical 
copula models to macroeconomic data. We conclude this section by describing prediction and evaluation.

\subsection{Separation of Margins and Dependence}

To show how copulas help distinguish between non-Gaussian and non-linear features in general, we exploit Skars' Theorem (Nelsen, 2006) to decompose a joint distribution into univariate margins and dependence.

Suppose we consider a multivariate joint distribution for $S$ variables stacked in $\boldsymbol{Z}$, with a (row) vector of time series observations for each variable. Sklar's Theorem indicates that a unique copula exists, $C$, under certain regularity conditions, which separates the joint distribution into the univariate margins and the dependence structure. We express the joint distribution function for $\boldsymbol{Z}$ using the copula function, $C$ :

$$
F(\boldsymbol{z})=C(\boldsymbol{u})
$$

where $\boldsymbol{z}=\left(\boldsymbol{z}_{1}^{\prime}, \ldots, \boldsymbol{z}_{S}^{\prime}\right)^{\prime}$ and $\boldsymbol{u}=\left(\boldsymbol{u}_{1}^{\prime}, \ldots, \boldsymbol{u}_{S}^{\prime}\right)^{\prime}$. The joint distribution on the right hand side, $C$, is defined on the $S$-dimensional unit cube, $[0,1]^{S}$. The $S$ variables in $\boldsymbol{u}$ are often referred to as "copula data". Denoting the Cumulative Distribution Function, $F(\cdot)$, we can define the copula data as $\boldsymbol{u}_{s}=F_{s}\left(\boldsymbol{z}_{s}\right)$ so that they are individually uniformly distributed for each margin.

We differentiate the distribution function, equation (1), to give the probability density of $\boldsymbol{Z}$ as the product of the copula density and the $S$ marginal densities:

$$
f(\boldsymbol{z})=c(\boldsymbol{u}) \prod_{s=1}^{S} f_{s}\left(\boldsymbol{z}_{s}\right)
$$

where $f_{s}\left(\boldsymbol{z}_{s}\right)$ is the marginal density of $\boldsymbol{z}_{s}$ and $c(\boldsymbol{u})=\frac{\partial}{\partial \boldsymbol{u}} C(\mathbf{u})$ is the copula density.

With the copula density representing the dependence on the unit cube, and non-linearity 
being a property of the dependence, non-Gaussianity is captured separately by the margins.

\section{$2.2 \quad$ Fitting Empirical Copulas to Macroeconomic Variables}

Each of the $S$ macroeconomic variables refers to a time series of length $T$ with corresponding copula data $\boldsymbol{u}_{s}=\left(u_{s, 1}, \ldots, u_{s, T}\right)^{\prime}$. We assume that the time series variables are stationary; see Smith (2015) for a discussion of stationarity and copula modelling.

The Probability Integral Transforms provide computationally convenient copula data derived from the Empirical Cumulative Distribution Function (ECDF) for each variable. Previous applications working with ECDF marginal distributions on modern macroeconomic data include Smith and Vahey (2016), Loaiza-Maya and Smith (2019) and Karagedikli, Vahey and Wakerly (2019).

In this paper, we adopt a flexible route to fitting dependence by adapting empirical copula methods. In contrast, Smith and Vahey (2016), Loaiza-Maya and Smith (2019) and Karagedikli, Vahey and Wakerly (2019) fit parametric copula densities. Empirical copula models have both non-parametric marginals and a non-parametric copula density; see Deheuvals (1979), Deheuvals (1981) and, for a recent example, Velásquez-Giraldo et al (2018).

Empirical copula studies typically utilize rank-transformed counterparts to the copula data, sometimes referred to as "pseudo data", to mitigate the danger of misspecification in fitting the marginal distributions. For each macroeconomic variable, indexed by $s$, we define the pseudo data as $V_{s, t}=R_{s, t} /(T+1)$, where for each variable, $R$ denotes the rank of the $t^{\text {th }}$ observation relative to its own history, for $t=1, \ldots, T$. The $T+1$ denominator avoids boundary issues.

We consider a similar model space to ABG (2019a) with three macroeconomic variables, so that $S=3$. Namely, output growth and lagged financial conditions, with lagged output growth also included as a predetermined conditioning variable. We fit the copula density non-parametrically to the three (rank-transformed) pseudo variables to capture dependence. 
With the dependence estimates conditional on the fitted marginals, this limited information estimation strategy is sometimes known as "inference for margins"; see, Joe (2006).

We fit the multivariate pseudo time series using a kernel density estimator (KDE). Among others, Silverman (1986), Scott (1992), DiNardo and Tobias (2001), Li and Racine (2006) and Adrian, Boyarchenko and Giannone (2019b) adopt KDE methods but not within a copula framework. We utilise a Beta kernel to fit the multivariate distribution on the $S$-dimensional unit cube, reducing scope for boundary bias; see the discussions in Chen (1999), Charpentier, Fermanian and Scaillet (2007) and Karra (2018). The fitted copula density is:

$$
\frac{1}{T} \sum_{t=1}^{T} \prod_{s=1}^{3} K\left(V_{s, t}, \alpha, \beta\right)
$$

where $K$ denotes the Beta density with parameters $\alpha=\frac{v}{h}+1$ and $\beta=\frac{1-v}{h}+1$ and $h$ denotes bandwidth. Following Adrian, Boyarchenko and Giannone (2019b), we select the bandwidth for our KDE based on out of sample performance for the modern era. We utilize the same bandwidth value for the historical data prior to 1970. We describe our process in detail in the not for publication appendix.

Mindful of the complexity of economic growth in financial crises previously documented by economic historians, the capacity of our empirical copula methodology to handle both nonGaussianity and non-linearity is appealing. Nevertheless, there is no reason to rule out either Gaussianity or linearity a priori. Hence, we fit four specifications in total. First, a benchmark model with Gaussian marginals and linear dependence - a copula model equivalent to a conventional linear-Gaussian regression, with linear dependence estimated by maximum likelihood. Second, maintaining the assumption of linearity for dependence, we consider non-Gaussian marginals, with estimation by maximum likelihood based on the pseudo data. Third, we consider non-linear dependence, but adopt Gaussian marginals. And, for the final specification, the most general model, we consider non-Gaussian marginals with non-linear dependence. 


\section{$2.3 \quad$ Prediction and Evaluation}

Prediction involves simulation directly from the copula density on the unit cube for all four specifications. Then, we exploit the appropriate fitted marginal for each specification to produce predictions on the observable scale for output growth. The last step uses the inverse marginal for output growth, $F_{1}^{-1}(\cdot)$, to generate predictions with the required distribution for the target variable, output growth. We deploy the inverse ECDF for the two non-Gaussian specifications and the inverse Normal for the two Gaussian specifications. ${ }^{1}$

Turning to the assessment of predictive accuracy for our four specifications, we examine both relative Root Mean Squared Errors (RMSE) and a relative entropy based measure of probabilistic accuracy. The RMSE approach computes the square root of the average of squared errors from the conditional mean prediction for each specification, with the lowest RMSE preferred. The idea behind the relative entropy approach is to select a specification which on average gives highest probability to the data. A Bayesian interpretation of this Kullback-Leibler Information Criterion (KLIC) approach to model selection is given by Fernandez-Villaverde and Rubio-Ramirez (2004). See, for further details, the discussions in Kullback and Leibler (1951), Kullback (1987), and Roberston, Tallman and Whiteman (2005). As noted by, for example, Kascha and Ravazzolo (2010), (under some regularity conditions) KLIC optimization is equivalent to minimizing the average logarithmic score (log score) of the densities using the sample data.

\section{Data}

Given the emphasis in the economic history literature on looking at evidence across a variety of sub-samples and measures of macroeconomic variables, we describe the data considerations

\footnotetext{
${ }^{1}$ Therefore, for the specification with linear dependence and non-Gaussian marginals, the implied correlation between observables exhibits (slight) non-linearity as a result of the non-linear transform associated with the inverse ECDF.
} 
in detail. To facilitate comparisons with ABG (2019a), we examine their preferred measure of financial conditions for modern data and, in addition, we explore other measures of financial conditions germane for our historical sub-samples. We also consider similar measures of economic growth to ABG (2019a), albeit using GNP rather than GDP to give a consistent series across historical sub-samples.

Reflecting the heterogeneity in the scale of economic fluctuations through our $100+$ year span of quarterly data, and following much of the historical literature, we divide our sample into four sub-samples and estimate our empirical models for each era. The first of these, 1875:1 to 1913:4, covers the classical Gold Standard. The second, 1919:1 to 1941:3, begins just after World War I and ends just prior to the U.S. entry into World War II. ${ }^{2}$ The third era, 1946:1 to 1971:2, corresponds to the Bretton Woods exchange rate system. The fourth era, 1971:3 to 2017:4, starts with the demise of the Bretton Woods system and includes both the Great Moderation and the 2008 financial crisis. Bordo and Haubrich (2010), Jordà et al (2013, 2016) and Schularick and Taylor (2012) use similar era dates. ${ }^{3}$ ABG (2019a) consider the most recent (modern) era only.

We follow ABG (2019a) in reporting results for both annualized one and four quarter growth rates of economic growth. We collected our quarterly GNP data from 1875 to 2017 using the FRED database and Balke and Gordon (1986). The length of the sub-samples varies by era, with 154, 90, 101 and 185 observations used for estimation with the one quarter measure, and 148, 87, 98 and 182 used with the four quarter measure. For our first three eras we used the real GNP series from Balke and Gordon to construct the growth measures, and for our last era we used the real GNP series from the FRED database. Further details of the sources and construction of all variables are available in the not for publication appendix.

\footnotetext{
${ }^{2}$ We find very similar results when our second era spans 1914:1 to 1945:4 and so includes both world wars. These results are reported in the not for publication appendix.

${ }^{3}$ In the not for publication appendix we allow marginal distributions to vary by era, but impose a common dependence structure. As with the results reported in the main text, we find that the addition of financial conditions significantly improves in-sample fit.
} 
Since the Chicago Fed's National Financial Conditions Index (NCFI) only covers the modern sub-sample from the early 1970s, we explored alternative measures of financial conditions for all eras. These are the credit spread, the term spread and a measure of stock market volatility. For the first era, we used Railway Bond yields from the NBER Macrohistory database, reported by Macaulay (1938), to construct a credit spread. For the remaining three eras, we used the Baa-Aaa spread calculated from FRED data. For term spreads in our first three eras, we followed Balke and Gordon (1986), using Railway Bond Yields and the Moody's Baa rated bonds to construct a long-term corporate bond yield, and subtract from that the 60-90 day New York City Commercial Paper rate. These data are from FRED and Macaulay (1938). For our final era, we used the difference between the 10-Year Treasury Constant Maturity Rate and the 3-Month Treasury Bill rate to represent the term spread, with both series from FRED. Finally, we used Shiller's S\&P Composite Index to measure stock market volatility for the four sub-samples. ${ }^{4}$

Table 1 provides summary statistics for both the one quarter (upper panel) and four quarter measure (lower panel) of output growth by era. The first two rows (in each panel) refer to the mean and standard deviation of the sub-samples, respectively. These illustrate the differences in scale across eras, regardless of the measure of output growth, with variation in the unconditional means and standard deviations. The first era has the highest unconditional mean at 4.08 and the second era has the highest standard deviation at around 12.5. The second era has the lowest unconditional mean at 2.75. This is similar to the mean for the modern sub-sample, 2.77. The modern era has the lowest standard deviation at 3.22. Therefore, while the sub-sample including the Great Moderation and the Great Recession has seen relative stability in economic growth, mean growth is close to that of the sub-sample that includes the Great Depression. ${ }^{5}$

\footnotetext{
${ }^{4}$ Post-2000 data from Shiller (2000) can be downloaded from http://www.econ.yale.edu/ shiller/data.htm.

${ }^{5}$ Romer (1989) notes differences in construction methods for GNP across eras.
} 
The remaining rows display summary statistics for skewness, kurtosis, and the $p$-values from the Shaprio-Wilk test for non-Gaussianity. All eras exhibit negative skew based on the one quarter measure of growth. Three of the four eras have excess kurtosis, indicating heavy tails in the sub-samples. The second era is an exception with kurtosis close to three - the value for a Gaussian distribution. The Shapiro-Wilk test indicates significant deviations from Gaussianity for all sub-samples, although for the second era the $p$-value is 13 percent.

Turning to the summary statistics for the four quarter measure of economic growth displayed in the lower panel of Table 1, the general patterns are similar to those with the one quarter measure, but with lower values for the standard deviation and kurtosis for each era. Negative skew predominates with this measure, and the $p$-values for the Shapiro-Wilk test are generally below 5 percent. An exception is the four quarter economic growth measure for the third era, Bretton Woods, which has very slightly positive skew.

The summary statistics for our various measures of financial conditions are reported in Table 2. In general, the financial conditions exhibit positive skew, but the term spread during the first and last sub-samples displays negative skew. Generally, there is excess kurtosis across these measures of financial conditions, with the term spread an exception. Finally, the $p$-values for the Shaprio-Wilk test are below 10 percent and in most cases they are below 1 percent, implying a rejection of the null hypothesis of Gaussianity, with the term spread in the first era an exception.

Overall, looking across measures of output growth and financial conditions, and subsamples, there is evidence of non-Gaussianity for both variables - motivating our consideration of non-Gaussian marginal distributions in our subsequent analysis. 


\section{Results}

In this section, we analyse the evidence supporting both non-Gaussianity and non-linearity, and assess whether financial conditions have predictive content for economic growth in our various historical sub-samples. Then, we present our conditional output growth predictive densities and consider the probabilities of output growth events, with an emphasis on wellknown historical financial crises. We begin with an ocular assessment of the fitted marginal distributions by era. This provides further support for non-Gaussianity in general but highlights the complex and varied forms of departures from Gaussianity across eras, particularly for measures of financial conditions.

\subsection{Fitted Marginal Distributions}

Figure 1 provides eight plots displaying the fitted marginal distributions (plotted as probability density functions, smoothed for illustration) from the ECDFs for output growth for each era. ${ }^{6}$ We provide results for various economic growth measures, with plots based on the one quarter GNP growth measure in the left column, and the four quarter measure in the right column. Each row of plots refers to a specific sub-sample.

Looking at the top left panel, namely one quarter growth during the Gold Standard era, we see the density exhibits an asymmetric distribution, with negative skew overall, a slightly longer and thinner left tail, and a slightly shorter and fatter right tail. Turning to the modern sub-sample, post-1970, we see a similar pattern in the bottom left panel. ABG (2019a) noted the considerable probability mass to the right of the mode, reflecting the long upswing of the business cycle. Looking at the third row, Bretton Woods, we again see similar features. However, the second era, which includes the Great Depression, has a distinct almost triangular

\footnotetext{
${ }^{6}$ The locally-adaptive univariate KDE developed by Shimazaki and Shinomoto (2010) was used for all results described below.
} 
shape indicating considerable probability mass to the left of the mode. Put differently, U.S. output growth was unusually "vulnerable" during the years between the wars, which includes the Great Depression. In general, though, the shapes of the densities across eras (rows) and output growth measures (left and right columns) are similar (ignoring the obvious differences in scale).

Moving onto the marginals for financial conditions, Figure 2 provides the corresponding densities for a variety of measures. The four rows of plots refer to the respective eras, ordered by start date, with (for example) the modern era shown in the bottom row. The three columns from left to right refer to the term spread, the credit spread and S\&P volatility, respectively. Looking across eras and measures, all plots display features that are hard to reconcile with Gaussian distributions. Nevertheless, there is heterogeneity across measures for each era. For example, for the first row, the Gold Standard era, the credit spread and S\&P volatility both have a long thin right tail, whereas the term spread has a relatively short (but fat) right tail with a longer and thinner left tail. Recall that the overall skew for the term spread measure in this era is slightly negative. Although the term spread and S\&P volatility are unimodal, the credit spread has a second (local) peak at around 0.5. Generally the credit spread and S\&P volatility have similar shapes for each era, with the term spread more idiosyncratic from an ocular perspective.

Overall, we conclude from our ocular assessment that the fitted marginal distrbibutions are typically non-Gaussian but in complex ways that are not easy to reconcile with parametric distributions, especially for measures of financial conditions.

\subsection{Assessing Predictive Content}

Tables 3 and 4 summarize the in-sample fit of our various specifications using RMSE and the KLIC-based measure of predictive performance, respectively. Although we focus in particular 
on the additional predictive content from (lagged) financial conditions, recall that throughout we condition on lagged output growth - matching the variables of interest in ABG (2019a).

Each of the panels displayed in Tables 3 and 4 corresponds to a single specification. The first panel considers the baseline specification with Gaussian marginals and linear dependenceequivalent to a conventional linear-Gaussian regression. The second panel refers to the specification with non-Gaussian marginals and linear dependence. The third panel covers the case with Gaussian marginals and non-linear dependence. And, the fourth panel refers to the most general specification, with non-Gaussian marginals and non-linear dependence. Rows within each panel consider the four measures of financial conditions displayed below the row referring to the variant without financial conditions. The eight columns are based on specific sub-samples, with separate results for each measure of output growth.

Looking at Table 3, reporting the relative RMSE of our various specifications, we see from the first panel that adding financial conditions adds little to the fit of the benchmark linear-Gaussian specification. Generally, the gains from including this variable are less than two percent, regardless of the financial conditions measure, and regardless of the measure of output growth.

One exception is the measure of the financial conditions emphasized by ABG (2019a), the Chicago Fed NFCI, reported in the bottom row of the first panel, where the gains are around five percent of RMSE relative to the benchmark for the modern era. ${ }^{7}$ A second exception is the term spread, for the first sub-sample for both output growth measures.

Turning to the second panel of Table 3, we see fairly small gains in predicability from adopting non-Gaussian marginals with linear dependence. Without financial conditions, RMSE is close to the linear-Gaussian benchmark. And, adding financial conditions rarely makes much difference to predictability over the corresponding cases in the previous panel or the benchmark. For example, considering the Chicago Fed NFCI, the gains are again close to five percent

\footnotetext{
${ }^{7}$ Recall that the NFCI was introduced after the 2008 crisis and currently refers to post-1970 data only.
} 
relative to the benchmark for both measures of output growth in the modern era.

In contrast, for the third panel of Table 3, we see much larger predictability gains. With Gaussian marginals and non-linear dependence, but ignoring financial conditions, the improvement in RMSE is under 10 percent, regardless of sub-sample or measure of output growth. However, there are considerable gains from including financial conditions for all measures and eras. For example the predictive gain from including the term spread is around 20 percent during the Gold Standard era for the one quarter economic growth measure (relative to the benchmark). For the subsequent eras, the corresponding gains are up to 30 percent for the same measure of output growth, depending on the measure of financial conditions. For the four quarter measure, the gains are in the region of 20 to 40 percent with some variation across eras. Considering the Chicago Fed NFCI, the gain is approximately 15 to 30 percent for both measures of output growth in the modern era. Overall, we conclude that there is strong evidence for the inclusion of financial conditions in all sub-samples, with non-linear dependence and Gaussian marginals. And weak evidence that non-linearity matters even if we do not consider financial conditions.

Considering the fourth panel of Table 3, we see stronger evidence for non-Gaussianity in conjunction with non-linear dependence. Even the case without financial conditions is consistently better than the Gaussian-linear benchmark. Across all sub-samples and for all measures of output growth, relative RMSEs are below one, in the first row of the fourth panel, with gains in the region of one to 11 percent. Turning to the issue of whether financial conditions matter for this more general specification, we see large predictive gains for all measures and eras in the fourth panel of Table 3. The predictive gain from including, for example, the credit spread is around 20 percent for the Gold Standard era and the one quarter economic growth measure. For the subsequent eras, the gains are as much as 40 percent for the same measures of output growth and financial conditions; see, for example, the second era (including the Great Depression) where the gain is around 42 percent. Similar gains in 
predictability from including the financial variables are displayed across all sub-samples for the four quarter measure of output growth. For the Chicago Fed NFCI variable, the gain in predictability is in the region of 25 to 30 percent relative to the benchmark.

Overall, we have three main findings based on the results presented in Table 3. First, in terms of ranking the predictability of our various specifications, the non-Gaussian and nonlinear model is best, followed by the Gaussian and non-linear specification. Second, there is strong evidence for the inclusion of financial conditions in all sub-samples with our preferred specification. Third, even in the specification with Gaussian marginals and non-linear dependence, including financial conditions matters for predictability. So much so, that without financial conditions there is only a modest performance gain from moving beyond the Gaussian-linear benchmark. These findings are robust across measures of output growth and measure of financial conditions.

Turning now to the consideration of the KLIC in Table 4, we draw similar results in terms of probabilistic predictability — of particular relevance for risk considerations. Regardless of the measure of output growth, including financial conditions (by any measure) improves predictability in our preferred non-Gaussian and non-linear specification. ${ }^{8}$ As with RMSE, the KLIC results indicate that Gaussian specifications with non-linear dependence rank second overall in terms of predictability if financial conditions are included. From a probabilistic perspective, there is a small predictability gain from adopting the Gaussian and non-linear specification over the benchmark in the absence of financial conditions.

\subsection{Conditional Output Growth Densities}

Figures 3a through 3d plot the means of the conditional distribution for output growth along with $5^{\text {th }}$ and $95^{\text {th }}$ percentiles over time and the data realizations. In all cases the conditional

\footnotetext{
${ }^{8}$ We report recursive prediction results for our preferred empirical copula specification (and the linear-Gaussian benchmark) in the not for publication appendix. These illustrate further the importance of financial conditions.
} 
distributions use our preferred empirical specification with non-Gaussian (ECDF) marginals and non-linear dependence. We provide results using the NFCI to measure financial conditions to facilitate comparison with ABG (2019a) for the modern era. We report results based on the credit spread measure of financial conditions for the interwar and Bretton Woods sub-samples. Empirical studies using the credit spread include Bernanke (1983), Coe (2002) and Bordo and Haubrich (2010) using historical data, together with Gertler and Koradi (2015) and Caldara and Herbst (2019) using more modern data. Since our credit spread measure is based on railway bonds only for the Gold Standard era, we focus on results based on the term spread from this era. See Bordo and Haubrich (2008) for a historical study exploring the relationship between the term spread and output growth. ${ }^{9}$

In the top row of Figrues 3a through $3 \mathrm{~d}$, we condition only on lagged output; and, for the bottom row, we condition on both lagged output growth and lagged financial conditions. The columns on the left contain plots for the one quarter growth measure and those on the right contain plots for the four quarter growth measure.

Two features are immediately striking when looking at these figures. First, with the addition of financial conditions, the conditional mean tracks the data much more closely, consistent with the large drop in RMSE reported in the last panel of Table 3. This characteristic emerges for realizations of output growth in the lower tail of the unconditional distribution, and also for realizations closer to the historical mean. Therefore, while our discussion below focuses on episodes considered as financial crises, our results suggest that financial conditions also contain information about movements in output growth near the central probability mass. Second, the difference between the $5^{\text {th }}$ and $95^{\text {th }}$ percentiles is much smaller in the lower panels-indicating reduced uncertainty when accounting for financial conditions. These features occur regardless of the measures of output growth.

\footnotetext{
${ }^{9}$ In terms of overall predictability, our results are robust to the choice of financial conditions measure; see the not for publication appendix.
} 
Looking more closely at Figure 3d, which refers to the modern era, the conditional mean declines around 2008 for both measures of output growth when including financial conditions in the bottom row. Furthermore, there is a coincident drop in the $5^{\text {th }}$ percentile - consistent with the conclusion that financial conditions contributed to increased growth vulnerabilities.

The Jordá, Schularick and Taylor (2017) Macrohistory Database identifies three crisis, in 1893, 1907 and 1929, that of particular interest to economic historians. Figures 3a and 3b display features for these crises resembling those for the 2008 crisis (in Figure 3d). Namely, coincident declines in the conditional mean and $5^{\text {th }}$ percentile for both one quarter and four quarters measures of economic growth. We explore the risks to economic growth in further detail in the next sub-section.

\subsection{Probabilities of Low and High Output Growth Events}

Figures 4a through 4d plot the probabilities of low and high growth events over time for our preferred specification with non-Gaussian (ECDF) marginals and non-linear dependence, conditioning on financial conditions (and lagged output growth). ${ }^{10}$ The choice of events is somewhat arbitrary in this approach. When looking at growth rates in the lower tail, Jordá et al (2017) use the bottom decile. We define a low growth event as output growth below the $10^{\text {th }}$ percentile, and we define a high growth event as above the $90^{\text {th }}$ percentile, based on the unconditional distribution for each era.

Figures 4a through 4d plot probabilities without financial conditions in the top row and provide probabilities with financial conditions in the bottom row. The plots in the left column indicate the probabilities of low and high growth in the next quarter, while the plots in the right column indicate the corresponding probabilities for the next year. In each panel,

\footnotetext{
${ }^{10}$ We use the same measures as in Figure 3. Corresponding plots for all measures of financial conditions are reported in the not for publication appendix, together with results for alternative definitions of low and high growth events.
} 
the probabilities of low growth are depicted by red bars, measured upwards from zero. The probabilities of high growth are depicted by blue bars, measured downwards from one.

Comparing the top and the bottom row for Figures 4a through 4d, respectively, financial conditions result in increased variation in the conditional probabilities, regardless of the measure of output growth. Looking more closely at the interwar era, for example, the probabilities of the low growth event are typically in the range of 0.1 to 0.2 , and only above 0.2 on a few occasions absent financial conditions. In particular, there are only modest increases in the conditional probability (to around 0.35) following the 1929 stock market crash and during the Great Depression. However, when we include financial conditions, these probabilities peak much higher following the 1929 crash and remain above 0.5 for much of the Great Depression.

Increased risk of low growth events also occurs during other financial crises when accounting for financial conditions. In the first era, for example, there are large increases in the probability of low growth events during the 1893 and 1907 crises displayed in Figure 4a. Figure 4d displays the higher probability of low growth during the Global Financial Crisis.

Recall that our empirical copula approach allows for non-Gaussianity in the conditional densities in both tails - giving the flexibility to track upside and downside risk, simultaneously. ABG (2019a) report that downside risks are related to financial conditions, but that upside risks are not. In contrast, our copula-based approach suggests a strong influence of financial conditions on both upside and downside risks to economic growth. Financial conditions sharpen the predictive densities for economic growth in each era, represented by Figures 4a through $4 \mathrm{~d}$.

\subsection{Selected Financial Crises}

Figure 5 plots the conditional predictive densities for output growth during four financial crises. The panels on the left refer to output growth in the quarter following the financial crisis and 
the panels on the right refer to output growth in the year following the financial crisis. These are predictive densities for future output growth conditional on current financial conditions and current output growth. Each row refers to a specific crisis. Namely, the second quarter of 1893, the fourth quarter of 1907, the fourth quarter of 1929 and the fourth quarter of 2008. In each panel, we plot the predictive densities with and without (lagged) financial conditions, for our preferred non-Gaussian and non-linear specification (conditioning on lagged output growth throughout).

The top row of Figure 5 plots the predictive densities for the 1893 crisis, associated with a fall in confidence regarding the Gold Standard. Arguably, the Sherman Silver Purchase Act of 1890 and the decline in Treasury gold reserves after December 1892 unsettled financial markets; see, for example, the discussion in Rockoff (1990). An alternative view, provided by Friedman and Schwartz (1963), argues that concerns over bank solvency led to bank runs. Remaining agnostics on this issue, we assume that the 1893 crisis occurred in the second quarter of 1893 to coincide with the decline in stock prices and the bank runs in June of that year. The top row of Figure 5 shows the predictive densities for output growth in both the subsequent quarter and year. ${ }^{11}$ For the one quarter measure of economic growth (left column), the conditional predictive density without financial conditions (in black) displays evidence of negative skew, with a mode slightly below zero. The corresponding predictive density with financial conditions (in red) exhibits bi-modality, with twin peaks in probability at approximately zero and -25 percent economic growth. For the year after the 1893 crisis, the twin peaks occur at approximately -10 and two percent economic growth. Disregarding financial conditions for the one year ahead case results in just a single probability peak at around seven percent economic growth. Financial conditions radically change the assessments of downside risks to economic growth around the 1893 crisis.

The second row of Figure 5 plots the predictive densities for the 1907 crisis, which arguably

\footnotetext{
${ }^{11}$ As for Figures 3 and 4 , we use the term spread to represent financial conditions in this row.
} 
originated with runs in the "shadow banking" sector; see, for example, Frydman et al (2015). According to Friedman and Schwartz (1963), the downturn began in May 1907 and was amplified by the failure of the Knickerbocker Trust in October, leading to a run on New York trust companies. With these companies being outside the New York Clearing House system they did not have access to the same funds as national banks. Arguably, the funds organized by J.P. Morgan, in part financed by the Treasury, played an important role in halting the crisis. Frydman et al (2015) draw a parallel between these funds and lending by the Federal Reserve during 2008. We assume that the 1907 crisis occurred in the fourth quarter of 1907 . The second row of Figure 5 shows the predictive densities with (red) and without (black) financial conditions for the subsequent quarter (left column) and year (right column). The predictive densities indicate higher probability of negative economic growth with financial conditions for both horizons at around 90 percent (one quarter) and 25 percent (four quarter). As with the 1983 crisis, there is evidence of bi-modality only when accounting for financial conditions.

The third row of Figure 5 plots the predictive densities for output growth following the 1929:4 stock market crash. At both the one quarter and year ahead horizons, the probability of negative output growth is markedly greater if we condition on financial conditions. For the one quarter ahead (left column), the probability of negative growth is nearly 100 percent. For the year ahead (right column) the corresponding probability is around 80 percent. Without financial conditions, the probabilities drop to around 70 percent and 30 percent, respectively. Interestingly, although the four quarter ahead predictive density is bi-modal, the one quarter ahead predictive is unimodal when accounting for financial conditions.

The bottom row of Figure 5 plots predictive densities for the fourth quarter of 2008. We use the same NFCI measure of financial conditions as ABG (2019a). ${ }^{12}$ The lower conditional predicted mean and the (previously noted) increase in downside risks for both the quarter and

\footnotetext{
${ }^{12}$ ABG (2019a) report similar results using a term spread, a credit spread and a measure of stock market volatility. We report qualitatively similar results in the not for publication appendix, along with results using alternative measures of financial conditions for other eras.
} 
the year following 2008:4 are apparent from the predictive densities with financial conditions (red) and without (black). However, the impact of financial conditions on downside risk are more modest for 2008 than for the earlier crises.

We emphasize the bi-modality evident in five out of the six cases when conditioning on financial conditions for the 1893, 1907 and 1929 crises. This property contributes considerably to the downside risk assessments during historical crises. Ignoring financial conditions results in weaker downside risk and typically unimodal probability density functions. Although financial conditions also influence the assessment of downside risk for the 2008 crisis, the effect is less pronounced and the predictive densities are unimodal. The historical evidence presented here suggests even stronger impacts of financial conditions on downside risk for economic growth than for the most recent crisis.

\section{Conclusion}

Our probabilistic analysis of the historical evidence supports the empirical connection between financial conditions and economic growth in the United States, building on earlier work by Bordo and Haubrich (2010), Schularick and Taylor (2012), Jordà, Schularick and Taylor (2013, 2016), Jalil (2015), Adrian, Boyarchenko and Giannone (2019a) and others. Our consideration of a variety of measures of economic growth and financial conditions establishes the robustness of the relationship across sub-samples of quarterly historical data from 1875 to 2017.

Our paper differs substantially from those in the extant literature. This study is the first to provide probabilistic assessments of the impact of financial conditions on both the downside and upside risks to economic growth for historical data. We find that both non-Gaussianity and non-linearity shape the historical relationship between these important macroeconomic variables.

Given the effectiveness of the NFCI as a predictor for economic growth on modern data 
documented by Adrian, Boyarchenko and Giannone (2019a), a corresponding index suitable for historical analysis would be helpful for subsequent research. This would permit probabilistic assessments for historical events based on a still wider set of financial conditions measures using non-Gaussian and non-linear specifications. 


\section{References}

Adrian, Tobias, Nina Boyarchenko, and Domenico Giannone (2019a) "Vulnerable Growth" American Economic Review 109(4) pp1263-1289.

Adrian, Tobias, Nina Boyarchenko, and Domenico Giannone (2019b) "Multimodality in MacroFinancial Dynamics" unpublished manuscript October.

Adrian, Tobias, Federico Grinberg, Nellie Liang, and Sheheryar Malik (2018) "The Term Structure of Growth-at-Risk" IMF Working Paper WP/18/180.

Amengual, Dante, Enrique Sentana and Zhanyuan Tian (2019) "Gaussian Rank Correlation and Regression" unpublished manuscript.

Balke, Nathan S. and Robert J. Gordon (1986) "Appendix B: Historical Data" in Robert J. Gordon ed. The American Business Cycle: Continuity and Change University of Chicago Press, Chicago.

Bernanke, Ben S. (1983) "Nonmonetary Effects of the Financial Crisis in the Propagation of the Great Depression" American Economic Review 73(3) pp257-276.

Bernard, Carole and Claudia Czado (2015) "Conditional Quantiles and Tail Dependence" Journal of Multivariate Analysis 138 pp104-126.

Bordo, Michael D. and Joseph G. Haubrich (2008) "The Yield Curve and a Predictor of Grwoth: Long-run Evidence, 1875 - 1997" Review of Economics and Statistics 90(1) pp182-185.

Bordo, Michael D. and Joseph G. Haubrich (2010) "Credit Crises, Money and Contractions: An Historical View" Journal of Monetary Economics 57(1) pp1-18.

Caldara, Dario and Edward Herbst (2019) "Monetary Policy, Real Activity, and Credit spreads: Evidence from Bayesian Proxy SVARs" American Economic Journal: Macroeconomics 11(1) pp157-92.

Carriero, Andrea, Todd E. Clark and Massimiliano Marcellino (2019) "Capturing Macroeconomic Tail Risks with Bayesian Vector Autoregressions" unpublished manuscript.

Charpentier, Arthur, Jean-David Fermanian, and Ollivier Scaillet (2007) The Estimation of Copulas: Theory and Practice, Copulas: from Theory to Applications in Finance.

Chavleishvili, Sulkhan and Simone Manganelli (2019) "Forecasting and Stress Testing with Quantile Vector Autoregression" unpublished manuscript. 
Chen, Song X. (1999) "Beta Kernel Estimators for Density Functions" Computational Statistics and Data Analysis 31(2) pp131-145.

Coe, Patrick J. (2002) "Financial Crisis and the Great Depression: A Regime-Switching Approach" Journal of Money, Credit and Banking 34(1) pp76-93.

Deheuvels, Paul (1979) "La Fonction de Dépendence Empirique et Ses Propriétés. Un Test non Paramétrique d'Indépendence" Bulletin Royal Belge de l'Académie des Sciences 65 pp274-292.

Deheuvels, Paul (1981) "An Asymptotic Decomposition for Multivariate Distribution-free Tests of Independence" Journal of Multivariate Analysis 11(1) pp102-113.

DiNardo, John and Justing L. Tobias (2001) "Nonparametric Density and Regression Estimation" Journal of Economic Perspectives 15(4) pp11-28.

Diebold, Francis X. and Roberto S. Mariano (1995) "Comparing Predictive Accuracy" Journal of Business and Economic Statistics 13(3) pp. 253-63.

Fernández-Villaverde, Jesús and Juan F. Rubio-Ramirez (2004), "Comparing Dynamic Equilibrium Models to Data: a Bayesian Approach" Journal of Econometrics 123(1) pp153-187.

Ferrara, Laurent, M. Mogliani and J-G. Sahuc "Real-time High-frequency Monitoring of Growth-at-Risk" unpublished manuscript.

Friedman, Milton and Anna J. Schwartz (1963) A Monetary History of the United States Princeton: Princeton University Press.

Frydman, Carola, Eric Hilt and Lily Y. Zhou (2015) "Economic Effects of Runs on Early "Shadow Banks": Trust Companies and the Impact of the Panic of 1907" Journal of Political Economy 123(4) pp902-940.

Gertler, Mark, and Peter Karadi (2015) "Monetary Policy Surprises, Credit Costs, and Economic Activity" American Economic Journal: Macroeconomics 7(1) pp44-76.

Harvey, David, Stephen Leybourne, and Paul Newbold (1997) "Testing the Equality of Prediction Mean Squared Errors" International Journal of Forecasting 13(2) pp281-291.

Jalil, Andrew J. (2015) "A New History of Banking Panics in the United States, 1825-1929: Construction and Implications" American Economic Journal: Macroeconomics 7(3) pp295330 . 
Joe, Harry (2006) "Generating Random Correlation Matrices Based on Partial Correlations" Journal of Multivariate Analysis 97(10) pp2177-2189.

Jordà, Òscar, Moritz Schularick and Alan M. Taylor (2013) "When Credit Bites Back" Journal of Money, Credit and Banking 45(2) pp3-28.

Jordà, Òscar, Moritz Schularick, and Alan M. Taylor (2017) "Macrofinancial History and the New Business Cycle Facts" in NBER Macroeconomics Annual 2016 Volume 31, edited by Martin Eichenbaum and Jonathan A. Parker, Chicago: University of Chicago Press.

Karagedikli, Ozer, Shaun P. Vahey and Elizabeth C. Wakerly (2019) "Improved Methods for Combining Point Forecasts for an Asymmetrically Distributed Variable" CAMA Working Paper No. $15 / 2019$.

Karra, Kiran (2018) "Modeling and Analysis of Non-Linear Dependencies using Copulas, with Applications to Machine Learning" PhD thesis, Virginia Polytechnic Institute and State University.

Kascha, Christian and Francesco Ravazzolo (2010) "Combining Inflation Density Forecasts" Journal of Forecasting 29(1-2) pp231-250.

Kullback, S. and R.A. Leibler (1951) "On Information and Sufficiency" Annals of Mathematical Statistics 22(1) pp79-86.

Kullback, S. (1987) "Letter to the Editor: The Kullback-Leibler Distance" The American Statistician 41(4) pp340-341.

Li, Qi and Jeffrey S. Racine (2006) Nonparametric Econometrics: Theory and Practice, Princeton University Press, Princeton.

Loaiza-Maya, Ruben and Michael S. Smith (2019) "Real-Time Macroeconomic Forecasting With a Heteroskedastic Inversion Copula" Journal of Business and Economic Statistics forthcoming.

Loria, Francesca, Christian Matthes, and Donghai Zhang (2019) "Assessing Macroeconomic Tail Risk" unpublished manuscript.

Macaulay, Frederick R. (1938) The Movement of Interest Rates, Bond Yields and Stock Prices in the United States Since 1856 NBER.

Nelsen, Roger B. (2006) An Introduction to Copulas, 2nd ed., New York: NY: Springer. 
Robertson, John. C., Ellis W. Tallman and Charles H. Whiteman (2005) "Forecasting Using Relative Entropy" Journal of Money, Credit and Banking 37(3) pp383-401.

Rockoff, Hugh (1990) "The Wizard of Oz as a Monetary Allegory" Journal of Political Economy 98(4) pp739-760.

Romer, Christina D. (1989) "The Prewar Business Cycle Reconsidered: New Estimates of Gross National Product, 1869-1908" Journal of Political Economy 97(1) pp1-37.

Schularick, Moritz and Alan M. Taylor (2012) "Credit Booms Gone Bust: Monetary Policy, Leverage Cycles and Financial Crises, 1870 - 2008" American Economic Review 102(2) pp1029-1061.

Shiller, Robert J. (2000) Irrational Exhuberence, Princeton University Press, Princeton.

Shimazaki, Hideaki and Shigeru Shinomoto (2010) "Kernel Bandwidth Optimization in Spike Rate Estimation" Journal of Computational Neuroscience 29(1-2) pp171-182.

Scott, David. W. (1992) Multivariate Density Estimation: Theory, Practice, and Visualization, John Wiley and Sons, New York.

Silverman, Bernard. W. (1986) Density Estimation for Statistics and Data Analysis, Chapman and Hall, New York.

Smith, Michael S. (2015) "Copula Modelling of Dependence in Multivariate Time Series" International Journal of Forecasting 31(3) pp815-833.

Smith, Michael S. and Shaun P. Vahey (2016) "Asymmetric Forecast Densities for U.S. Macroeconomic Variables from a Gaussian Copula Model of Cross-Sectional and Serial Dependence" Journal of Business and Economic Statistics 34(3) pp416-34.

Velásquez-Giraldo, Mateo, Gustavo Canavire Bacarreza, Kim P. Huynh and David T. JachoChavez (2018) "Flexible Estimation of Demand Systems: A Copula Approach" Journal of Applied Econometrics 33(7) pp1109-1116. 
Table 1: Summary Statistics - Output Growth

\begin{tabular}{lcccc}
\hline & $1875: 3-1913: 4$ & $1919: 2-1941: 3$ & $1946: 2-1971: 2$ & $1971: 4-2017: 4$ \\
\cline { 2 - 5 } Annualized 1 Quarter Growth Rate & & & \\
Mean & 4.08 & 2.75 & 3.31 & 2.77 \\
Standard Deviation & 8.43 & 12.5 & 4.47 & 3.22 \\
Skewness & -0.37 & -0.41 & -0.51 & -0.53 \\
Kurtosis & 5.34 & 2.70 & 4.61 & 5.67 \\
Shapiro-Wilk p-value & $<0.001$ & 0.133 & 0.011 & $<0.001$ \\
& & & & 185 \\
Number of Observations & 154 & 90 & 101 & \\
& & & & \\
& $1877: 1-1913: 4$ & $1920: 1-1941: 3$ & $1947: 1-1971: 2$ & $1972: 3-2017: 4$ \\
\cline { 2 - 5 } 4uarter Growth Rate & & & & \\
Mean & 4.26 & 2.81 & 3.51 & 2.80 \\
Standard Deviation & 6.02 & 9.07 & 3.12 & 2.22 \\
Skewness & -0.23 & -0.40 & 0.05 & -0.52 \\
Kurtosis & 3.59 & 2.21 & 3.23 & 4.00 \\
Shapiro-Wilk $p$-value & 0.031 & 0.009 & 0.728 & $<0.001$ \\
Number of Observations & & & & 182 \\
\hline
\end{tabular}

Notes: 1 quarter growth rates are reported at an annualized rate. The Shapiro-Wilk $p$-value refers to a test of the null hypothesis that the the variable is Gaussian distributed. 
Table 2: Summary Statistics - Financial Variables

\begin{tabular}{|c|c|c|c|c|}
\hline & 1875:1-1913:4 & 1919:1-1945:3 & 1946:1-1971:2 & 1971:3-2017:4 \\
\hline \multicolumn{5}{|l|}{ Term Spread } \\
\hline Mean & -0.27 & 3.22 & 1.38 & 1.72 \\
\hline Standard Deviation & 1.23 & 1.85 & 0.80 & 1.27 \\
\hline Skewness & -0.21 & 0.20 & 0.38 & -0.71 \\
\hline Kurtosis & 3.26 & 2.33 & 4.05 & 3.41 \\
\hline Shapiro-Wilk $p$-value & 0.424 & 0.089 & 0.022 & $<0.001$ \\
\hline \multicolumn{5}{|l|}{ Credit Spread } \\
\hline Mean & 0.81 & 1.99 & 0.66 & 1.09 \\
\hline Standard Deviation & 0.32 & 0.86 & 0.20 & 0.46 \\
\hline Skewness & 0.56 & 1.64 & 1.42 & 1.82 \\
\hline Kurtosis & 2.88 & 6.89 & 6.31 & 7.57 \\
\hline Shapiro-Wilk $p$-value & $<0.001$ & $<0.001$ & $<0.001$ & $<0.001$ \\
\hline \multicolumn{5}{|l|}{ SEP Volatility } \\
\hline Mean & 2.42 & 4.20 & 2.54 & 2.64 \\
\hline Standard Deviation & 1.48 & 3.60 & 1.40 & 1.69 \\
\hline Skewness & 1.45 & 2.71 & 0.87 & 1.04 \\
\hline Kurtosis & 6.21 & 14.1 & 3.92 & 4.26 \\
\hline Shapiro-Wilk $p$-value & $<0.001$ & $<0.001$ & 0.001 & $<0.001$ \\
\hline \multicolumn{5}{|c|}{ National Financial Conditions Index } \\
\hline Mean & & & & 0.013 \\
\hline Standard Deviation & & & & 0.99 \\
\hline Skewness & & & & 1.90 \\
\hline Kurtosis & & & & 6.15 \\
\hline Shapiro-Wilk $p$-value & & & & $<0.001$ \\
\hline Number of Observations & 156 & 91 & 102 & 186 \\
\hline
\end{tabular}

Note: The Shapiro-Wilk $p$-value refers to a test of the null hypothesis that the the variable is Gaussian distributed. 


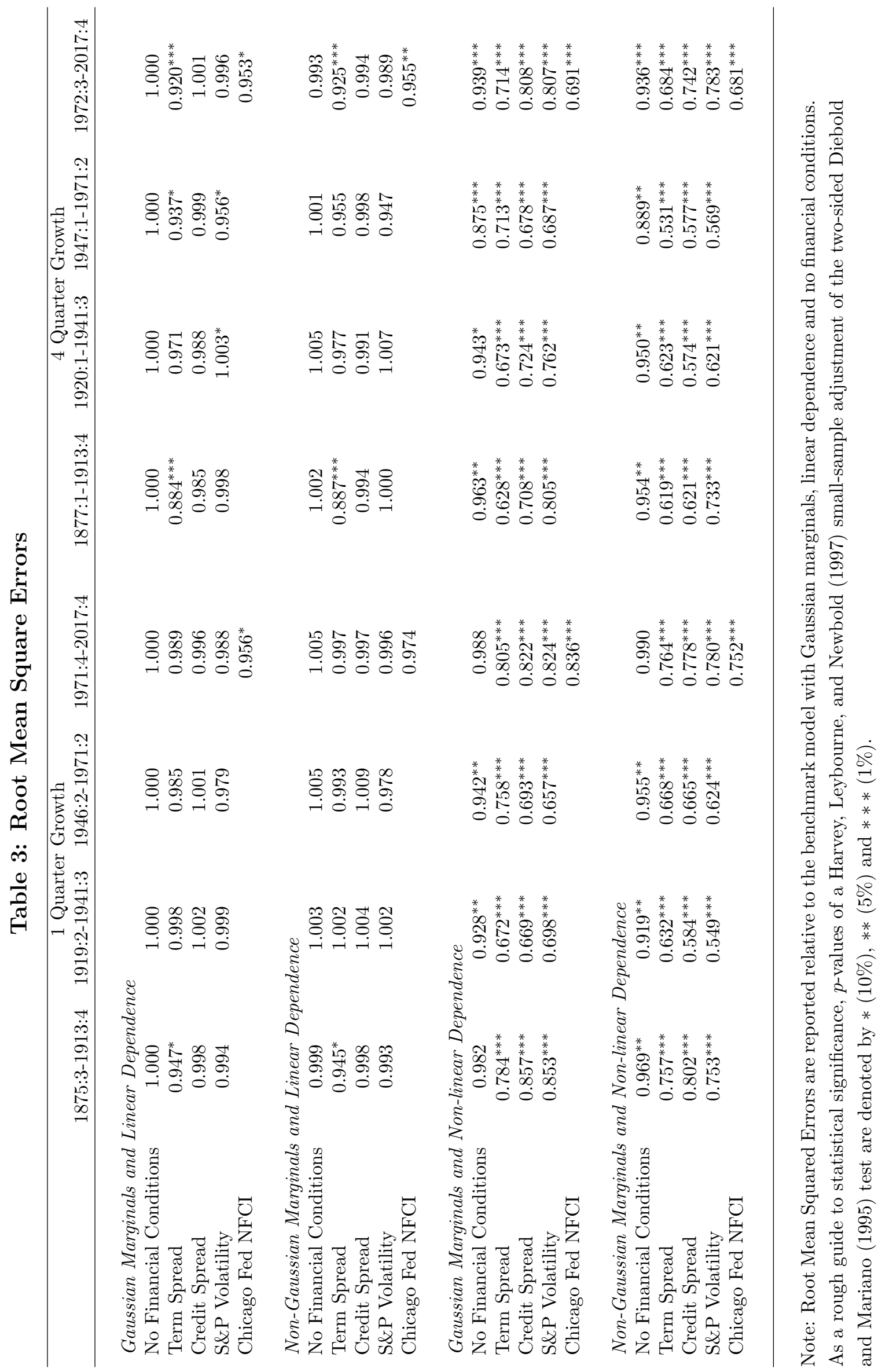




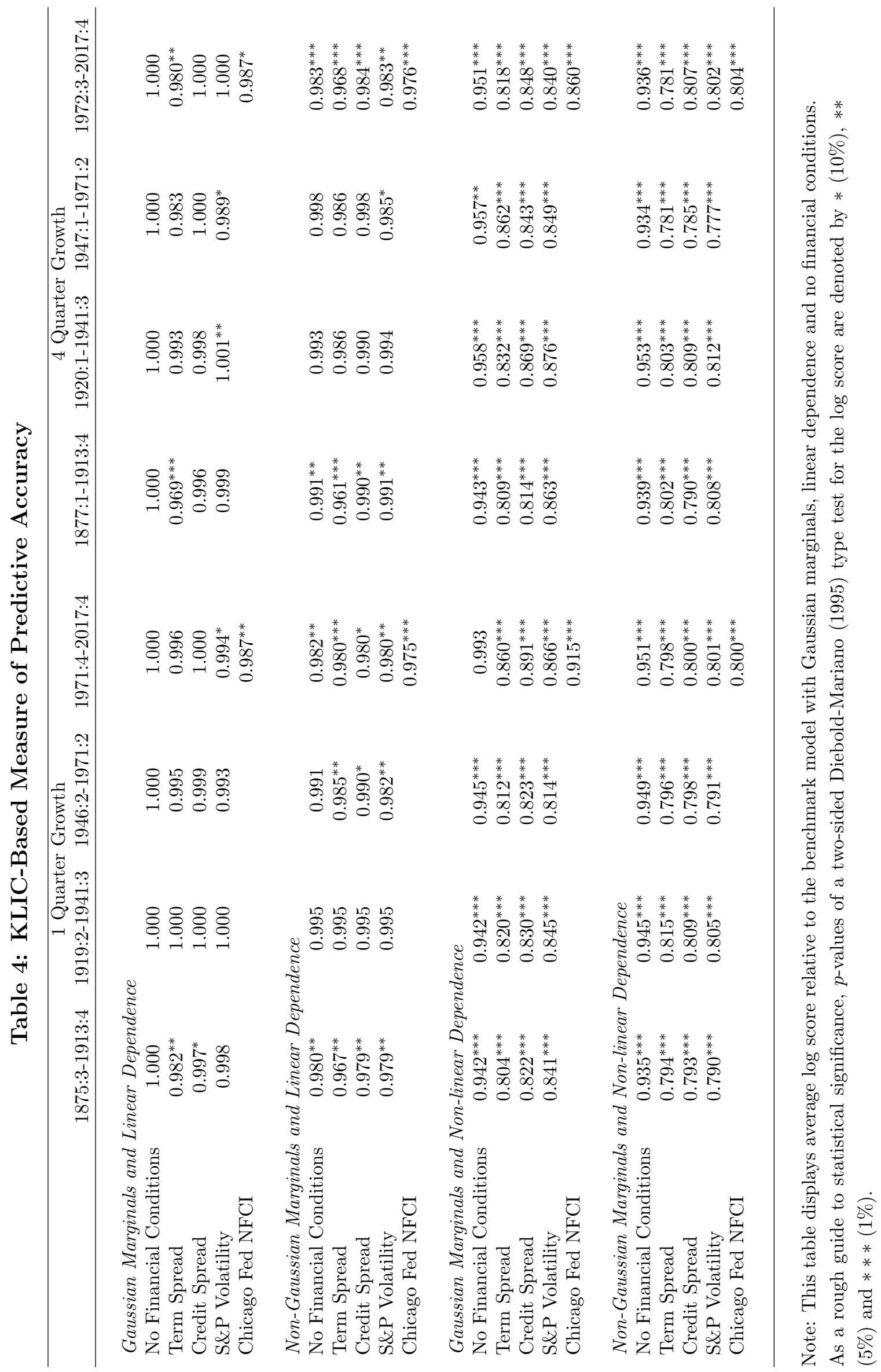


Figure 1: Fitted Marginal Distributions for Output Growth
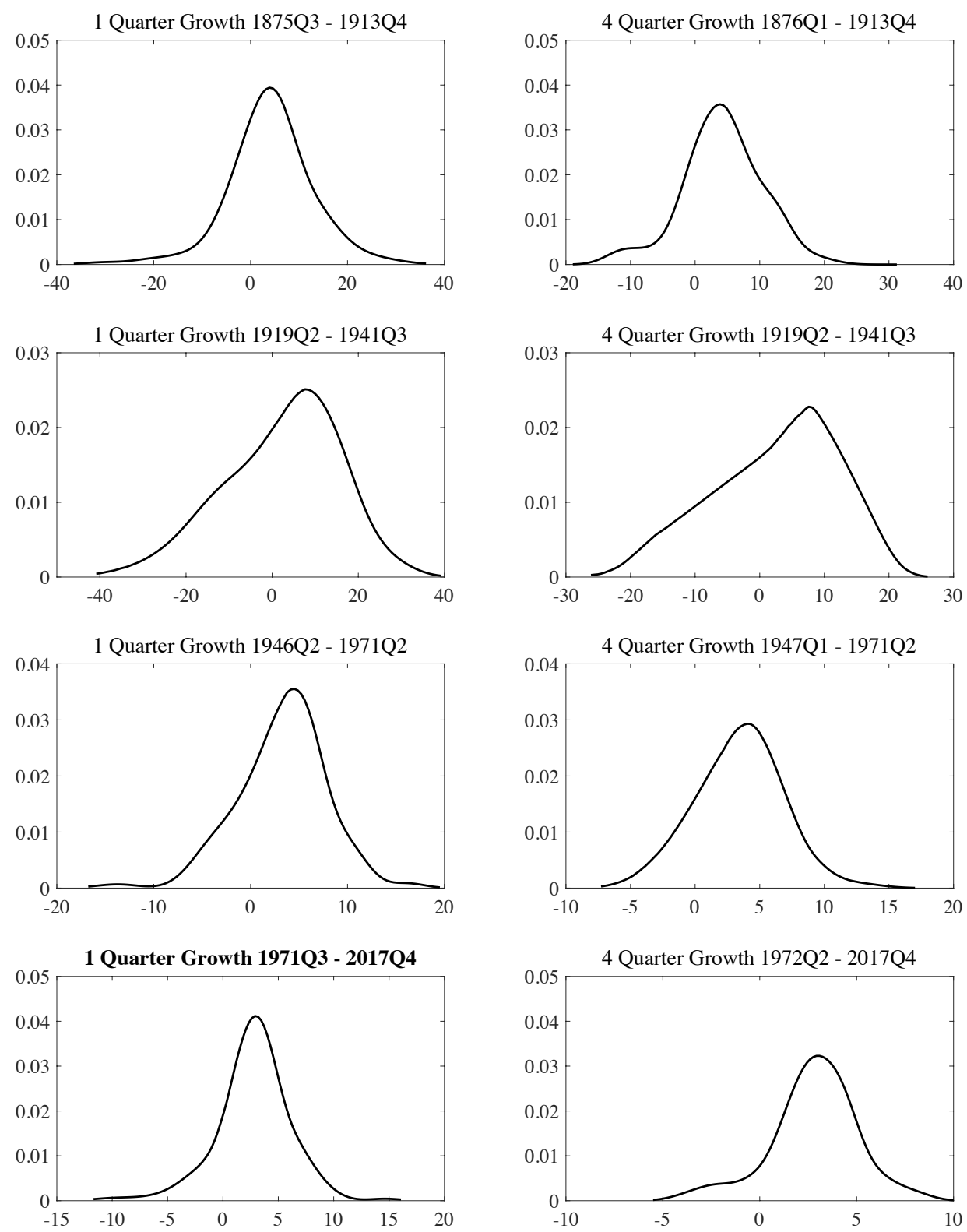

Note: The panels depict the marginal distribution for output growth in each era, fitted using the SSV method of Shimazaki and Shinomoto (2010) and plotted as PDFs. 


\section{Figure 2: Fitted Marginal Distributions for Financial Variables}
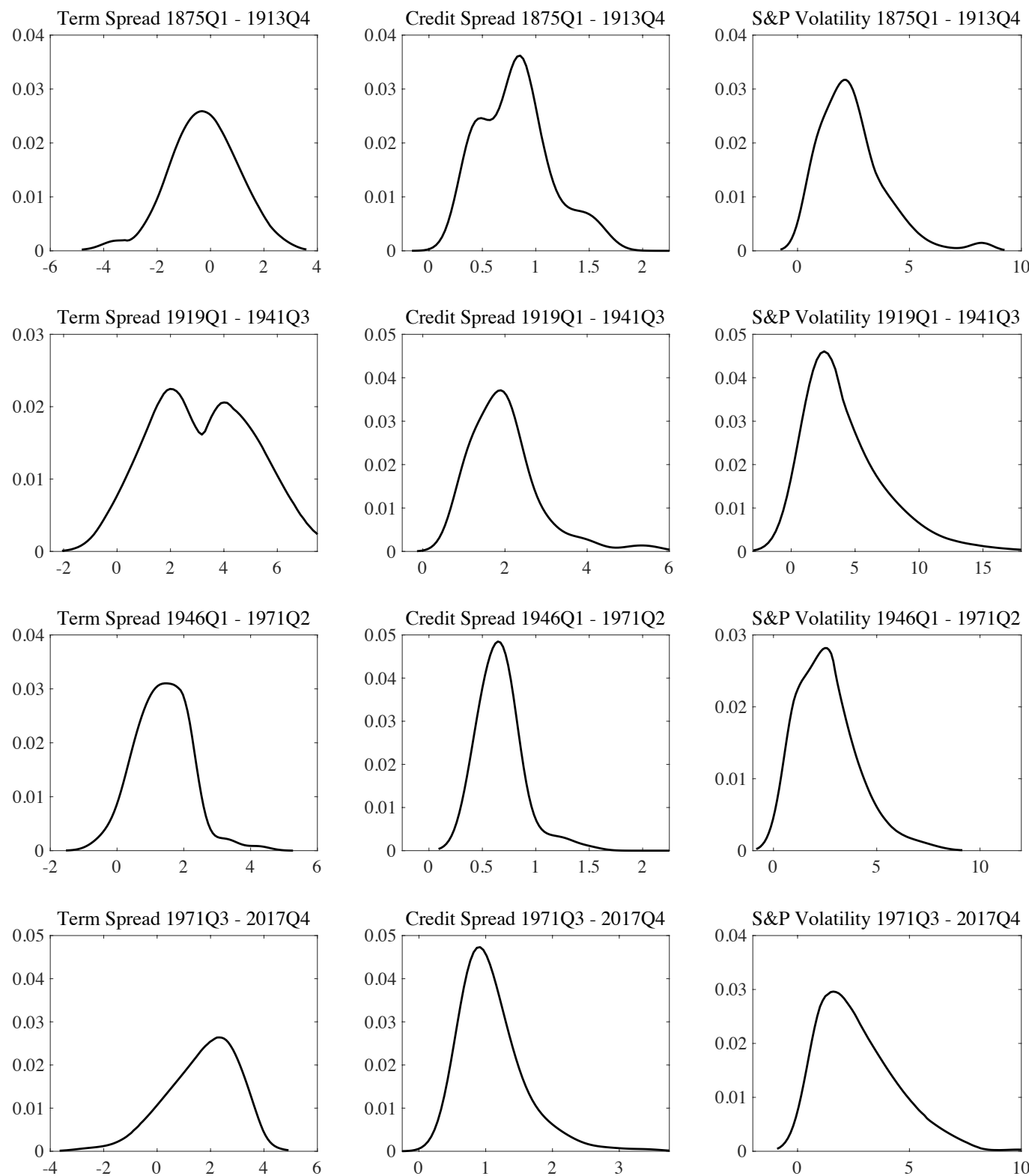

Note: The panels depict the marginal distribution for the term spread, the credit spread and a measure of stock market volatility in each era, fitted using the SSV method of Shimazaki and Shinomoto (2010) and plotted as PDFs. 
Figure 3a: Conditional Densities 1875 - 1913
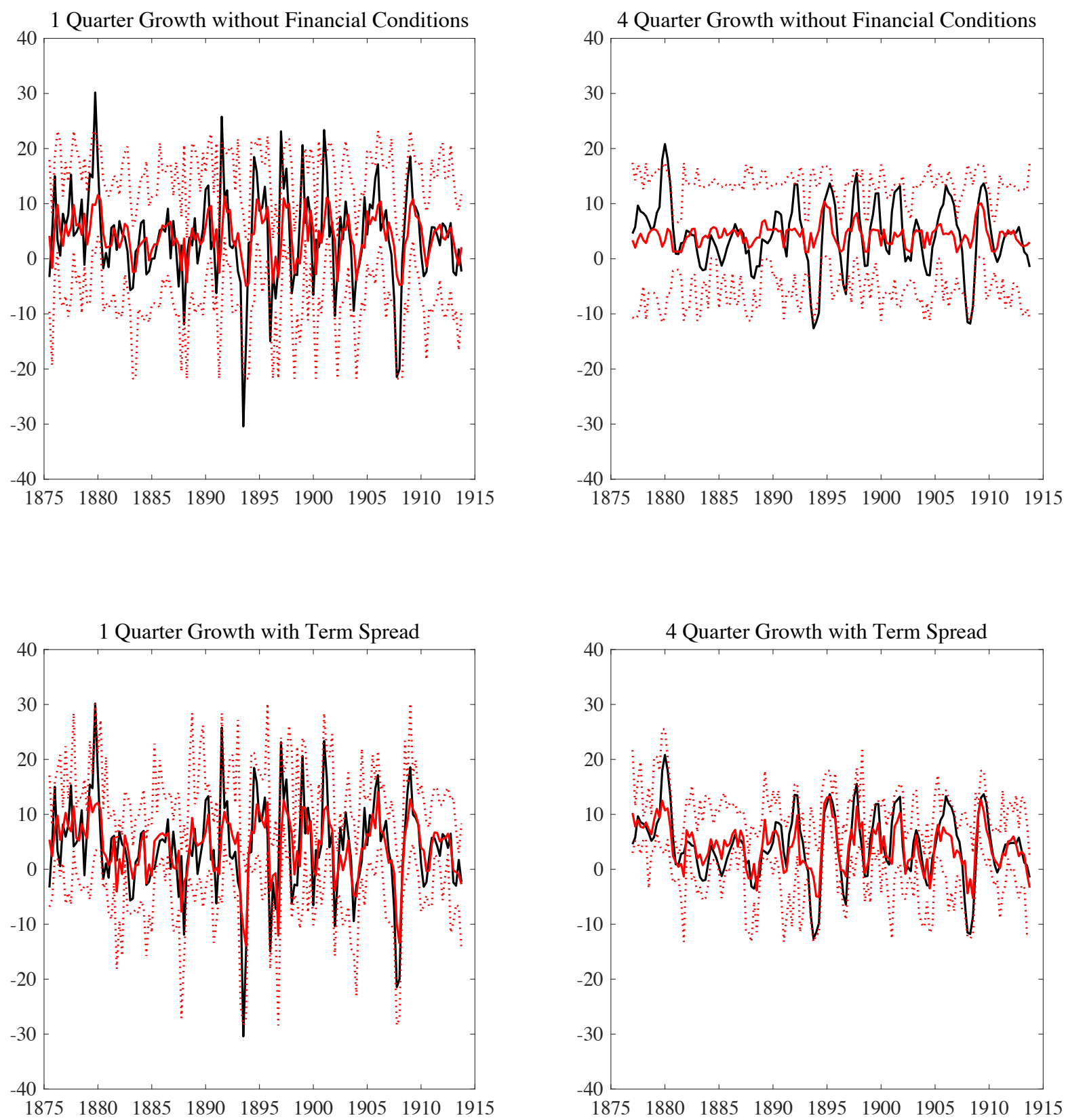

Note: In each panel, the solid line black line depicts the realizations and the solid red line depicts the mean of the conditional density for output growth based on a model with non-Gaussian marginals and non-linear dependence. The dotted red lines depict the $5^{\text {th }}$ and $95^{\text {th }}$ percentiles. 
Figure 3b: Conditional Densities 1919 - 1941
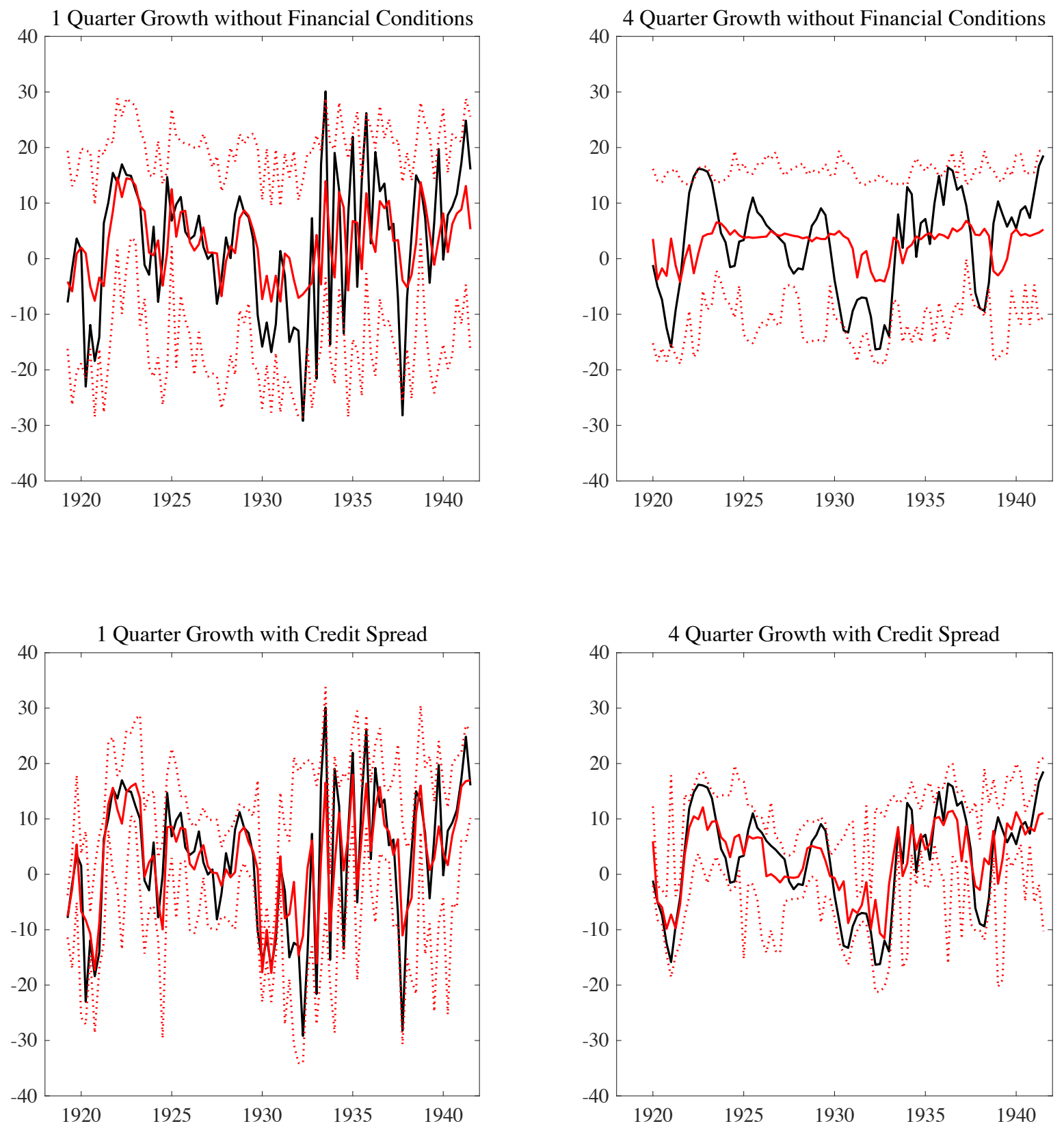

Note: See note to figure 3a. 
Figure 3c: Conditional Densities 1946 - 1971
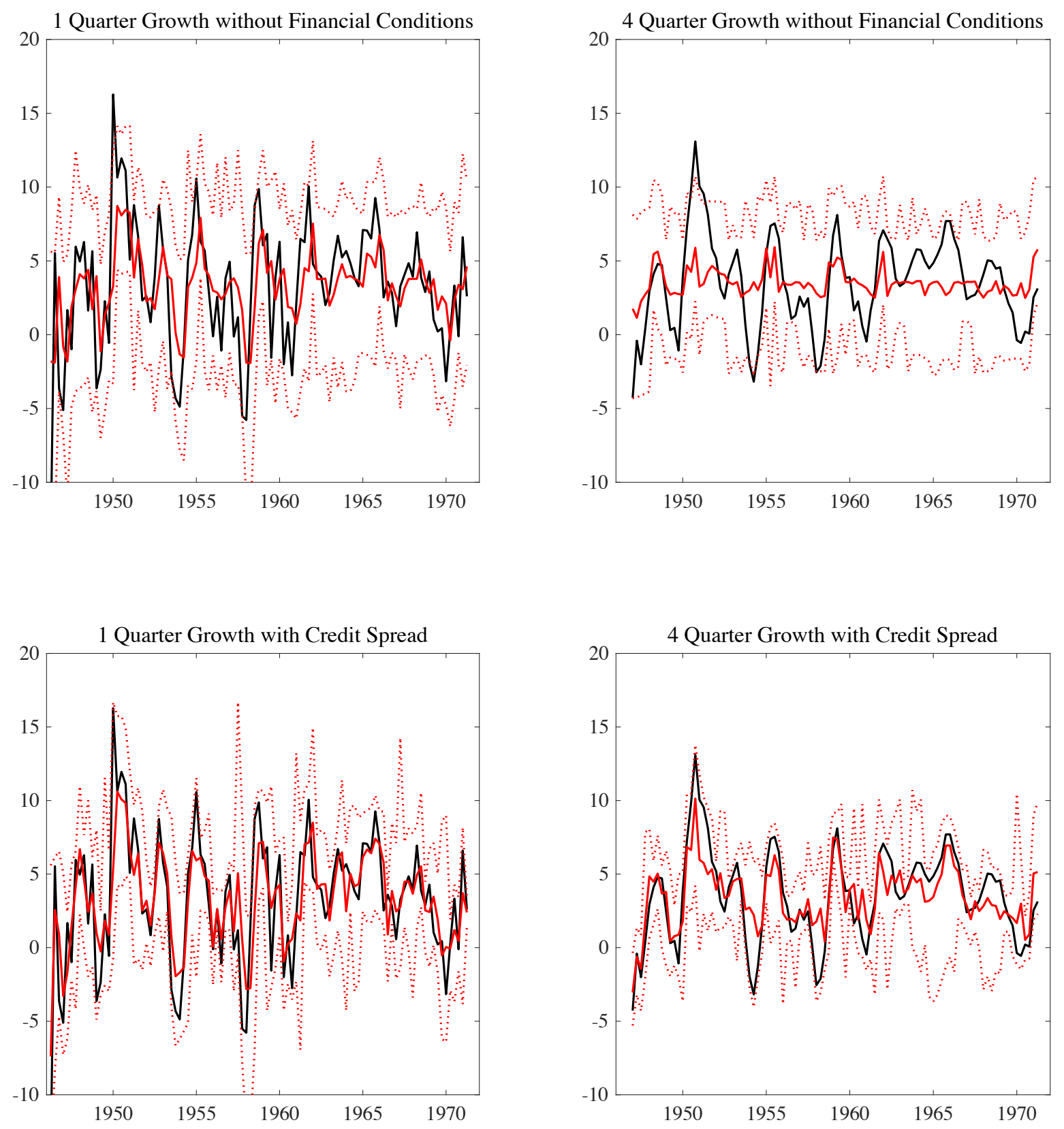

Note: See note to figure 3a. 
Figure 3d: Conditional Densities 1971 - 2017
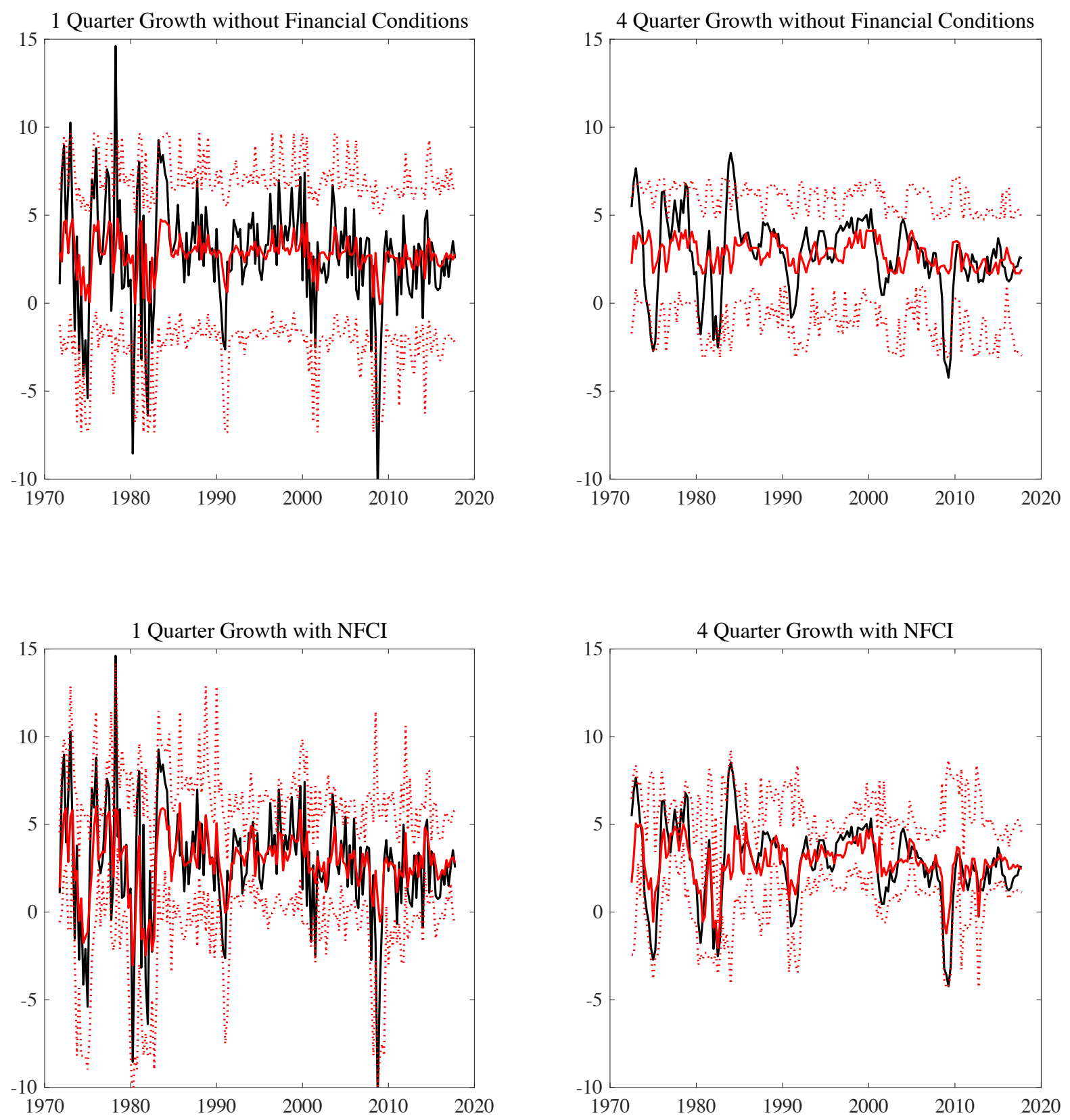

Note: See note to figure 3a. 
Figure 4a: Probability of Tail Events 1875 - 1913
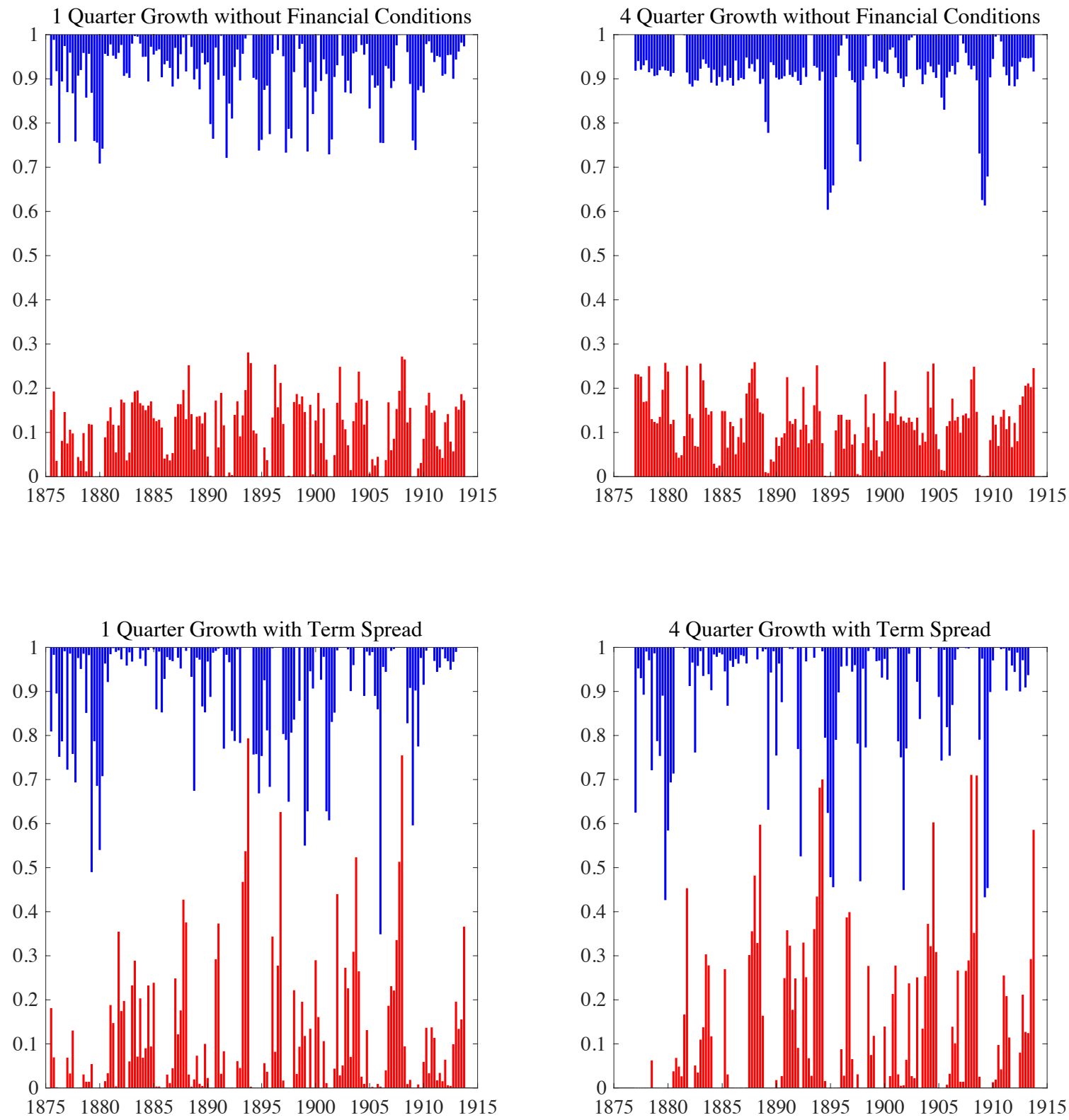

Note: The red bars depict the conditional probability assigned to low output growth events below the $10^{\text {th }}$ percentile of the unconditional distribution for the sub-sample and are measured upwards from zero. The blue bars depict the conditional probability of high output growth events greater than the $90^{\text {th }}$ percentile of the unconditional distribution for the relevant sub-sample and are measured downwards from one. All specifications use non-Gaussian marginals and non-linear dependence. 
Figure 4b: Probability of Tail Events 1919 - 1941
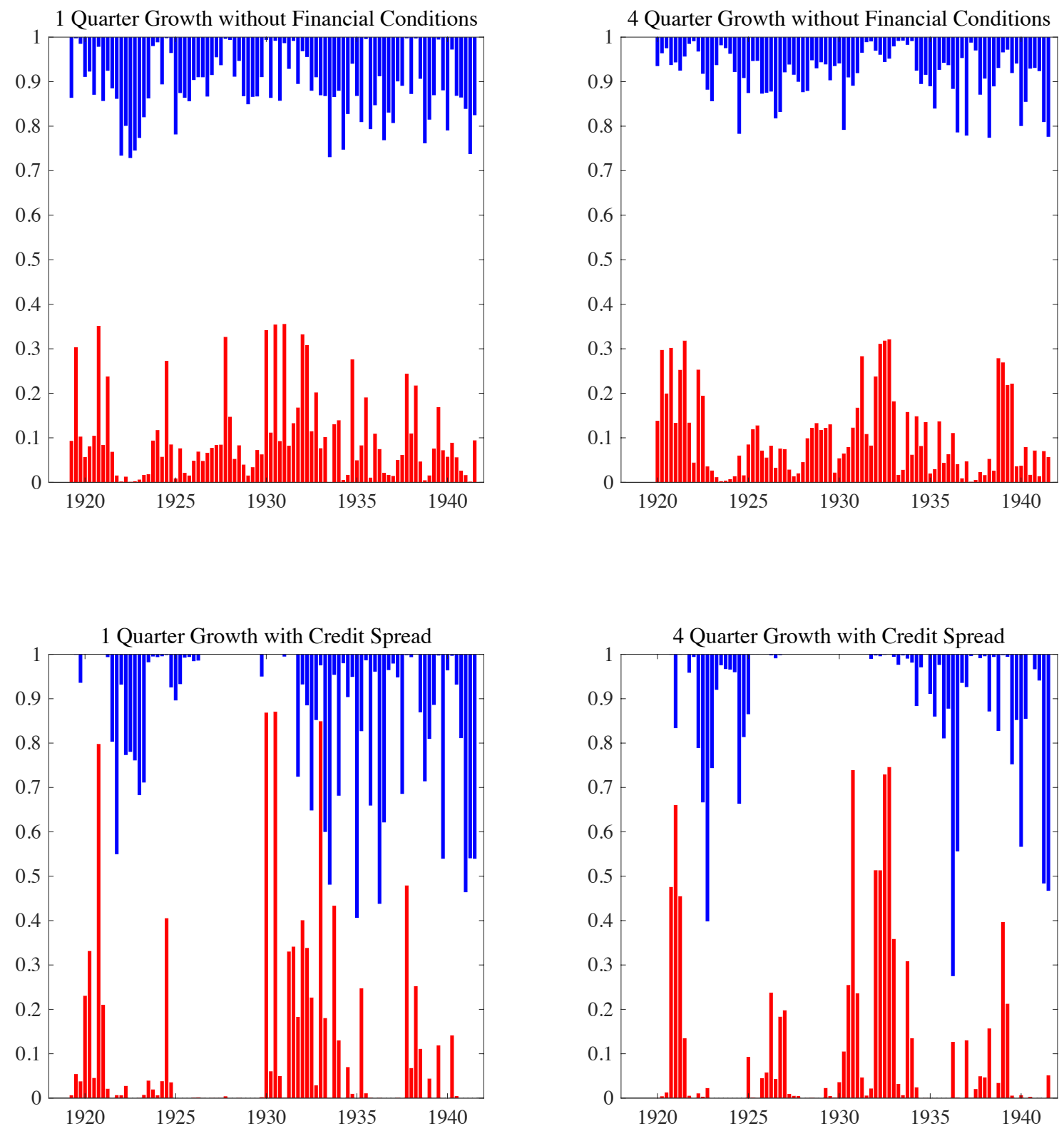

See note to Figure 4a. 
Figure 4c: Probability of Tail Events 1946 - 1971
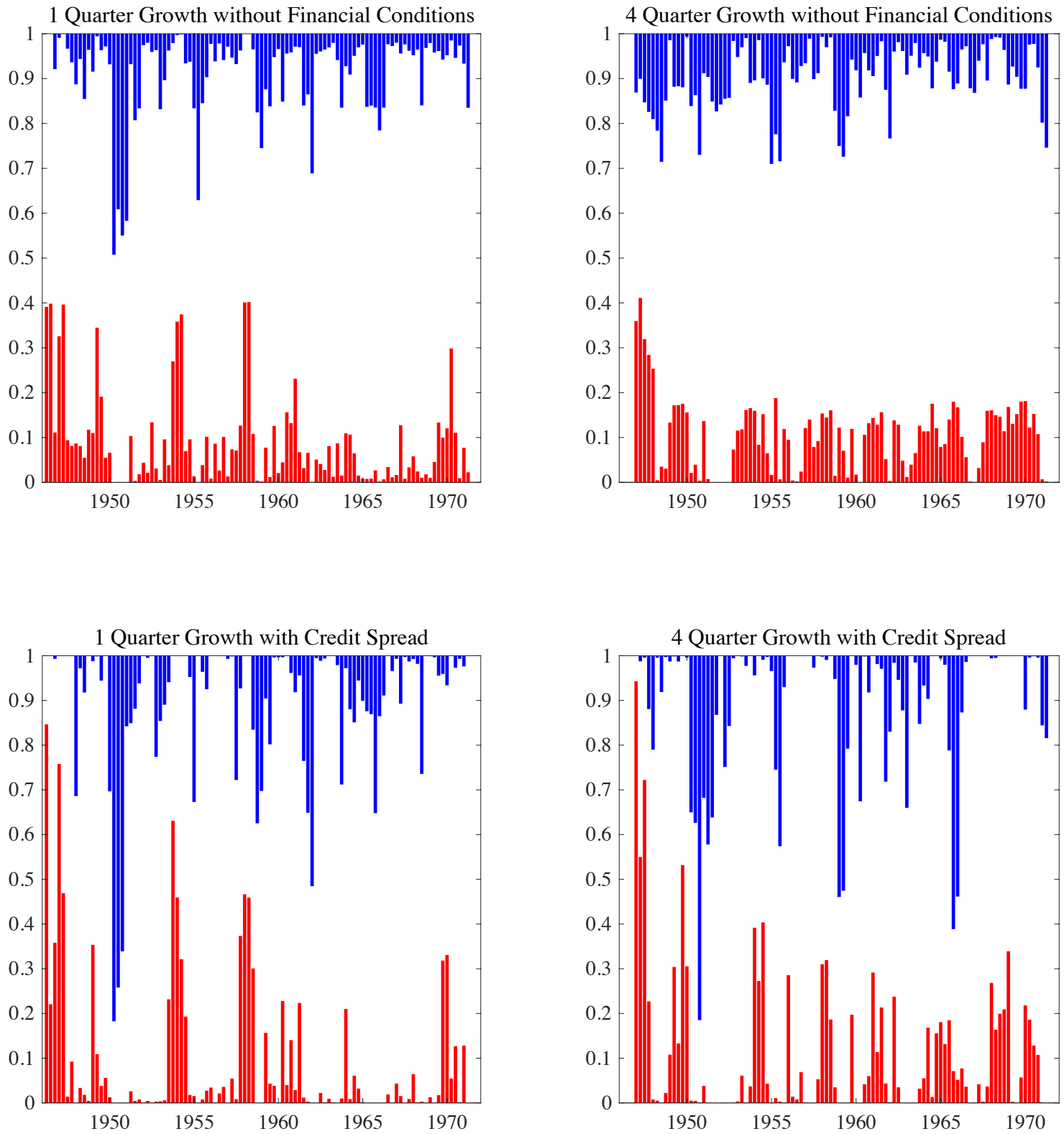

See note to Figure 4a. 
Figure 4d: Probability of Tail Events $1971-2017$
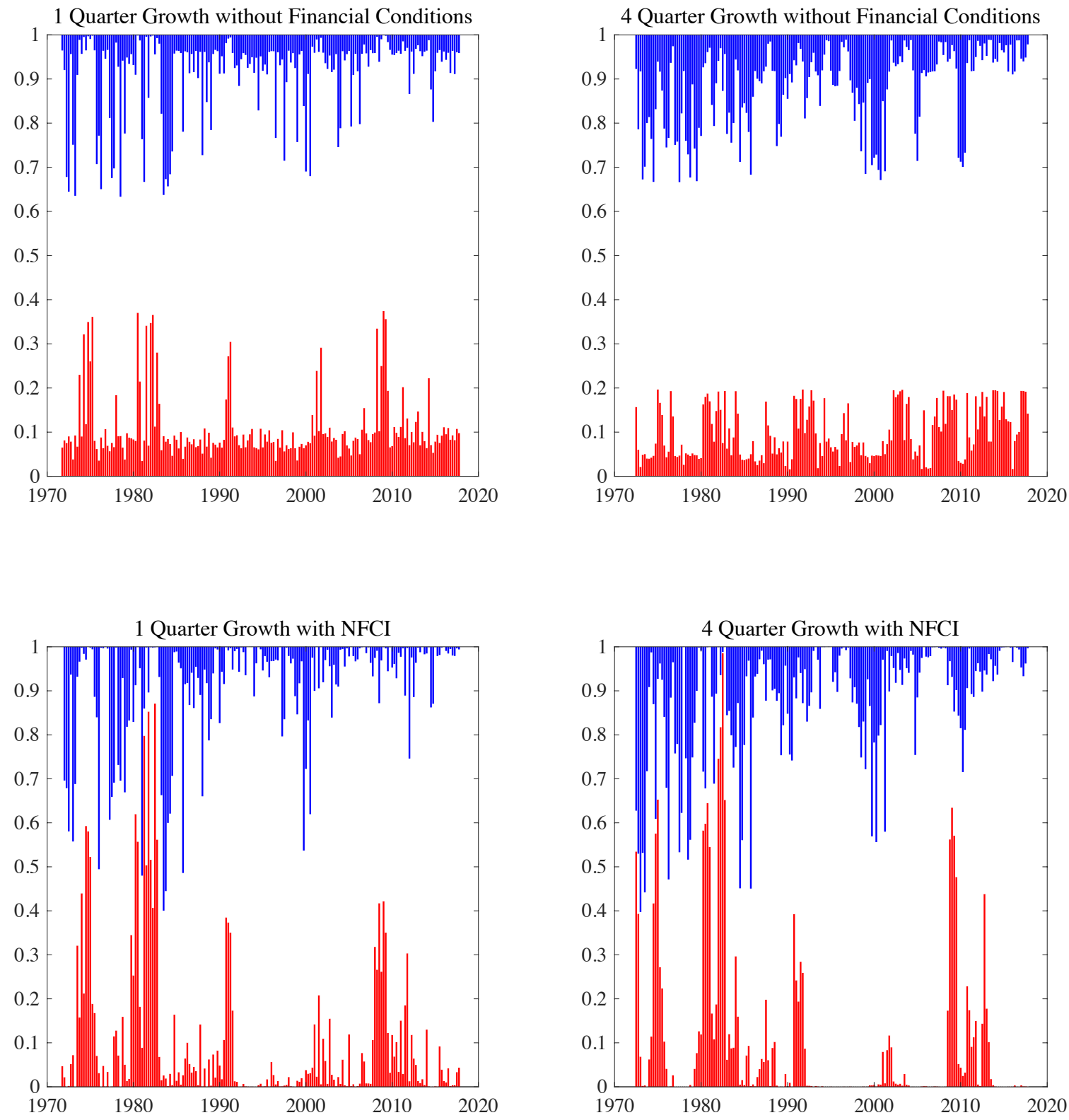

See note to Figure 4a. 
Figure 5: Predictive Densities For Selected Financial Crises
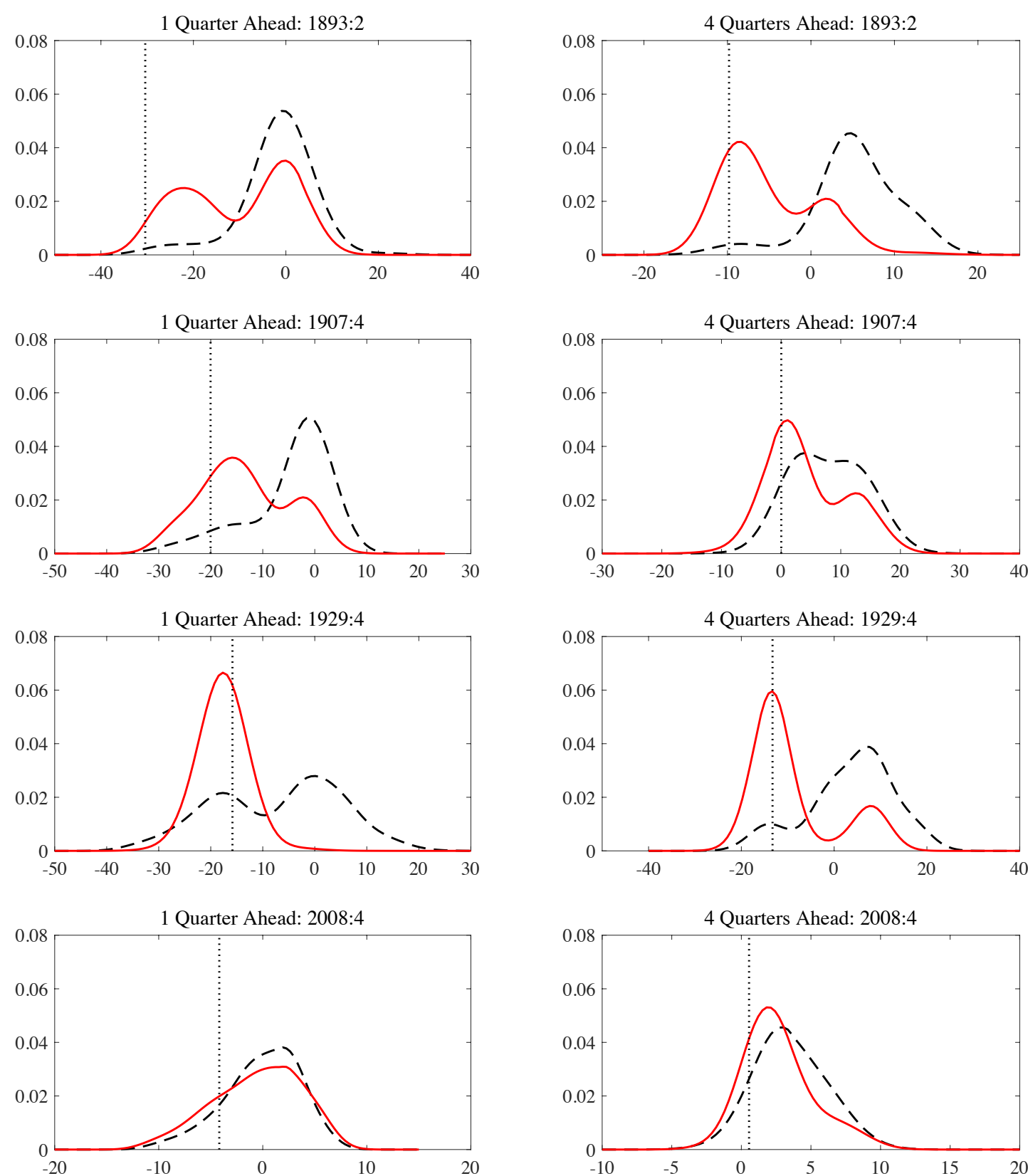

Note: The dashed black line depicts the conditional density based on the specification with non-Gaussian marginals and non-linear dependence, without financial conditions. The solid red line depicts the equivalent density accounting for financial conditions. The measures of financial conditions are the term spread (first two rows), the credit spread (third row) and the NFCI (fourth row). The left (right) panels display the densities for output growth in the subsequent quarter (year). 


\title{
Appendix to Financial Conditions and the Risks to Economic Growth in the United States Since 1875
}

\author{
Patrick J. Coe \\ Shaun P. Vahey \\ Carleton University \\ University of Warwick \\ and CAMA (ANU) \\ and CAMA (ANU)
}

April 3, 2020

\begin{abstract}
This not for publication appendix contains further details on the data and some additional results.
\end{abstract}

\section{Contents}

1 Data Sources and Construction $\quad$ A.2

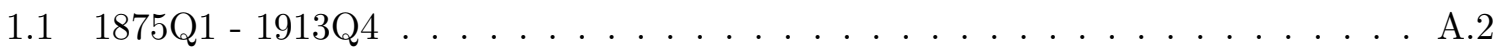

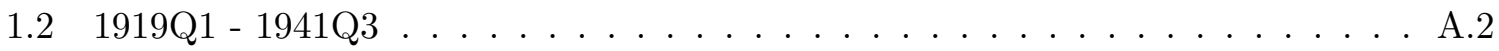

$1.2 .11914 \mathrm{Q} 1-1945 \mathrm{Q} 4 \ldots \ldots \ldots \ldots \ldots \ldots \ldots$

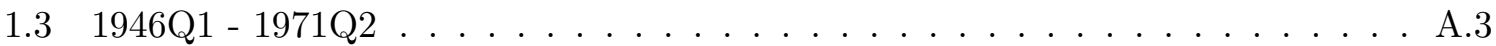

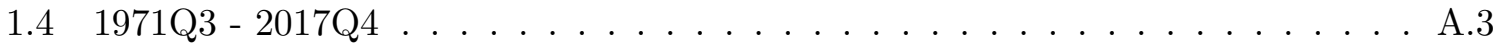

2 Bandwidth Selection in Copula Estimation $\quad$ A.4

$\begin{array}{lll}3 & \text { Recursive RMSE and KLIC } & \text { A.4 }\end{array}$

4 Additional Results $\quad$ A.4

4.1 Alternative Interwar Period 1914Q1 to 1945Q4 . . . . . . . . . . . . A.4

4.2 Common Dependence Across Eras . . . . . . . . . . . . . . . . . A.5

4.3 Plots for Alternative Financial Variables . . . . . . . . . . . . . . . A.5

4.4 Probability Plots for Alternative Definitions of Low and High Growth . . . . . A.6 


\section{Data Sources and Construction}

The quarterly data used in this paper comes from the FRED and NBER Macrohistory database, Balke Gordon (1986), and Shiller (2000). Shiller continues reporting data beyond the publication date of his book on his website. See http://www.econ.yale.edu/ shiller/data.htm. For output growth, the term spread and the credit spread, no single source provides a series that spans the whole of 1875 - 2017. Below we outline the variables and our data sources for each of our four sub-samples, and an alternative sub-sample for our second era. We also outline the construction of our quarterly stock market volatility variable from Shiller's S\&P index. Figures A1a to A1e present plots of the data.

\section{$1.1 \quad 1875 \mathrm{Q} 1-1913 \mathrm{Q} 4$}

To measure output growth we use annualized one quarter and four quarter growth rates of real GNP. For this sub-sample we use the real GNP series from Balke and Gordon. The data reported in Balke and Gordon is seasonally unadjusted and so we first seasonally adjust in Matlab using a seasonal filter as outlined at https://www.mathworks.com/help/econ/seasonaladjustment-using-snxd7m-seasonal-filters.html For the term spread we use data collected from the NBER Macrohistory database. Our series is very similar to the one constructed by Balke and Gordon, and only differs in the fact that we use the last month of each quarter as the observation for that quarter, rather than the first month. This difference is continued in the construction of all quarterly term and credit spread series used in the paper.

First we construct a quarterly Corporate Bond Yield to represent the long-term interest rate using Macaulay's (1938) Railroad Bond Yields Index which is based on all bonds having at least 10 years maturity (NBER Macrohistory database series M13019a). We then use the New York City Commercial Paper Rate from the NBER Macrohistory database (series M13002) to represent the short term interest rate. This is a 60-90 day rate from Macaulay (1938). Subtracting the Commercial Paper Rate from the Corporate Bond Yield gives a term spread. We also use data from the NBER Macrohistory database to construct a credit spread as the Yield on Railroad Bonds minus the Yield on High Grade Railway Bonds. Both of these series are originally from Macaulay (1938) and in the NBER Macrohistory database are M13019a and M13019 respectively. Finally, we use Shiller's S\&P Composite Index to create a measure of stock market volatility. Using Shiller's monthly series we calculate the growth rate of the S\&P index for each month as:

$$
\Delta S P I_{t}=100\left(\frac{S P I_{t}}{S P I_{t-1}}-1\right)
$$

Then, for each quarter, we measure stock market volatility as the standard deviation of $\Delta S P I_{t}$ over the three months of that quarter.

\section{$1.2 \quad 1919 Q 1-1941 Q 3$}

For output growth and the measure of stock market volatility we use the same sources that we use for our 1875Q1 - 1913Q4 sample. For the term spread we subtract the NBER Macrohistory database's New York City Commercial Paper Rate from a long-term corporate bond yield. As mentioned before, the Commercial Paper Rate reported in the NBER Macrohistory database is from Macaulay for 1875 to 1936. It is then computed from weekly data by the NBER from 1937 to 1942, and then taken from the Federal Reserve Board from 1943 to 1971. We follow 
Balke and Gordon (1986) for the choice of long-term corporate bond yield and use the yield on Moody's Baa rated bonds which we obtain from the FRED database (series BAA). For the credit spread we use the difference between the yields on Moody's Baa rated bonds and Moody's Aaa rated bonds (series AAA), both of which are taken from the FRED database.

\subsubsection{Q1 - 1945Q4}

In this appendix we also report results using alternative timings for our second sub-sample to include both world wars. That is, 1914Q1 to 1945Q4. The source for the output growth data and our measure of stock market volatility are the same as for the 1919Q1 to 1941Q3 sub-sample. For the term spread we follow Balke and Gordon (1986) and use the Yield on Railway Bonds (until 1918Q4) and the Moody's Baa Yield (from 1919Q1) to represent the Long Term Corporate Bond Yield, and subtract the New York City Commercial Paper Rate from this.

We combine data from the NBER Macrohistory and FRED databases to construct a credit spread. The spread based on railway bond data can be extended to 1934Q4 and therefore overlaps with the Baa - Aaa spread from 1919Q1 to 1934Q4. For this period of overlap the estimated correlation between the two series is 0.90. Figure A2 plots these two series for overlap. To create a single series for the credit spread we first scale the credit spread based on the Railway Bond Yields $\left(X_{t}\right)$ for the period 1875Q1 to 1918Q4 according to:

$$
x_{t}=\frac{X_{t}-\mu_{\text {Railway }}}{\sigma_{\text {Railway }}}
$$

where $\mu_{\text {Railway }}=0.4763$ and $\sigma_{\text {Railway }}=0.3387$ are the mean and standard deviation of $X_{t}$ for the period 1919Q1 to 1934Q4. We then scale $x_{t}$ according to:

$$
z_{t}=x_{t} * \sigma_{M o o d y^{\prime} s}+\mu_{M o o d y^{\prime} s}
$$

where $\mu_{\text {Moody's }}=2.0050$ and $\sigma_{M o o d y^{\prime} s}=0.9911$ are the mean and standard deviation of the Baa - Aaa spread for 1919Q1 to 1934Q4. Finally, a single series is constructed using $z_{t}$ for 1914Q1 to 1918Q4 and the Baa - Aaa spread from 1919Q1 onwards.

\section{$1.3 \quad 1946 \mathrm{Q} 1$ - 1971Q2}

For 1946Q1 to 1971Q2 we use the same sources as for 1919Q1 to 1941Q3 for all variables.

\subsection{Q3 - 2017Q4}

To measure output growth we use annualized one quarter and four quarter growth rates of real GNP constructed using data from the FRED database (series GNPC96). To construct the term spread we now use data from the FRED database. To be specific, we subtract the 3-Month Treasury Bill, Secondary Market Rate (series TB3MS) from the 10-Year Treasury Constant Maturity Rate (series GS10). For the credit spread and the measure of stock market volatility we use the same sources as for 1946Q1 to 1971Q2. We also use the Chicago Federal Reserve Bank's National Financial Conditions Index as a measure of financial conditions, taken from the FRED database (series NFCI). 


\section{Bandwidth Selection in Copula Estimation}

When estimating our copula specifications with non-linear dependence we use a value of $h=$ 0.01 as the kernel bandwidth used to smooth over $K=500$ gridpoints. In this section we discuss bandwidth choice. Selecting a bandwidth that is too high can mean that the resulting density is over smoothed and so features of the underlying dependence between variables are hidden. On the other hand a value of $h$ that is too low can lead to under smoothing and an estimated density that reflects noise in the data rather than the underlying dependence structure.

To explore this issue we performed an out of sample forecasting exercise based on our copula specification that uses ECDF marginals and allows for non-linear dependence. In this exercise we use data from our fourth subsample, namely 1971:3 to 2017:4. We begin by estimating the copula without financial conditions using data from 1971:3 to 1990:1 and then we use these estimates to predict output growth in 1990:2. We then expand the estimation sample one quarter, and estimate using data from 1971:2 to 1990:2 and use these new estimates to forecast 1990:3. We continue this recursive process to obtain out of sample forecasts for 1990:2 to $2017: 4$.

We repeat this exercise using a number of different values for $h$ in our copula estimation. Table A1 presents root mean squared errors and KLIC based measures of predictive accuracy for out of sample forecasts for various bandwidth choices. For each alternative bandwidth these statistics are reported relative to the bandwidth choice of $h=0.01$ that we employ in the main text. For the values of $h$ from 0.001 to 1.28 we see very little difference in these measures of out of sample fit relative to our chosen value of $h=0.01$. It is not until we lower the bandwidth to $h=0.0001$ that we see the significant worsening of out of sample forecasting performance that we would associate with in sample overfitting. In addition, we do not see any significant improvement in fit when we use values of $h$ above $h=0.01$.

\section{Recursive RMSE and KLIC}

In the main text we report RMSE and KLIC based measures of fit which indicate that the addition of a measure of financial conditions greatly improves in-sample fit in our preferred specification with ECDF marginals and non-linear dependence. In this appendix we plot recursively calculated RMSE and KLIC based measures in Figures 3a to 3d. We do this for our baseline specification which uses Gaussian marginals and linear dependence, but not financial conditions, and compare this with our preferred specification which uses ECDF marginals and non-linear dependence. In this exercise we consider our preferred specification with and without financial conditions. These plots show that the recursive measures of fit are superior for our preferred specification with financial conditions throughout the various sub-samples. Furthermore, changes in relative performance across specifications tend to be relatively small and gradual, consistent with performance gains being robust to outliers.

\section{Additional Results}

\subsection{Alternative Interwar Period 1914Q1 to 1945Q4}

In this appendix we report results for an alternative second sub-sample in which the years of the first and second world wars are included. Table A2 reports summary statistics for 
our output growth and financial conditions measures for 1914:1 to 1945:4. A comparison of the summary statistics for output growth in this table with those reported for 1919:1 to 1941:3 in the main text indicates that the inclusion of the data from the war years raises the mean growth rates by about one percentage points, but that the standard deviation, skewness and kurtosis are largely unchanged. The Shapiro-Wilk statistics continue to point to a rejection of normality. Similarly, the statistics for the measures of financial conditions are largely unchanged. Nevertheless, there is less evidence against normality for the term spread in this extended second sub-sample. Table A3 reports the in sample RMSE and KLIC based measures of fit. The results are very similar to those reported in Tables 3 and 4 of the main text.

\subsection{Common Dependence Across Eras}

In this appendix we report results based on era specific marginal distributions, either gaussian or non-gaussian, but common dependence, either linear or non-linear across all eras. Our use of era specific margins is motivated by the differences in the means and standard deviations of the data across eras. Table A4 reports the in sample RMSE and KLIC based measures of fit. As with the results reported in Tables 3 and 4 of the main text we see gains in both measures of fit with the inclusion of financial conditions in the specifications that allow for non-linear dependence. However, these gains from adding financial conditions are not as great as those reported in the main text where we allow dependence to vary across eras.

\subsection{Plots for Alternative Financial Variables}

Figures A4a to A4i plot the means of the conditional distributions and the 5th and 95th percentiles. For the sub-sample 1875 to 1913 financial conditions are measured by the credit spread (figure A4a) and stock market volatility (figure A4b). For the sub-sample 1919 to 1941 financial conditions are measured by the term spread (figure A4c) and stock market volatility (figure A4d), and by the same two variables for the sub-sample 1946 to 1971 (figures A4e and A4f respectively). Finally for the modern era, 1971 - 2017, financial conditions are measured by the term spread (figure A4g), the credit spread (figure A4h) and stock market volatility (figure A4i). The results in this appendix are consistent with those in the paper, that is, with the addition of a measure of financial conditions, the conditional mean of the distribution for output growth tracks the out-turn more closely.

Figures A5a to A5i plot the conditional probabilities of the low and high growth events for alternative measures of financial conditions. We use the same definition of the high and low growth events that we use in the main text, namely a growth rates in the top and bottom decile respectively. As we find in the main text, the addition of financial conditions greatly sharpens the signal about the likelihood of both events.

Figure A6 shows predictive densities for the quarter and for the year following our four selected financial crises using alternative measures of financial conditions. For the 1893 crisis we see a similar conditional density for the credit spread and stock market volatility, with much more probability mass in the negative range of the distribution. For the two measures used in this appendix we see slightly less evidence of bi-modality at the one quarter horizon that we see for the term spread, but for the one year horizon there is much more evidence of bi-modality when we represent financial conditions by the credit spread or stock market volatility. For the 1907 crisis the addition of financial conditions leads to more pessimistic predictive density for the quarter after the crisis, but does not have much of an impact on 
the probability of negative growth in the year after the crisis. This is the case when using the term spread, and when using either the credit spread or stock market volatility. We see some evidence of bi-modality at the one year horizon in this appendix when we use stock market volatility, which is absent when we use the term spread. The addition of financial conditions leads to a considerable increase in the probability of negative growth both in the quarter and the year after the 1929 stock market crash. Finally, for the 2008 crisis, we generally see a slight movement to the left of the predictive density with the addition of financial variables. This is similar to what we see with the NFCI in the paper.

\subsection{Probability Plots for Alternative Definitions of Low and High Growth}

In this appendix we consider alternative definitions of low and high growth events. We consider a low growth event as occurring when output growth is negative and a high growth event as occurring when output growth is above twice the corresponding sub-sample mean. Probabilities for these events are reported in figures $8 \mathrm{a}$ to $8 \mathrm{~d}$. These plots indicate a much sharper signal about the probabilities of the events when we also condition on financial conditions.

\section{References}

Nathan S. Balke and Robert J. Gordon (1986) "Appendix B: Historical Data" in Robert J. Gordon ed. The American Business Cycle: Continuity and Change University of Chicago Press, Chicago.

Macaulay, Frederick R. (1938) The Movement of Interest Rates, Bond Yields and Stock Prices in the United States Since 1856 NBER.

Shiller, Robert J. (2000) Irrational Exhuberence, Princeton University Press, Princeton. 
Table A1: Out of Sample Forecast Performance and Bandwidth

\begin{tabular}{lcccc}
\hline & \multicolumn{2}{c}{ Root Mean Squared Errors } & \multicolumn{2}{c}{ KLIC Based Measure } \\
Bandwidth & 1 Quarter Growth & 4 Quarter Growth & 1 Quarter Growth & 4 Quarter Growth \\
\hline & & & & \\
$h=0.0001$ & 1.759 & 1.790 & 5.251 & 5.270 \\
$h=0.001$ & 1.017 & 1.063 & 1.041 & 1.230 \\
$h=0.005$ & 1.007 & 1.029 & 1.007 & 1.027 \\
$h=0.01$ & 1.000 & 1.000 & 1.000 & 1.000 \\
$h=0.02$ & 0.993 & 0.967 & 0.995 & 0.983 \\
$h=0.04$ & 0.988 & 0.937 & 0.989 & 0.970 \\
$h=0.08$ & 0.990 & 0.929 & 0.985 & 0.961 \\
$h=0.16$ & 0.997 & 0.956 & 0.984 & 0.960 \\
$h=0.32$ & 1.006 & 1.004 & 0.986 & 0.964 \\
$h=0.64$ & 1.014 & 1.047 & 0.989 & 0.970 \\
$h=1.28$ & 1.022 & 1.071 & 0.991 & 0.974 \\
\hline
\end{tabular}

Note: Root mean squared errors and KLIC based measure of fit are reported relative to the copula using a bandwidth of $h=0.01$.

Table A2: Summary Statistics - Alternative Second Sub-Sample 1914:1 to 1945:4

\begin{tabular}{lcccccc}
\hline & Mean & $\begin{array}{c}\text { Standard } \\
\text { Deviation }\end{array}$ & Skewness & Kurtosis & $\begin{array}{c}\text { Shapiro-Wilk } \\
p \text {-value }\end{array}$ & $\begin{array}{c}\text { Number of } \\
\text { Observations }\end{array}$ \\
\cline { 2 - 6 } & & & & & & \\
1 Quarter Growth Rate & 3.15 & 12.6 & -0.51 & 2.84 & 0.011 & 127 \\
4 Quarter Growth Rate & 3.85 & 9.11 & -0.38 & 2.25 & 0.002 & 124 \\
Term Spread & 2.74 & 1.93 & 0.14 & 2.63 & 0.728 & 128 \\
Credit Spread & 1.87 & 0.80 & 1.60 & 7.70 & $<0.001$ & 128 \\
Stock Market Volatility & 3.74 & 3.18 & 3.13 & 18.2 & $<0.001$ & 128 \\
& & & & & & \\
\hline
\end{tabular}

Notes: 1 Quarter growth rates are reported at an annualized rate. The Shapiro-Wilk $p$-value refers to a test of the null hypothesis that the the variable is Gaussian distributed. 


\section{Table A3: Root Mean Square Error and KLIC-Based Measures of Predictive Accuracy For Alternative Sub-Sample}

\begin{tabular}{|c|c|c|c|c|}
\hline & \multicolumn{2}{|c|}{ Root Mean Squared Errors } & \multicolumn{2}{|c|}{ KLIC Based Measure } \\
\hline & 1 Qtr Growth & 4 Qtr Growth & 1 Qtr Growth & 4 Qtr Growth \\
\hline & 1914:2-1945:4 & 1915:1-1945:4 & 1914:2-1945:4 & 1915:1-1945:4 \\
\hline \multicolumn{5}{|c|}{ Gaussian Marginals and Linear Dependence } \\
\hline No Financial Conditions & 1.000 & 1.000 & 1.000 & 1.000 \\
\hline Term Spread & 0.994 & $1.038^{*}$ & 0.998 & 1.001 \\
\hline Credit Spread & 0.999 & 1.029 & 0.999 & 1.001 \\
\hline S\&P Volatility & 1.000 & 1.000 & 1.000 & 0.999 \\
\hline \multicolumn{5}{|c|}{ Non-Gaussian Marginals and Linear Dependence } \\
\hline No Financial Conditions & 1.001 & $0.883^{* * *}$ & $0.993^{*}$ & $0.965^{* *}$ \\
\hline Term Spread & 0.995 & $0.866^{* * *}$ & $0.991^{*}$ & $0.960^{* * *}$ \\
\hline Credit Spread & 0.999 & $0.873^{* * *}$ & $0.992^{*}$ & $0.963^{* *}$ \\
\hline S\&P Volatility & 1.001 & $0.881^{* * *}$ & $0.992^{*}$ & $0.964^{* *}$ \\
\hline \multicolumn{5}{|c|}{ Gaussian Marginals and Non-linear Dependence } \\
\hline No Financial Conditions & $0.951^{*}$ & $0.857^{* * *}$ & $0.953^{* * *}$ & $0.939^{* * *}$ \\
\hline Term Spread & $0.734^{* * *}$ & $0.635^{* * *}$ & $0.836^{* * *}$ & $0.822^{* * *}$ \\
\hline Credit Spread & $0.753^{* * *}$ & $0.666^{* * *}$ & $0.847^{* * *}$ & $0.845^{* * *}$ \\
\hline S\&P Volatility & $0.713^{* * *}$ & $0.729^{* * *}$ & $0.863^{* * *}$ & $0.879^{* * *}$ \\
\hline \multicolumn{5}{|c|}{ Non-Gaussian Marginals and Non-linear Dependence } \\
\hline No Financial Conditions & $0.940^{* *}$ & $0.862^{* * *}$ & $0.955^{* * *}$ & $0.938^{* * *}$ \\
\hline Term Spread & $0.718^{* * *}$ & $0.586^{* * *}$ & $0.831^{* * *}$ & $0.803^{* * *}$ \\
\hline Credit Spread & $0.679^{* * *}$ & $0.585^{* * *}$ & $0.830^{* * *}$ & $0.806^{* * *}$ \\
\hline S\&P Volatility & $0.598^{* * *}$ & $0.585^{* * *}$ & $0.819^{* * *}$ & $0.819^{* * *}$ \\
\hline
\end{tabular}

Notes: (1) Root Mean Squared Errors are reported relative to the benchmark model with Gaussian marginals, linear dependence and no financial conditions. As a rough guide to statistical significance, pvalues of a Harvey, Leybourne, and Newbold (1997) small-sample adjustment of the two-sided Diebold and Mariano (1995) test are denoted by $*(10 \%), * *(5 \%)$ and $* * *(1 \%)$. (2) The KLIC based measures are average log scores relative to the benchmark model with Gaussian marginals, linear dependence and no financial conditions. As a rough guide to statistical significance, p-values of a two-sided DieboldMariano (1995) type test for the log score are denoted by $*(10 \%), * *(5 \%)$ and $* * *(1 \%)$. 


\section{Table A4: Root Mean Square Error and KLIC-Based Measures of Predictive Accuracy For Specification with Common Dependence Across Sub-Samples}

\begin{tabular}{|c|c|c|c|c|}
\hline & \multicolumn{2}{|c|}{ Root Mean Squared Errors } & \multicolumn{2}{|c|}{ KLIC Based Measure } \\
\hline & 1-Qtr Growth & 4-Qtr Growth & 1-Qtr Growth & 4-Qtr Growth \\
\hline \multicolumn{5}{|c|}{ Gaussian Marginals and Linear Dependence } \\
\hline No Financial Conditions & 1.000 & 1.000 & 1.000 & 1.000 \\
\hline Term Spread & 0.984 & $0.955^{* *}$ & $0.992^{* *}$ & $0.983^{* * *}$ \\
\hline Credit Spread & 1.002 & 0.997 & 0.997 & 1.000 \\
\hline S\&P Volatility & 0.995 & 0.998 & $0.996^{*}$ & 0.998 \\
\hline \multicolumn{5}{|c|}{ Non-Gaussian Marginals and Linear Dependence } \\
\hline No Financial Conditions & 1.002 & 1.001 & $0.983^{* * *}$ & $0.989^{* * *}$ \\
\hline Term Spread & 0.986 & $0.953^{*}$ & $0.978^{* * *}$ & $0.974^{* * *}$ \\
\hline Credit Spread & 1.001 & 0.999 & $0.982^{* * *}$ & $0.990^{* * *}$ \\
\hline S\&P Volatility & 0.997 & 1.000 & $0.981^{* * *}$ & $0.989^{* * *}$ \\
\hline \multicolumn{5}{|c|}{ Gaussian Marginals and Non-linear Dependence } \\
\hline No Financial Conditions & 0.989 & $0.984^{*}$ & 0.984 & $0.982^{* * *}$ \\
\hline Term Spread & $0.905^{* * *}$ & $0.844^{* * *}$ & $0.894^{* * *}$ & $0.878^{* * *}$ \\
\hline Credit Spread & $0.926^{* * *}$ & $0.897^{* * *}$ & $0.909^{* * *}$ & $0.898^{* * *}$ \\
\hline S\&P Volatility & $0.937^{* * *}$ & $0.926^{* * *}$ & $0.911^{* * *}$ & $0.917^{* * *}$ \\
\hline \multicolumn{5}{|c|}{ Non-Gaussian Marginals and Non-linear Dependence } \\
\hline No Financial Conditions & 0.997 & 0.986 & $0.968^{* * *}$ & $0.968^{* * *}$ \\
\hline Term Spread & $0.916^{* * *}$ & $0.854^{* * *}$ & $0.881^{* * *}$ & $0.862^{* * *}$ \\
\hline Credit Spread & $0.948^{* * *}$ & $0.909^{* * *}$ & $0.881^{* * *}$ & $0.869^{* * *}$ \\
\hline S\&P Volatility & $0.917^{* * *}$ & $0.912^{* * *}$ & $0.879^{* * *}$ & $0.876^{* * *}$ \\
\hline
\end{tabular}

Notes: (1) Our sample for 1-quarter growth is 1875Q3-1913Q4, 1919Q2-1941Q3, 1946Q2-1971Q2 and 1971Q4 to 2017Q4, and our sample for 4-quarter growth is 1877Q1-1913Q4, 1920Q1-1941Q3, 1947Q11971Q2 and 1972Q3 to 2017Q4. (2) Root Mean Squared Errors are reported relative to the benchmark model with Gaussian marginals, linear dependence and no financial conditions. As a rough guide to statistical significance, p-values of a Harvey, Leybourne, and Newbold (1997) small-sample adjustment of the two-sided Diebold and Mariano (1995) test are denoted by $*(10 \%), * *(5 \%)$ and $* * *(1 \%)$. (3) The KLIC based measures are average log scores relative to the benchmark model with Gaussian marginals, linear dependence and no financial conditions. As a rough guide to statistical significance, p-values of a two-sided Diebold-Mariano (1995) type test for the log score are denoted by $*(10 \%), * *$ $(5 \%)$ and $* * *(1 \%)$. 
Figure A1a: Annualized 1-Quarter Output Growth
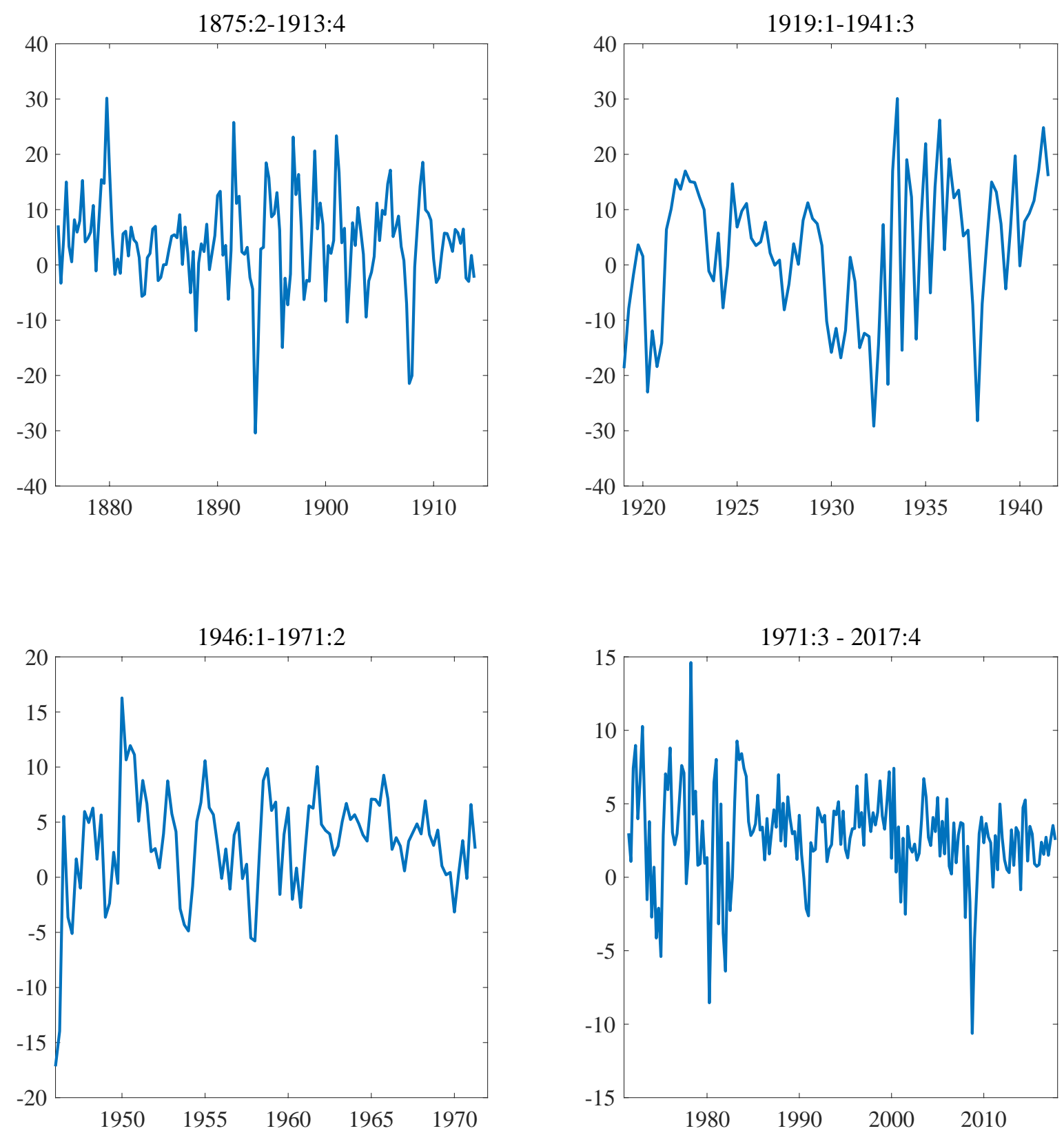
Figure A1b: 4-Quarter Output Growth
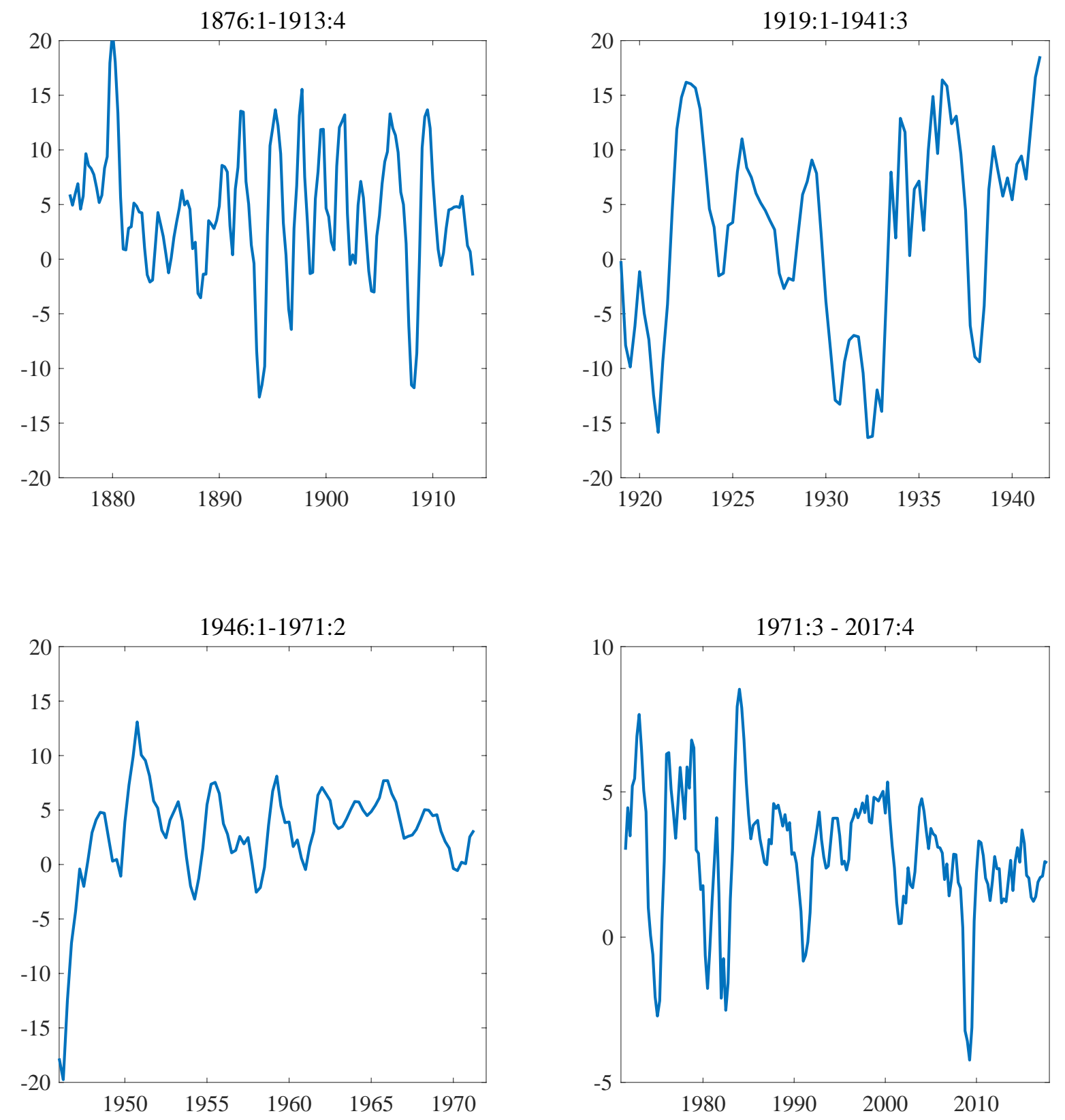
Figure A1c: Term Spread
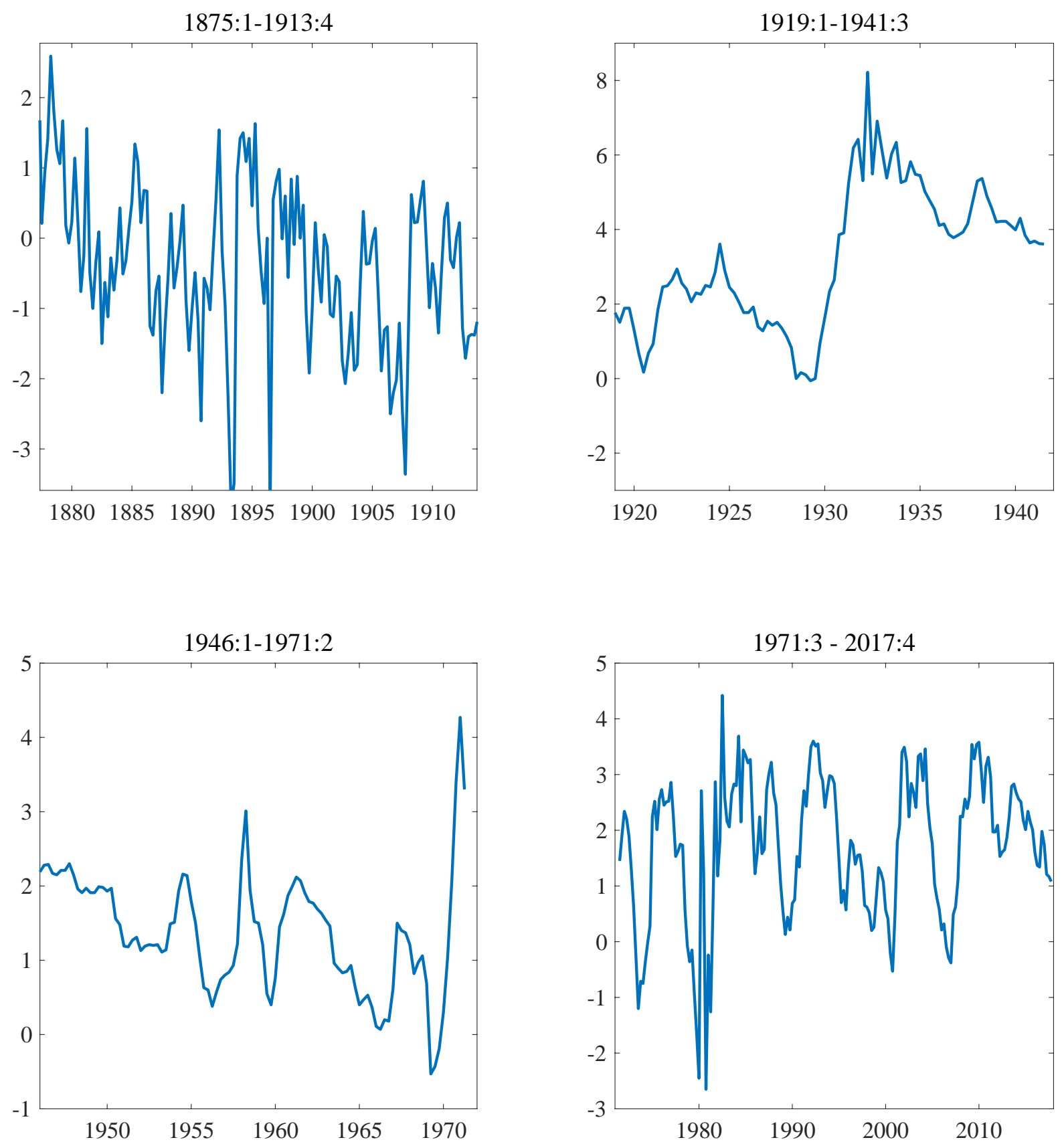
Figure A1d: Credit Spread
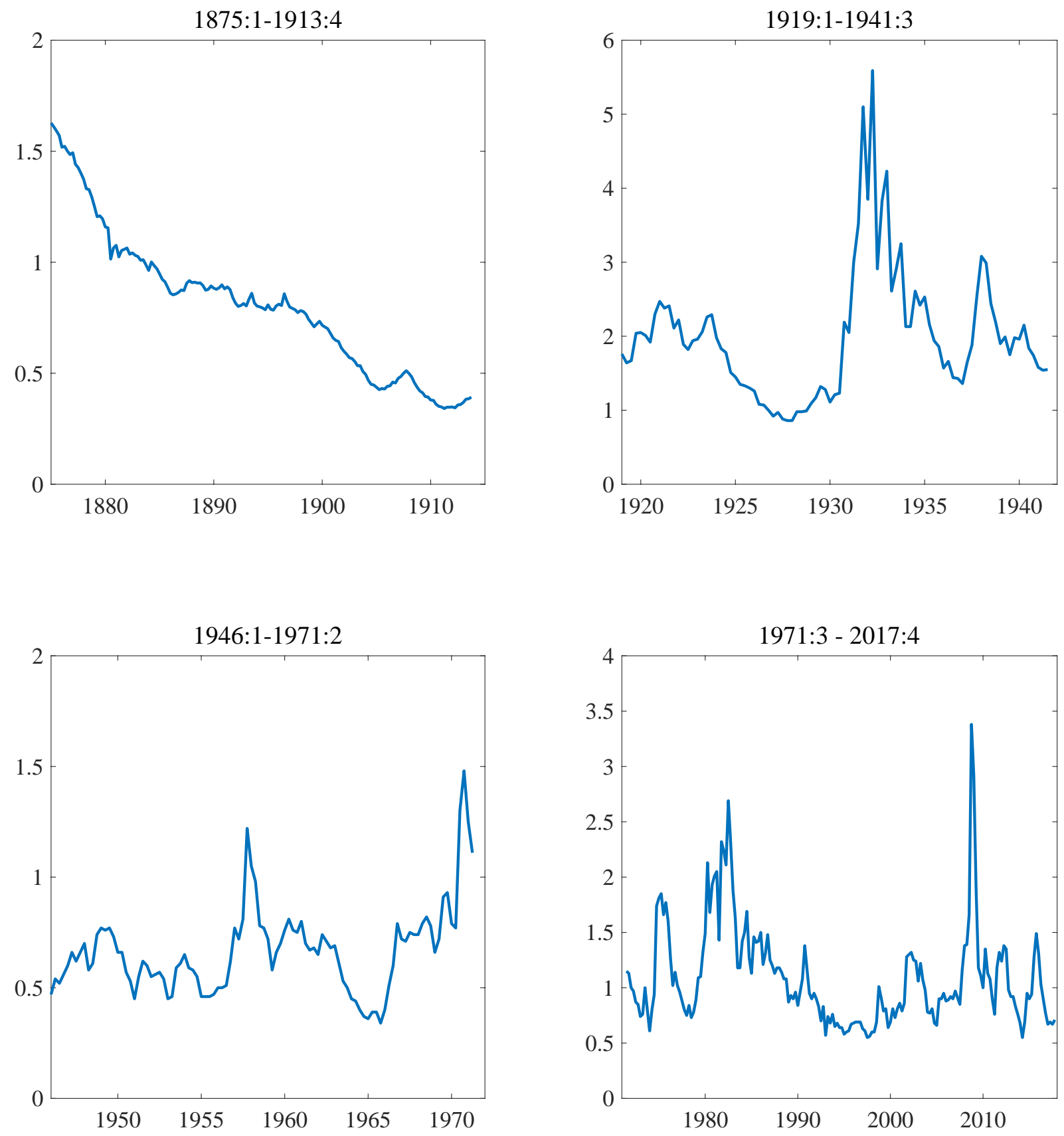
Figure A1e: S\&P Volatility
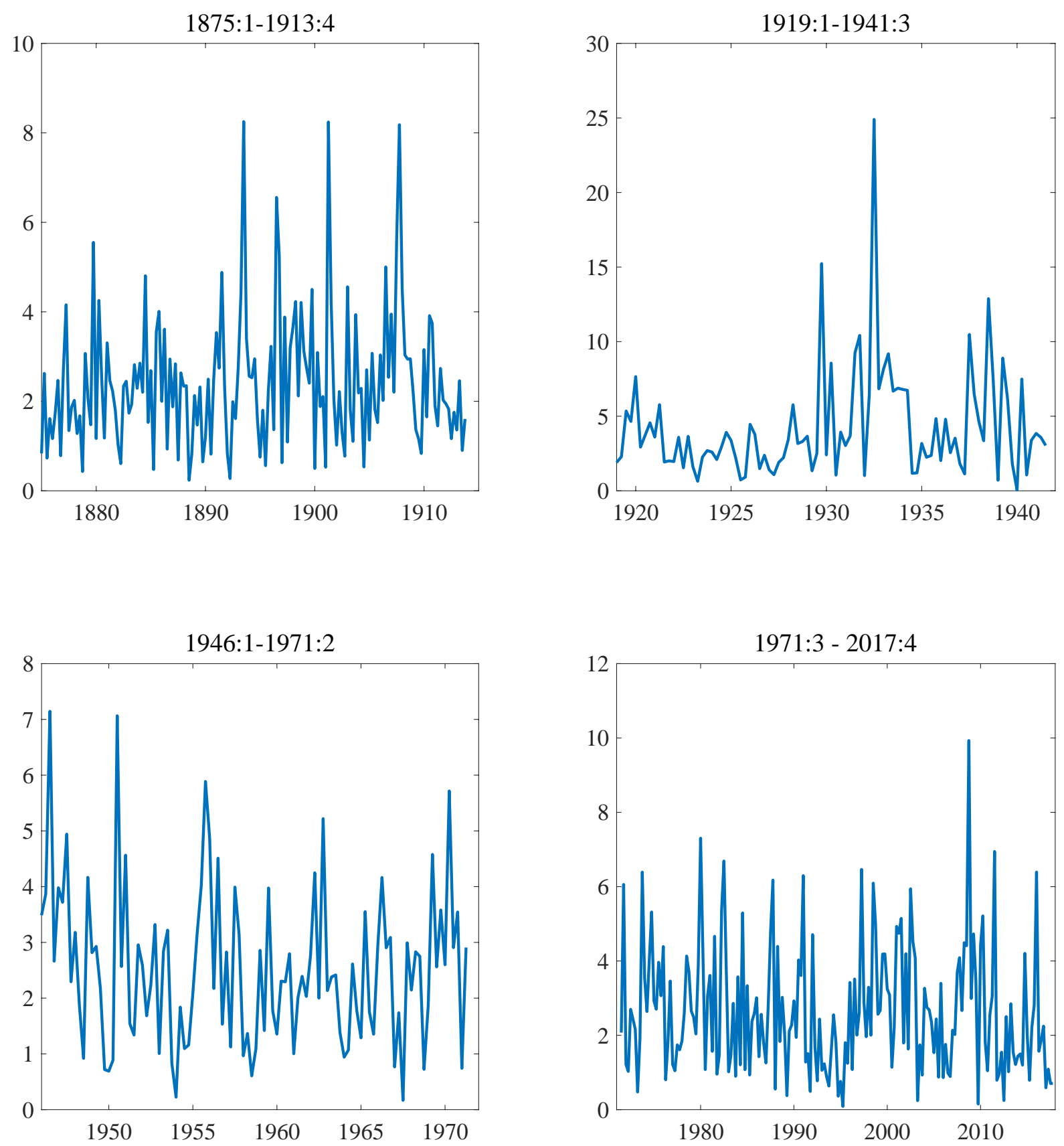
Figure A1f: National Financial Conditions Index

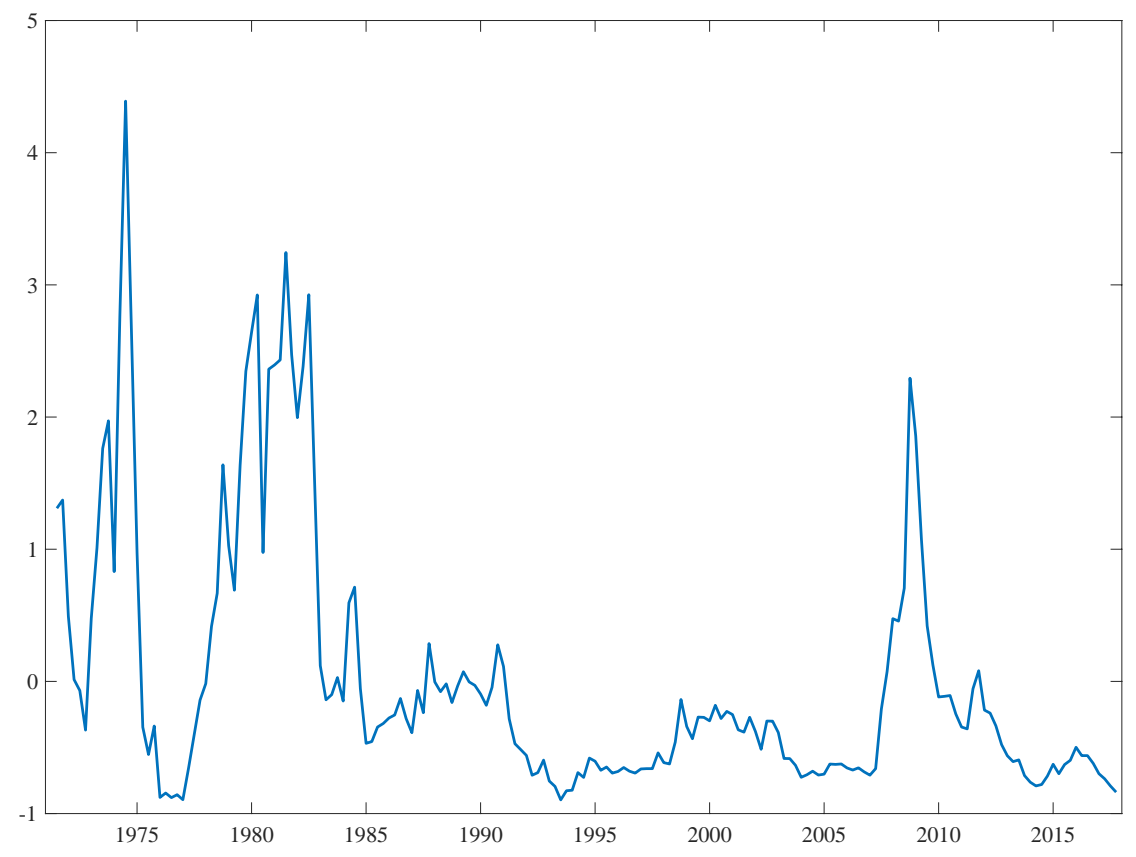

Figure A2: Alternative Credit Spreads for Overlap

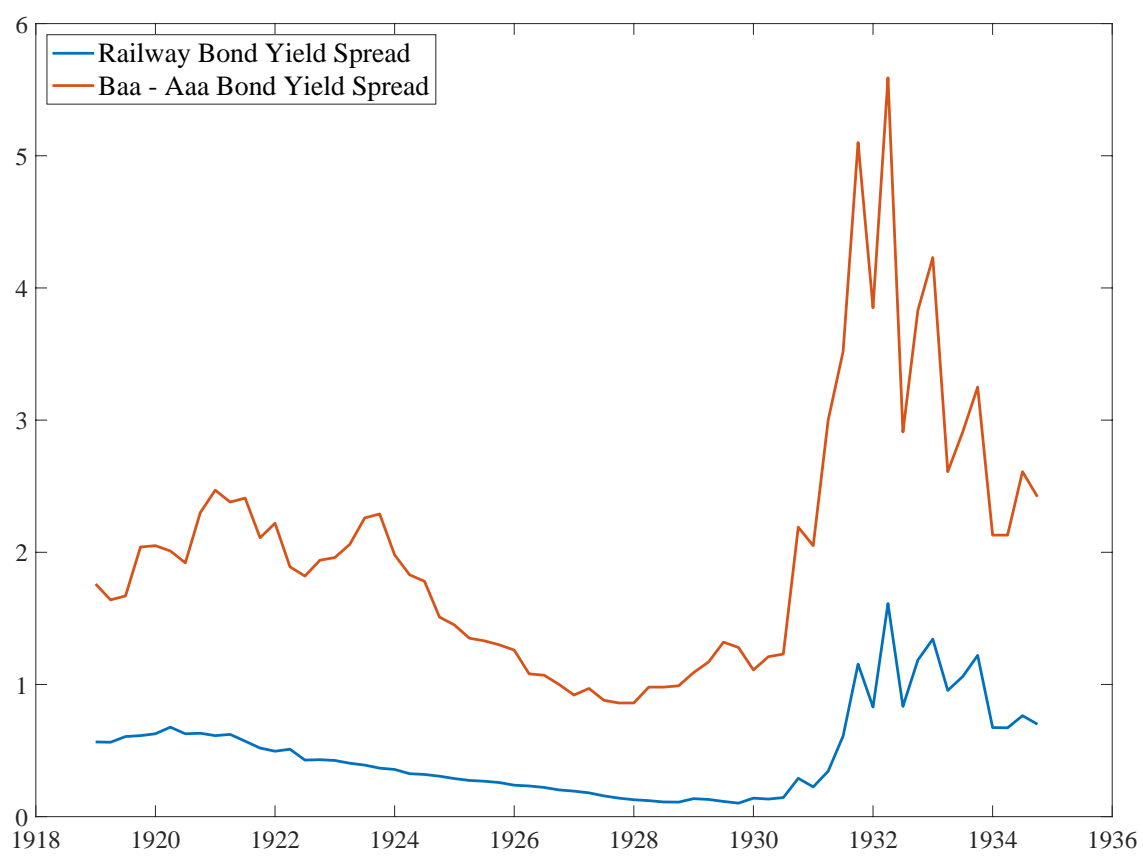


Figure A3a: Recursive Measures of Fit 1875 - 1913
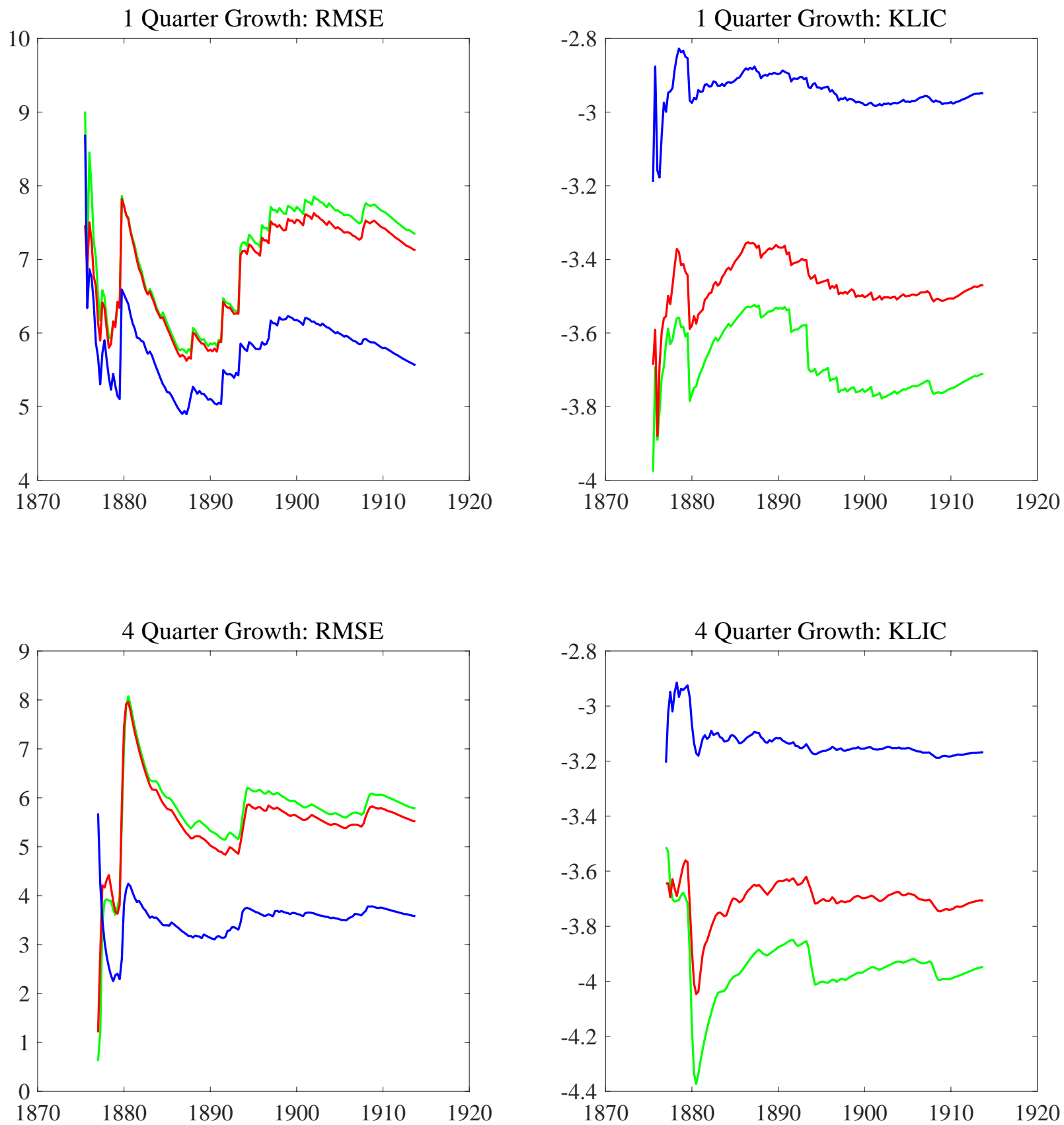

Note: The green line represents the measure of fit from the specification with Gaussian marginals and linear dependence, but no measure of financial conditions. The red and blue lines plot our preferred specification with (blue) and without (red) financial conditions, the term spread. 
Figure A3b: Recursive Measures of Fit 1919 - 1941
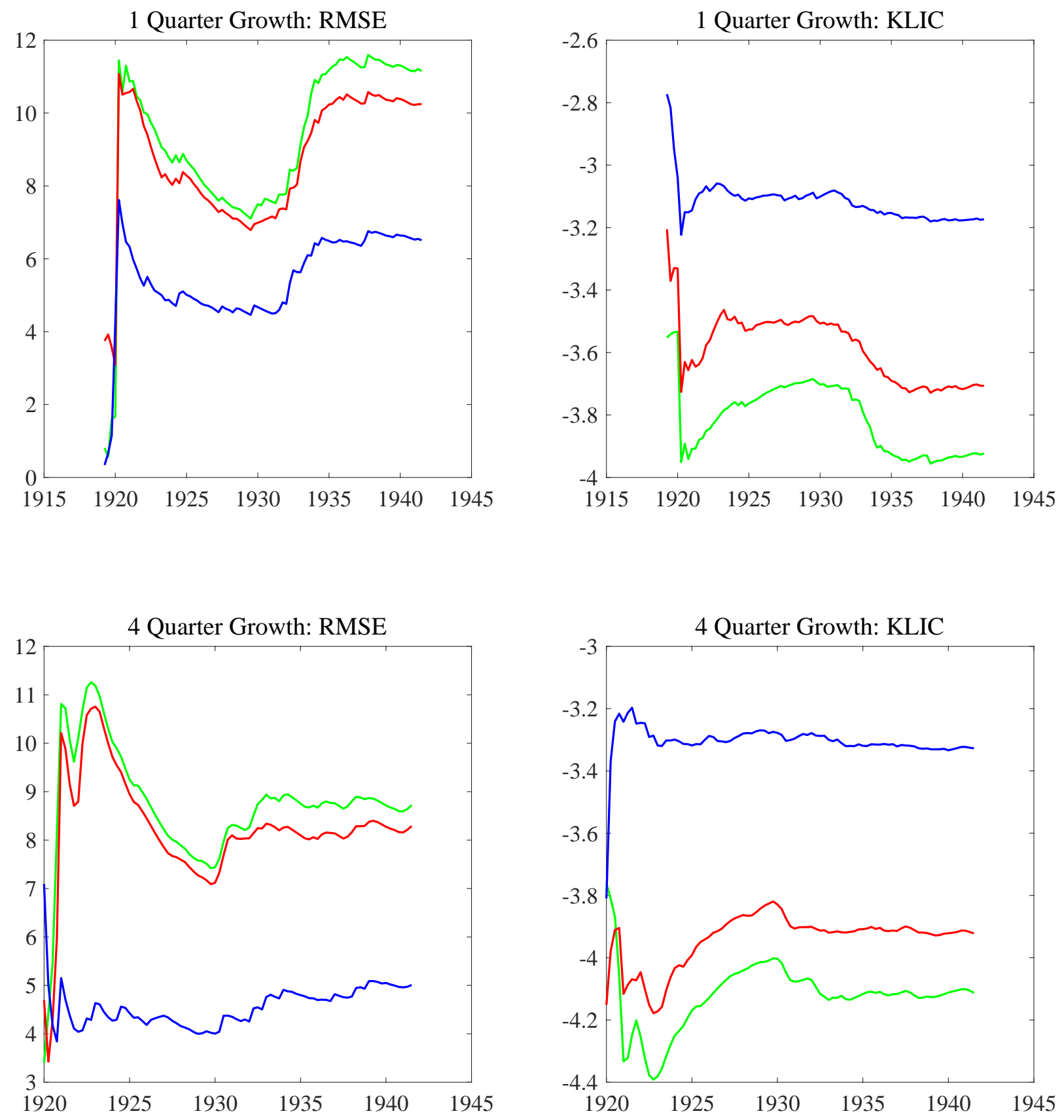

Note: The green line represents the measure of fit from the specification with Gaussian marginals and linear dependence, but no measure of financial conditions. The red and blue lines plot our preferred specification with (blue) and without (red) financial conditions, the credit spread. 
Figure A3c: Recursive Measures of Fit 1946 - 1971
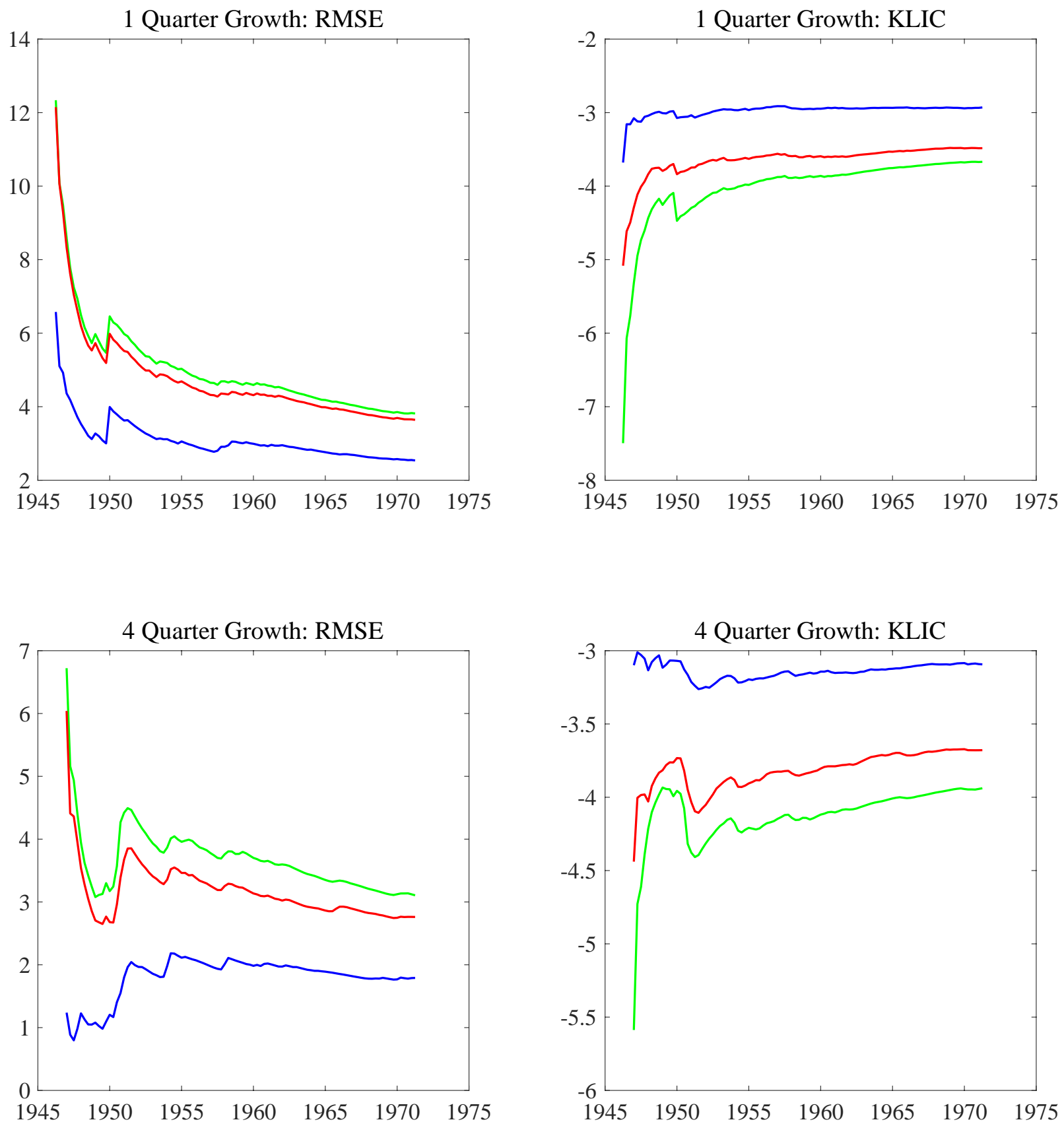

Note: The green line represents the measure of fit from the specification with Gaussian marginals and linear dependence, but no measure of financial conditions. The red and blue lines plot our preferred specification with (blue) and without (red) financial conditions, the credit spread. 
Figure A3d: Recursive Measures of Fit 1971 - 2017
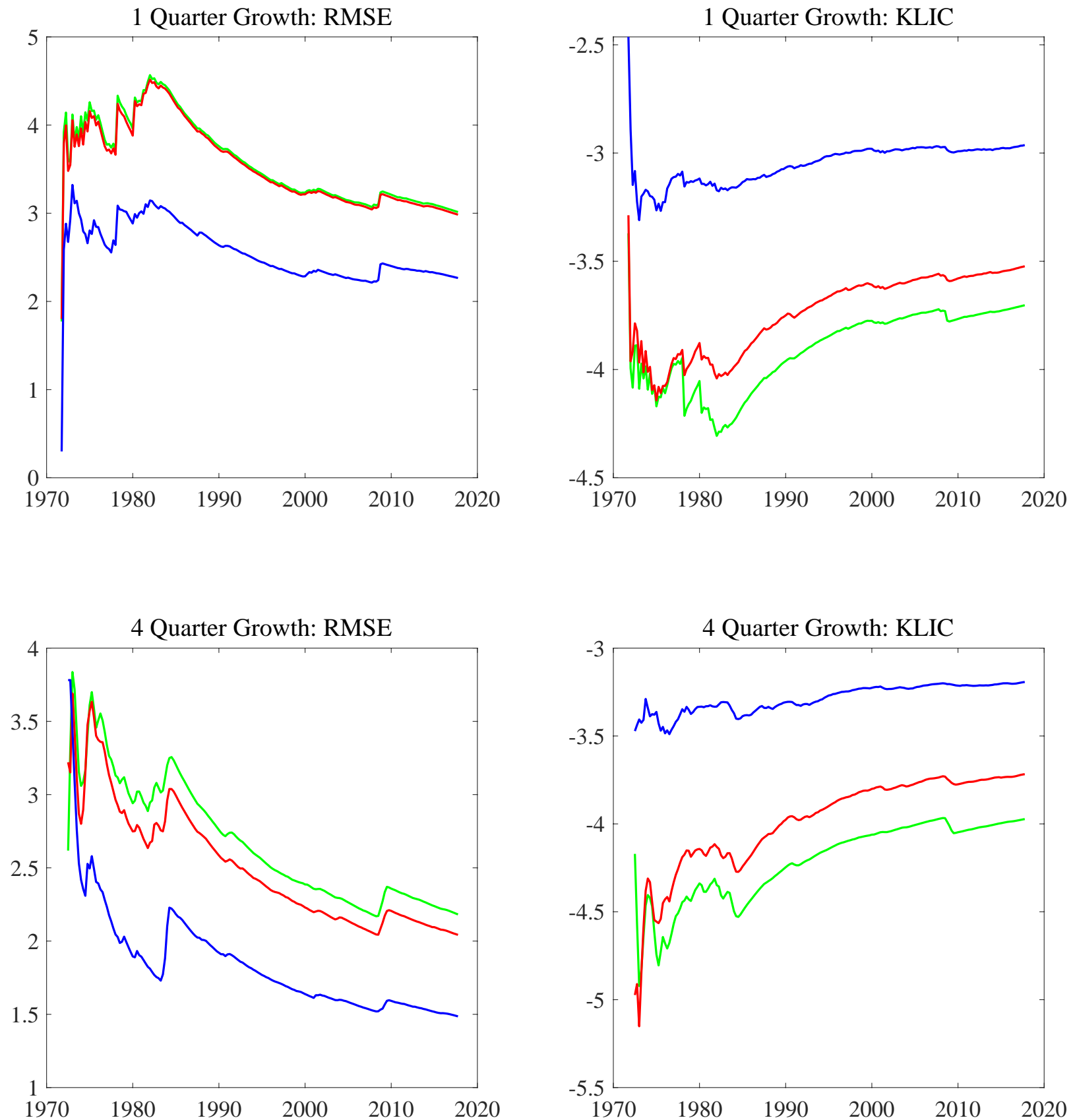

Note: The green line represents the measure of fit from the specification with Gaussian marginals and linear dependence, but no measure of financial conditions. The red and blue lines plot our preferred specification with (blue) and without (red) financial conditions, the NFCI. 
Figure A4a: Conditional Densities 1875 - 1913
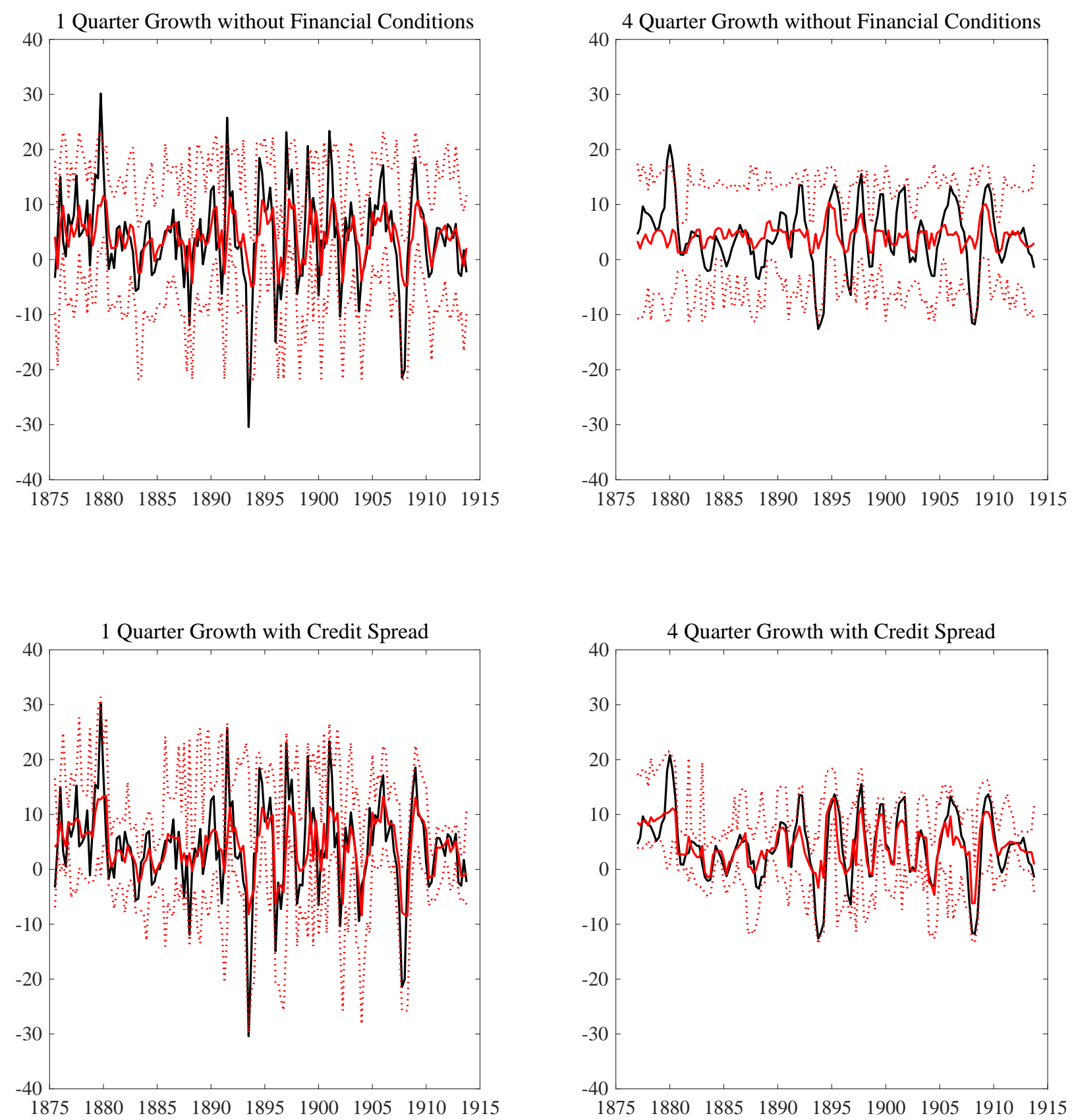

Note: The solid line black line depicts the realizations and the solid red line depicts the mean of the conditional density for output growth based on a model with non-Gaussian marginals and non-linear dependence. The dotted red lines depict the $5^{\text {th }}$ and $95^{\text {th }}$ percentiles. 
Figure A4b: Conditional Densities 1875 - 1913
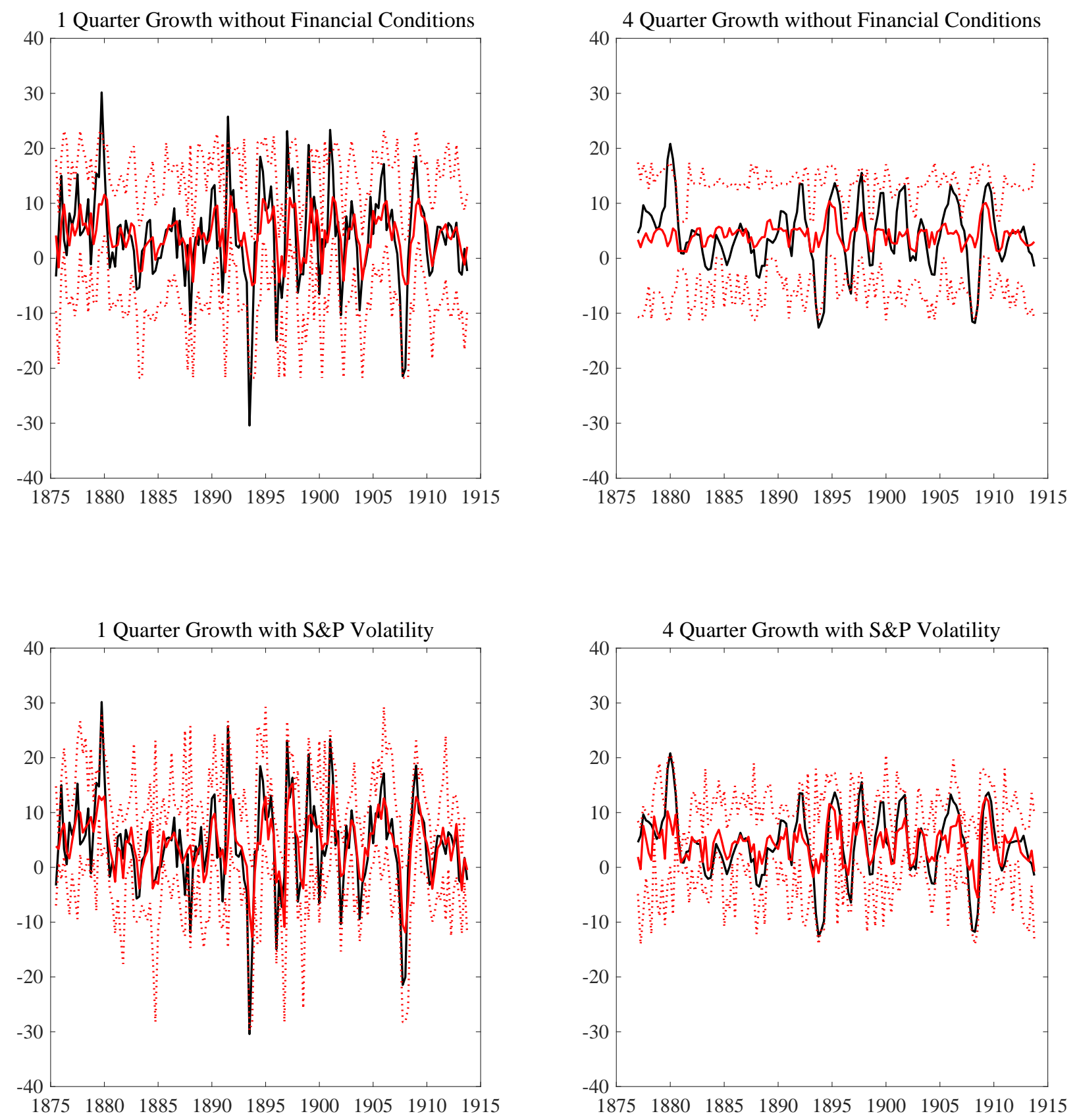

Note: See note to Figure A4a. 
Figure A4c: Conditional Densities 1919 - 1941
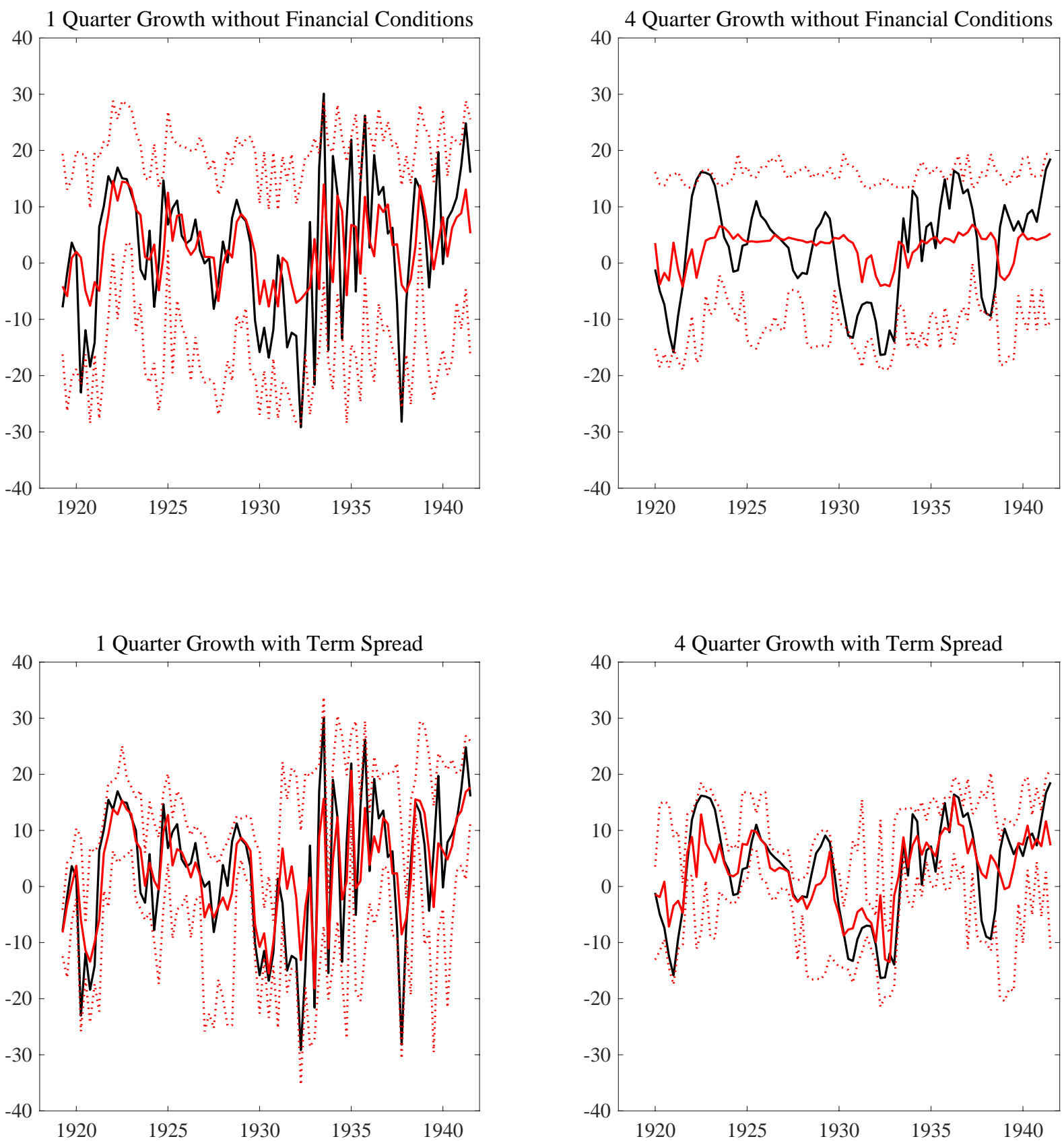

Note: See note to Figure A4a. 
Figure A4d: Conditional Densities 1919 - 1941
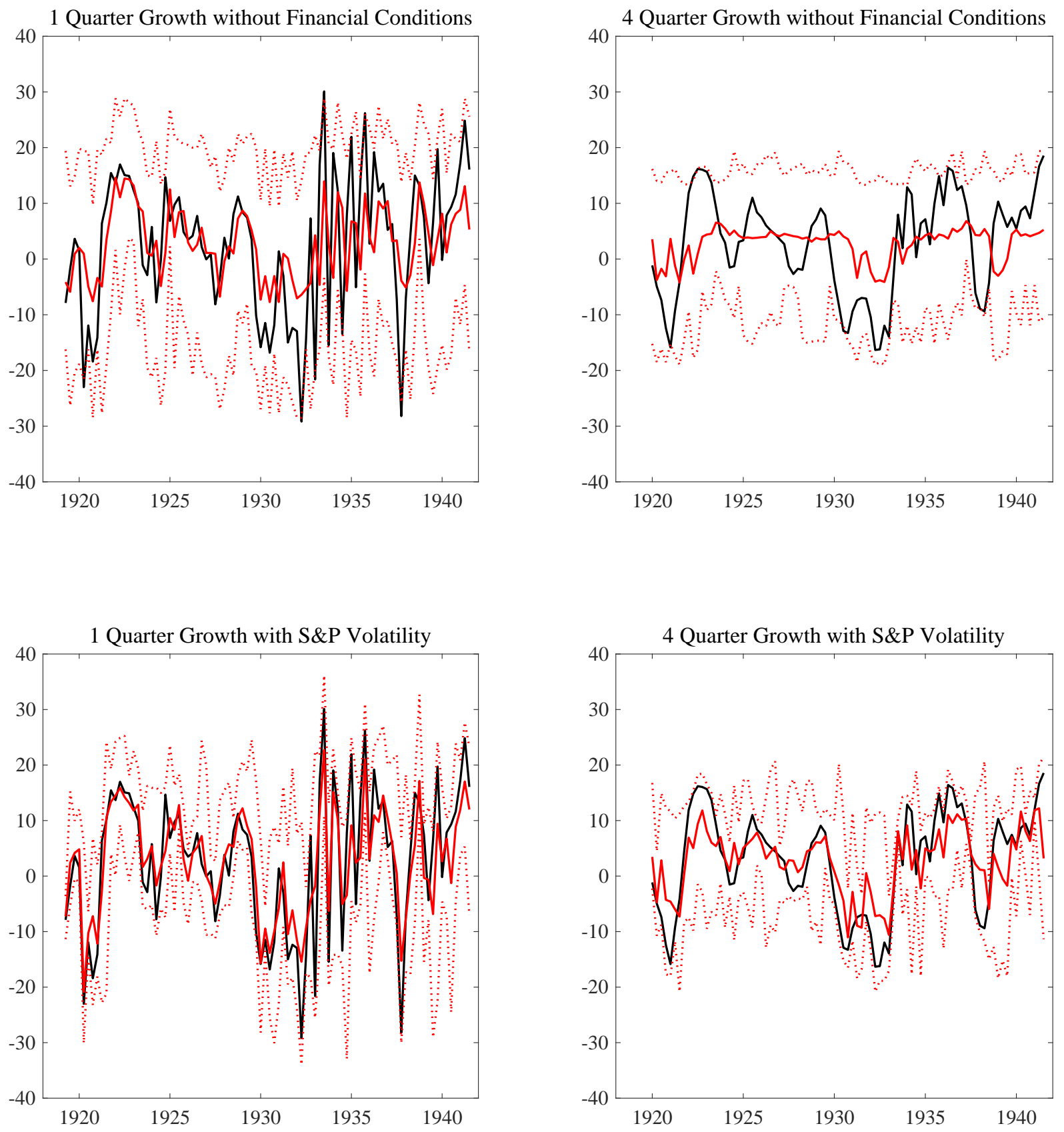

Note: See note to Figure A4a. 
Figure A4e: Conditional Densities 1946 - 1971
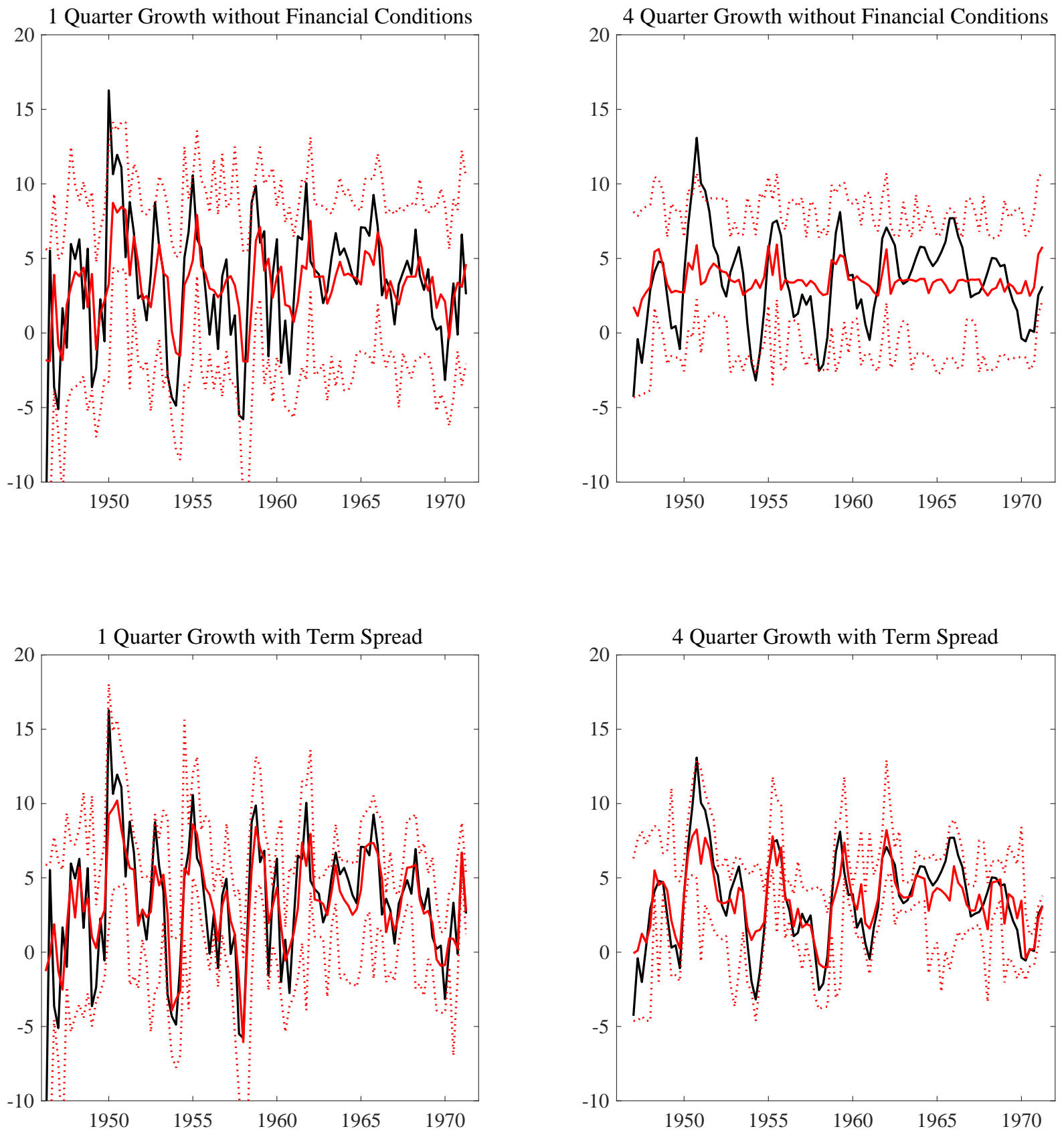

Note: See note to Figure A4a. 
Figure A4f: Conditional Densities 1946 - 1971
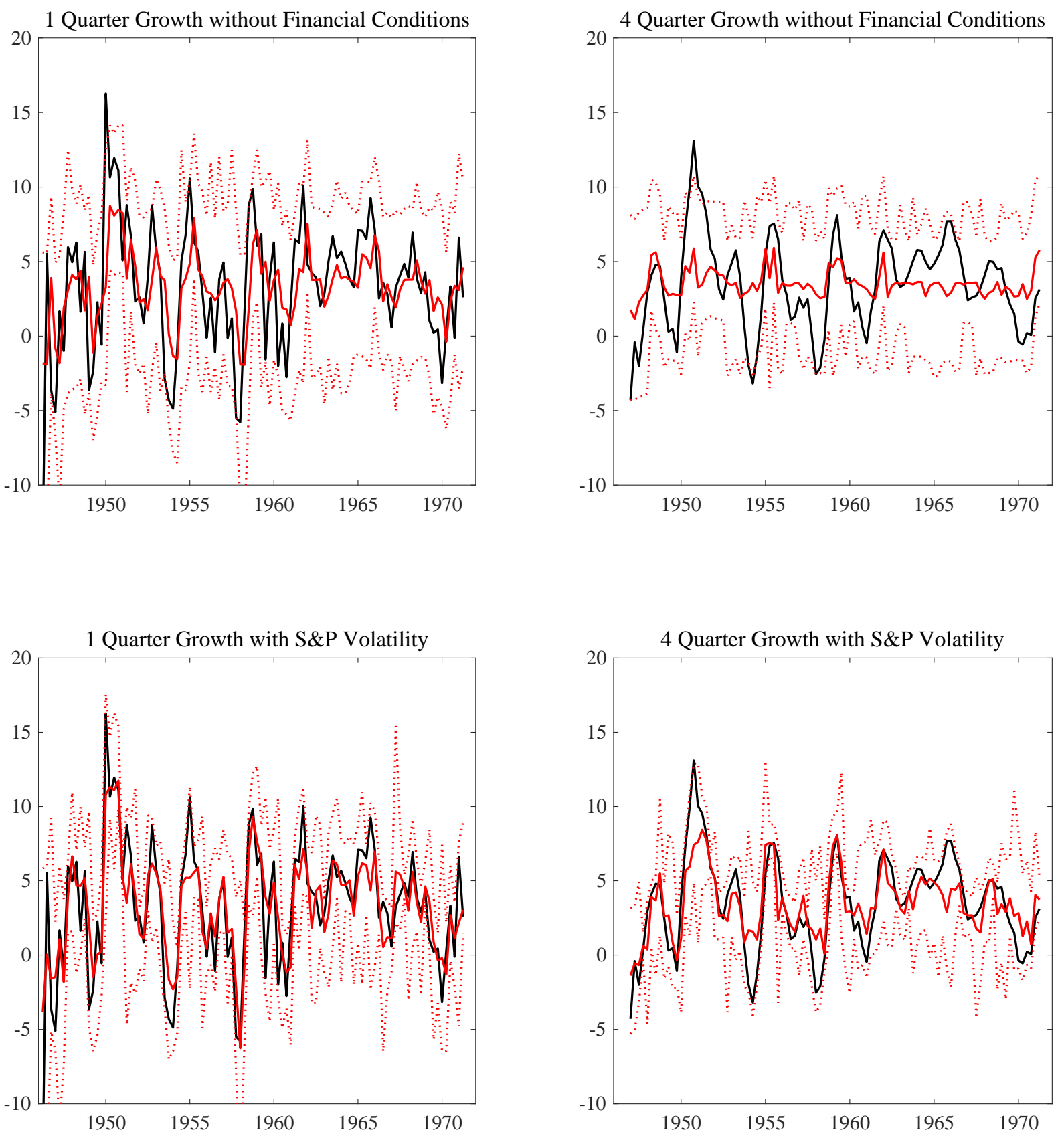

Note: See note to Figure A4a. 
Figure A4g: Conditional Densities 1971 - 2017
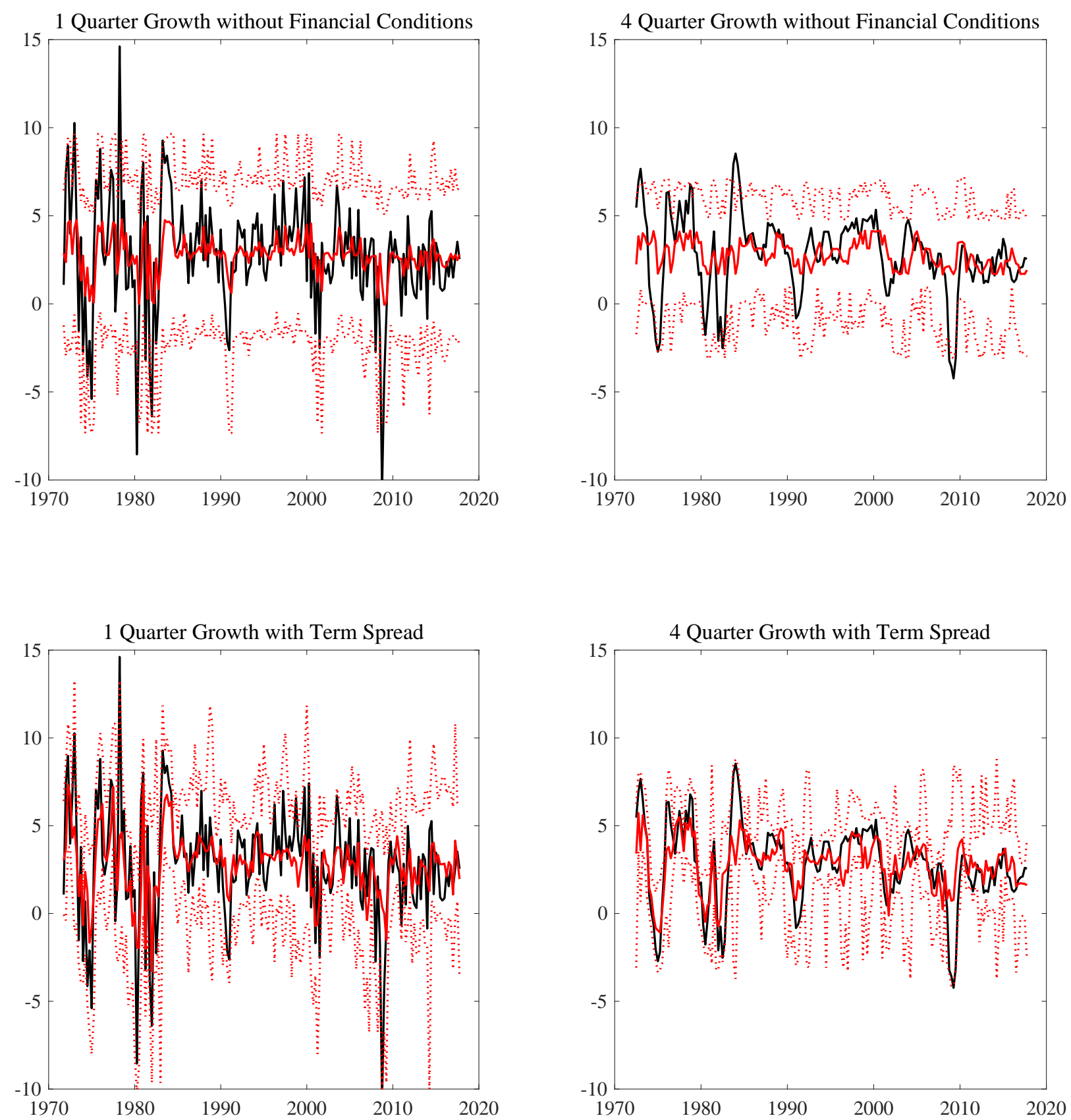

Note: See note to Figure A4a. 
Figure A4h: Conditional Densities 1971 - 2017
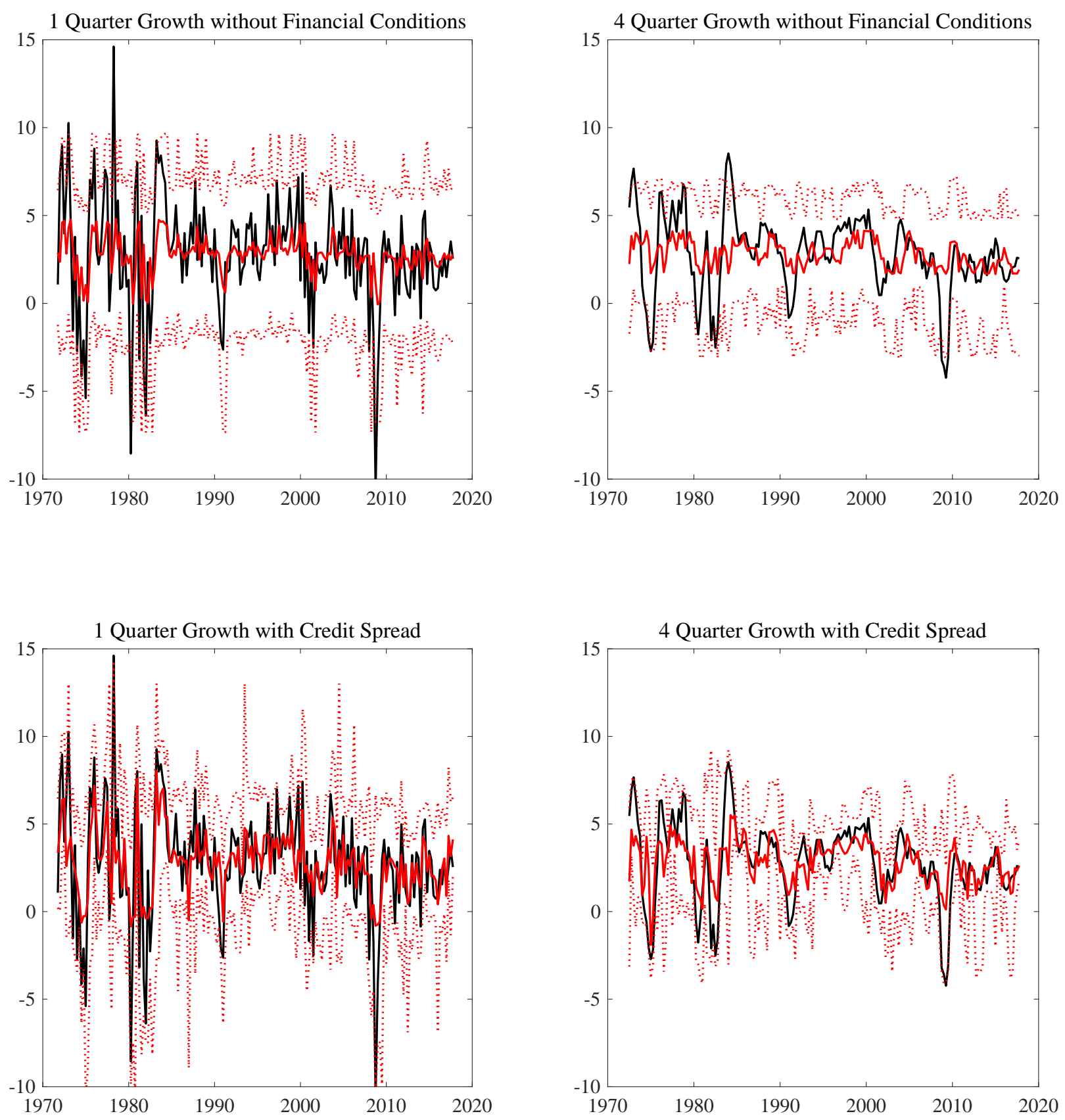

Note: See note to Figure A4a. 
Figure A4i: Conditional Densities 1971 - 2017
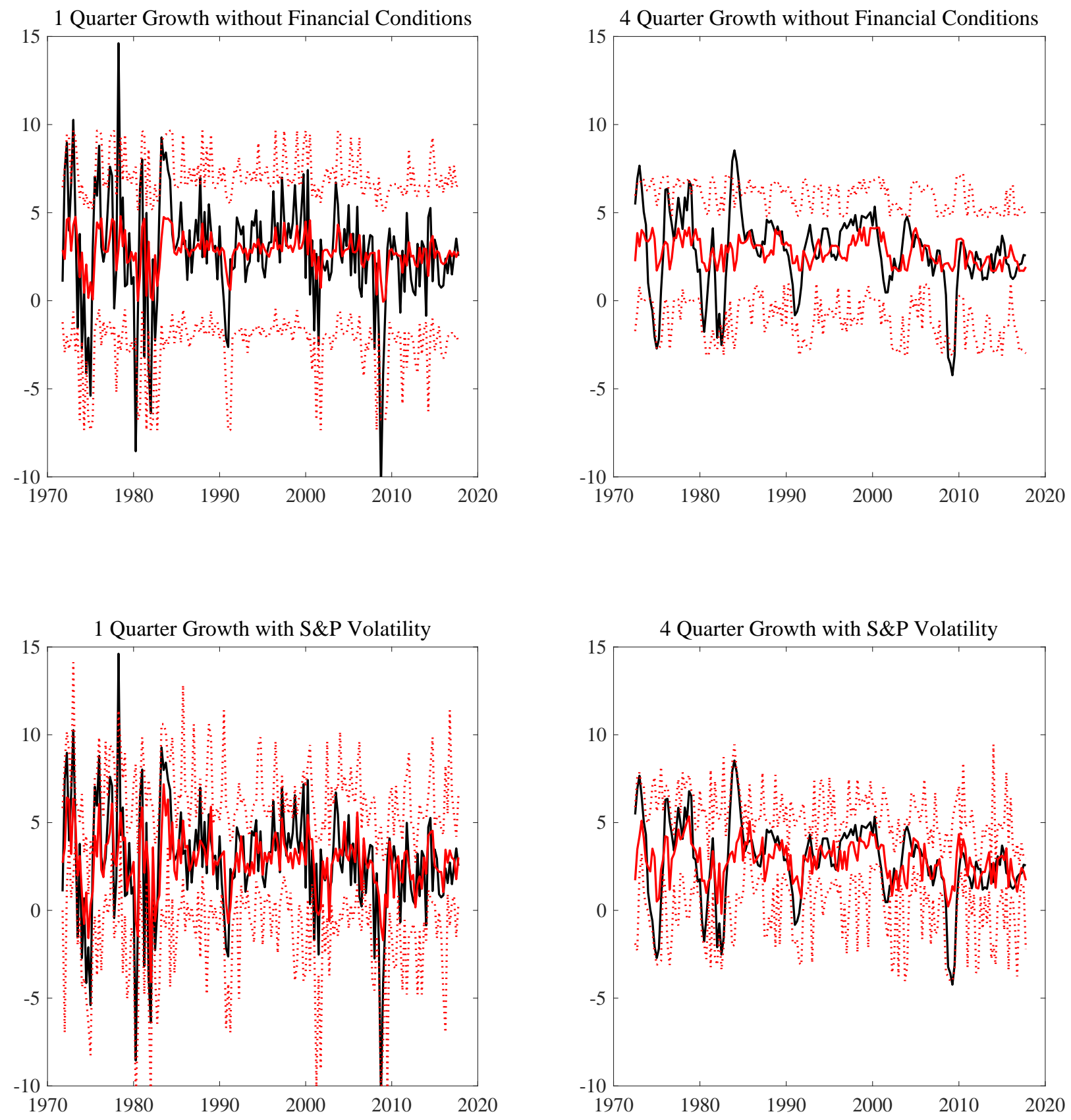

Note: See note to Figure A4a. 
Figure A5a: Probability of Tail Events 1875 - 1913
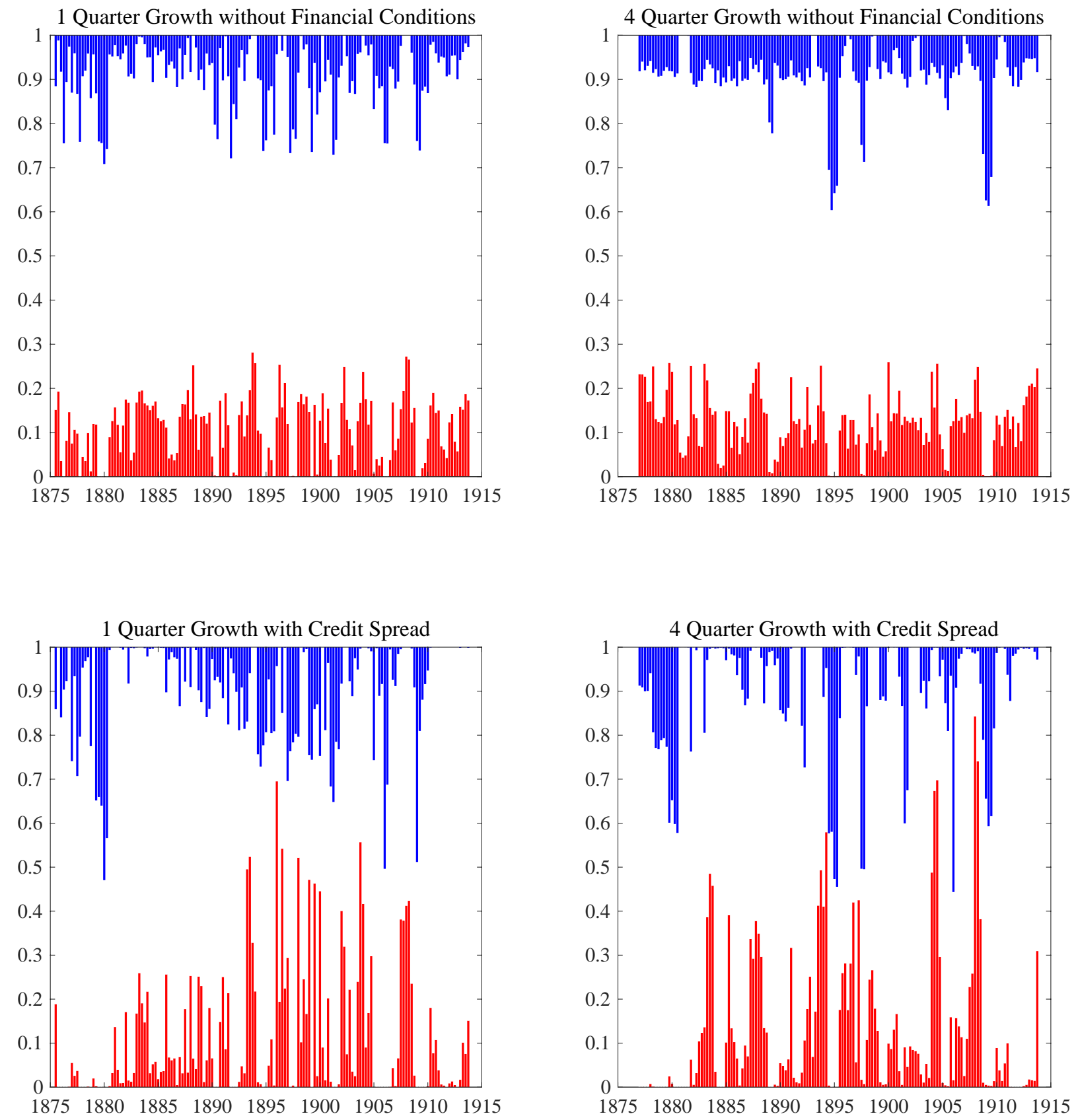

Note: The red bars depict the conditional probability assigned to low output growth events (below the $10^{\text {th }}$ percentile of the unconditional distribution for the sub-sample) and are measured upwards from zero. The blue bars depict the conditional probability of high output growth events (greater than the $90^{t h}$ percentile of the unconditional distribution for the relevant sub-sample) and are measured downwards from one. All specifications use non-Gaussian marginals and non-linear dependence. 
Figure A5b: Probability of Tail Events 1875 - 1913
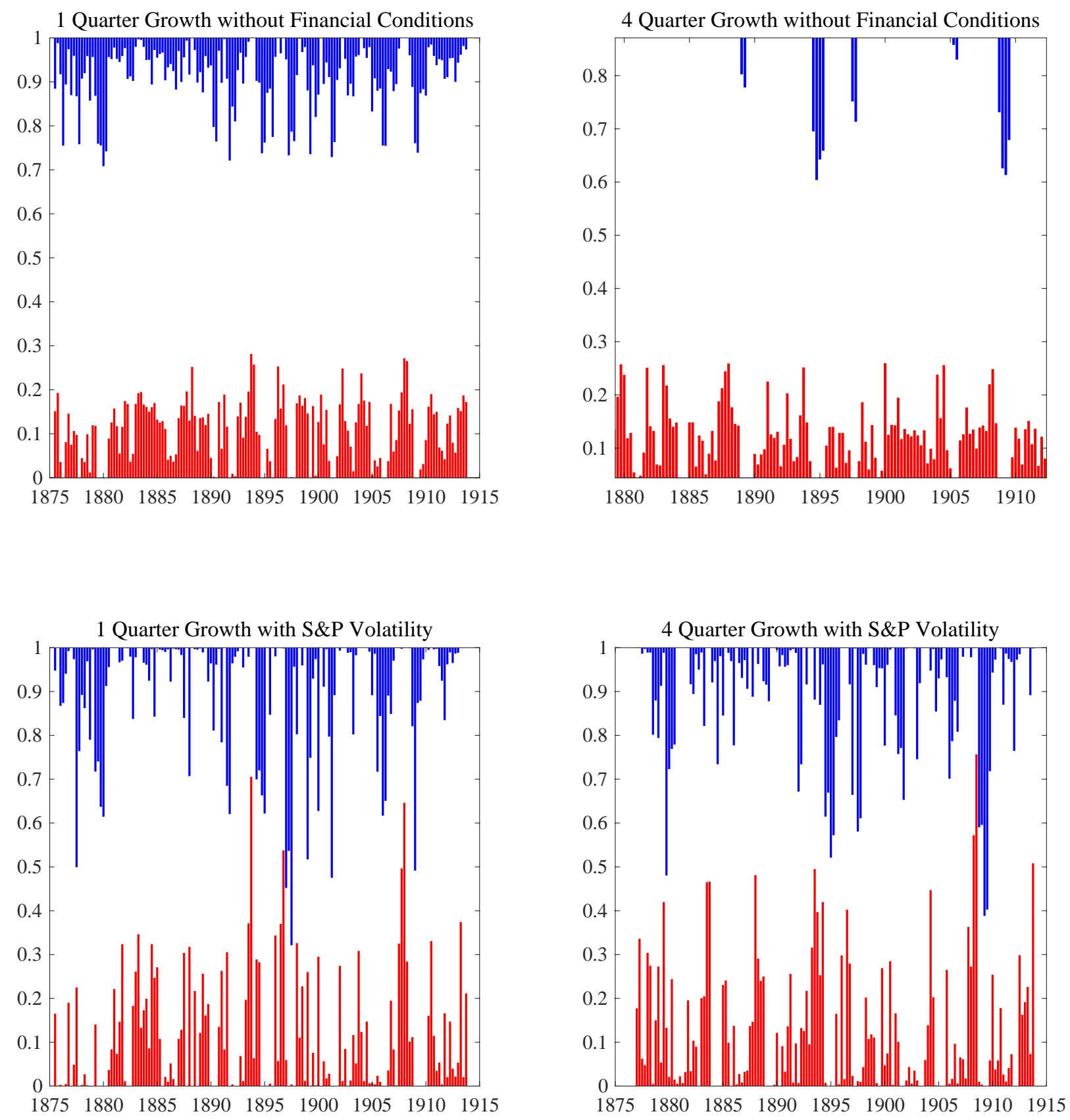

Note: See note to Figure A5a. 
Figure A5c: Probability of Tail Events 1919 - 1941
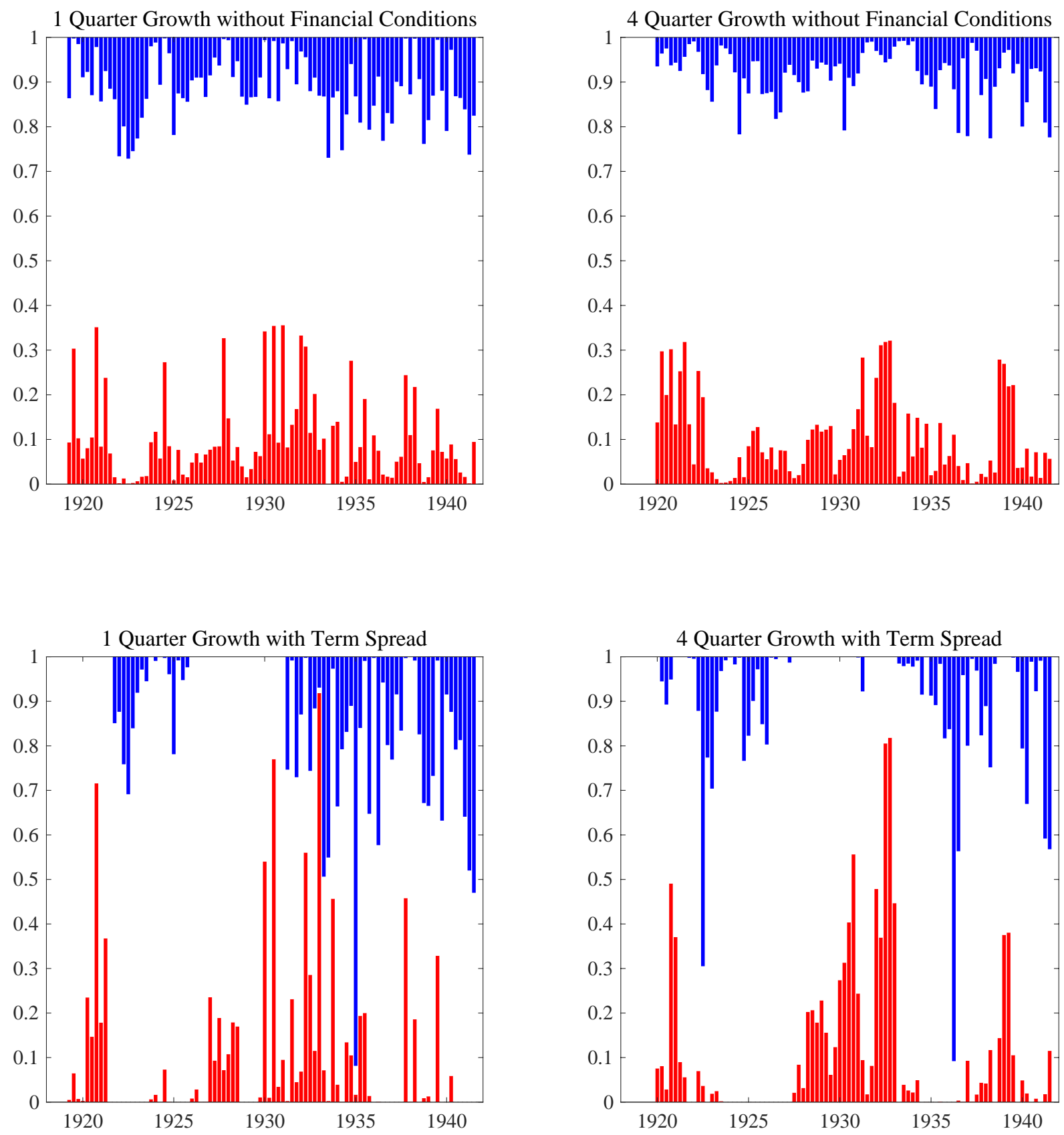

Note: See note to Figure A5a. 
Figure A5d: Probability of Tail Events 1919 - 1941
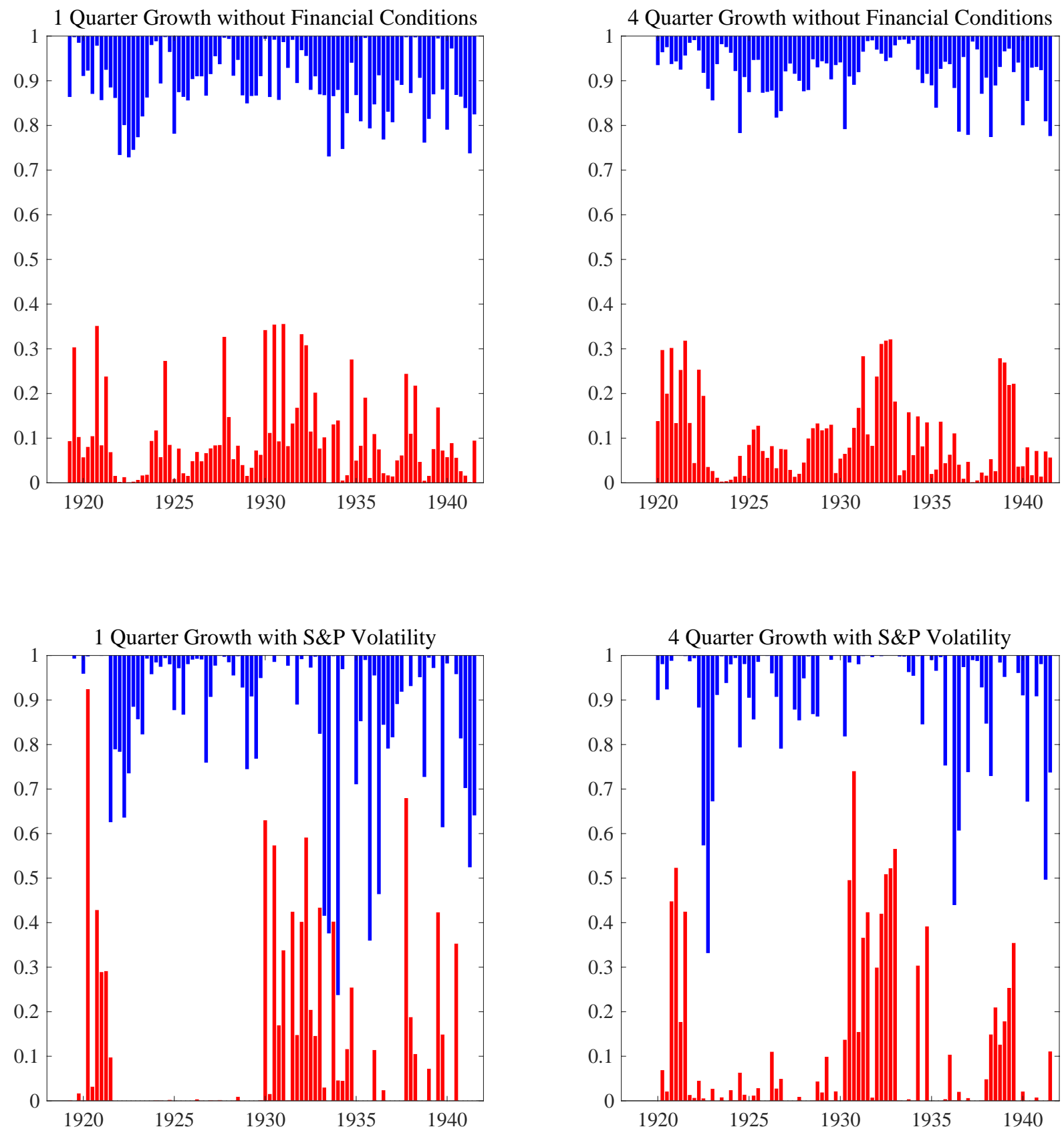

Note: See note to Figure A5a. 
Figure A5e: Conditional Densities 1946 - 1971
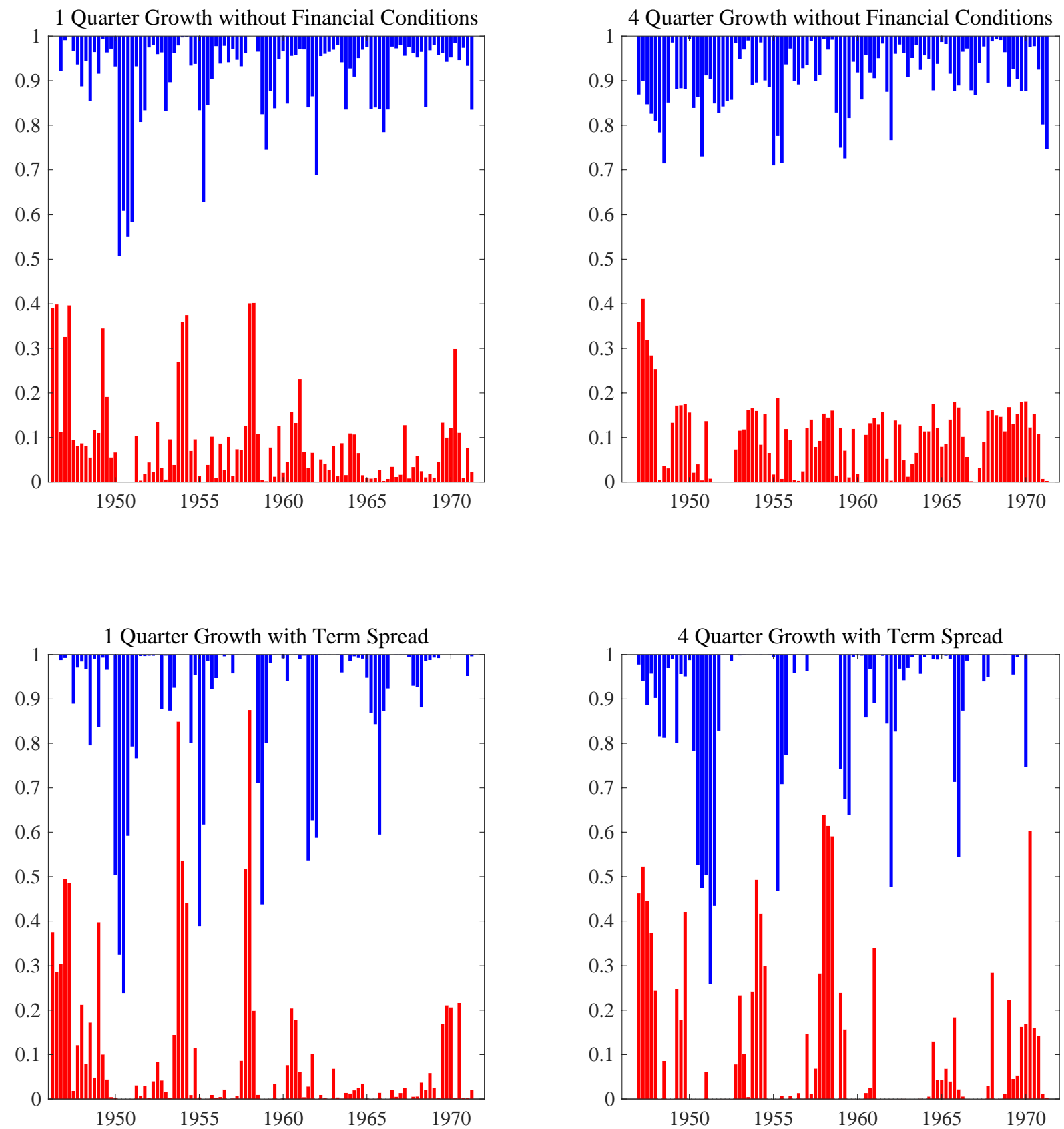

Note: See note to Figure A5a. 
Figure A5f: Probability of Tail Events 1946 - 1971
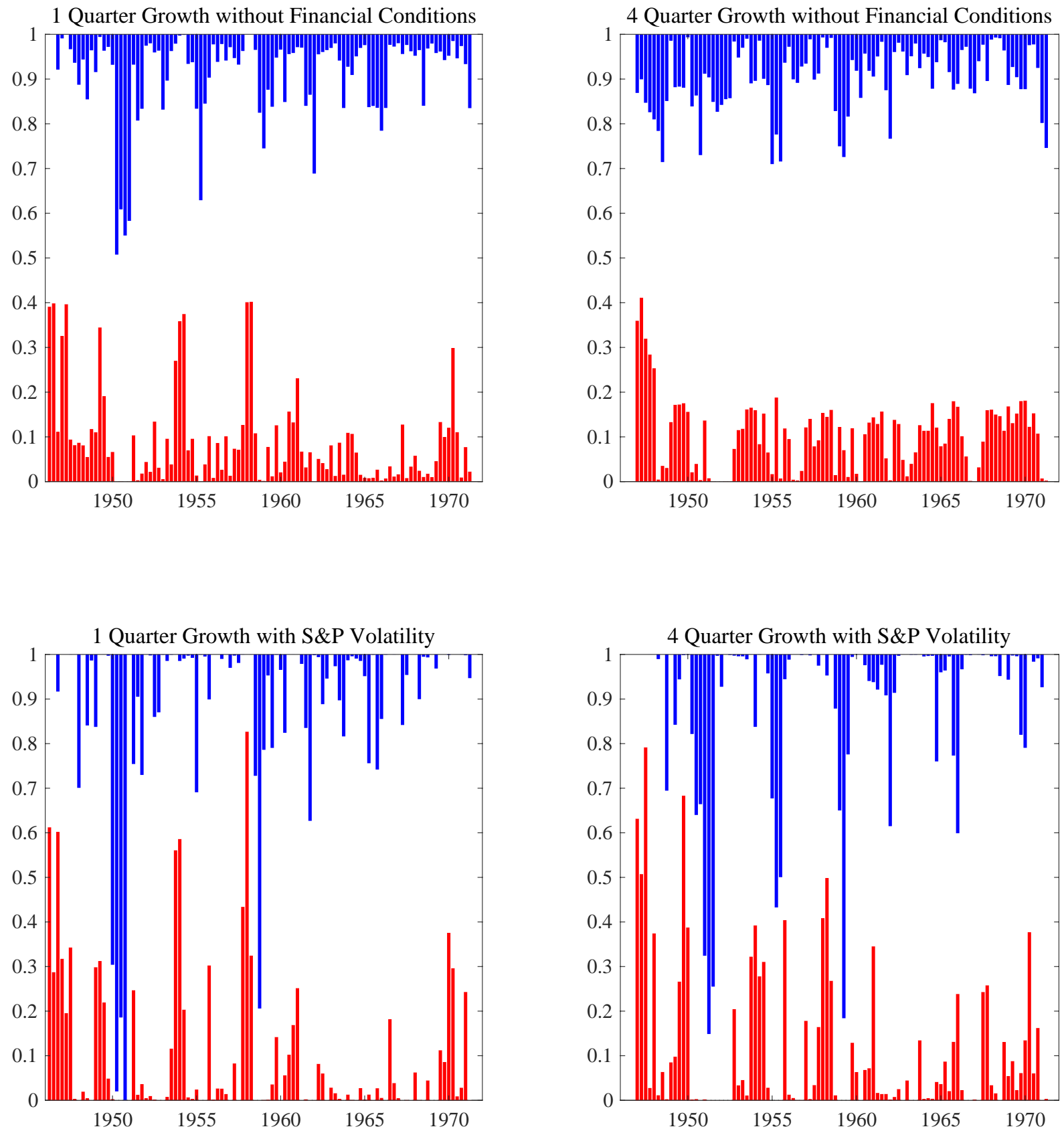

Note: See note to Figure A5a. 
Figure A5g: Probability of Tail Events 1971 - 2017
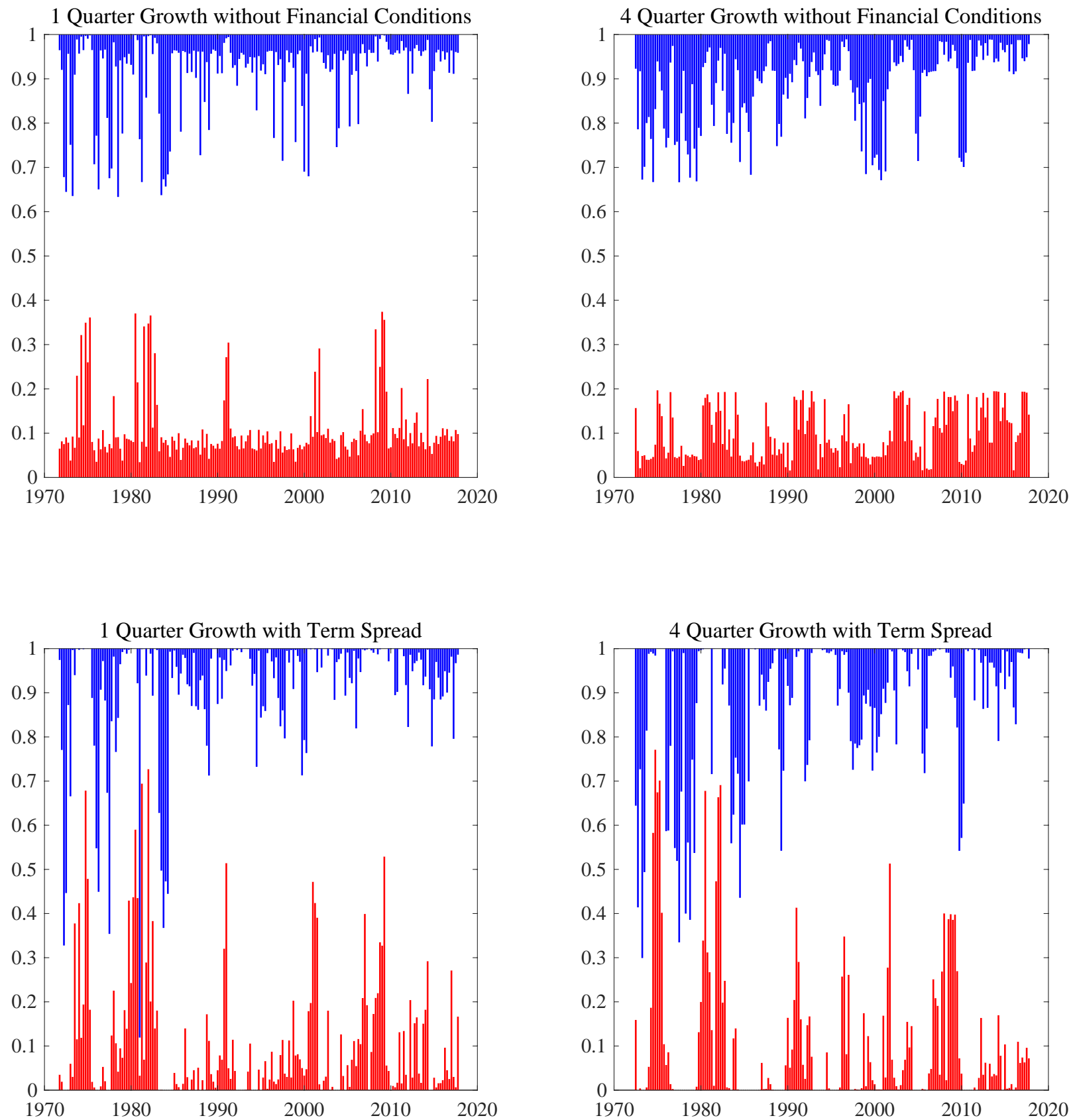

Note: See note to Figure A5a. 
Figure A5h: Probability of Tail Events 1971 - 2017
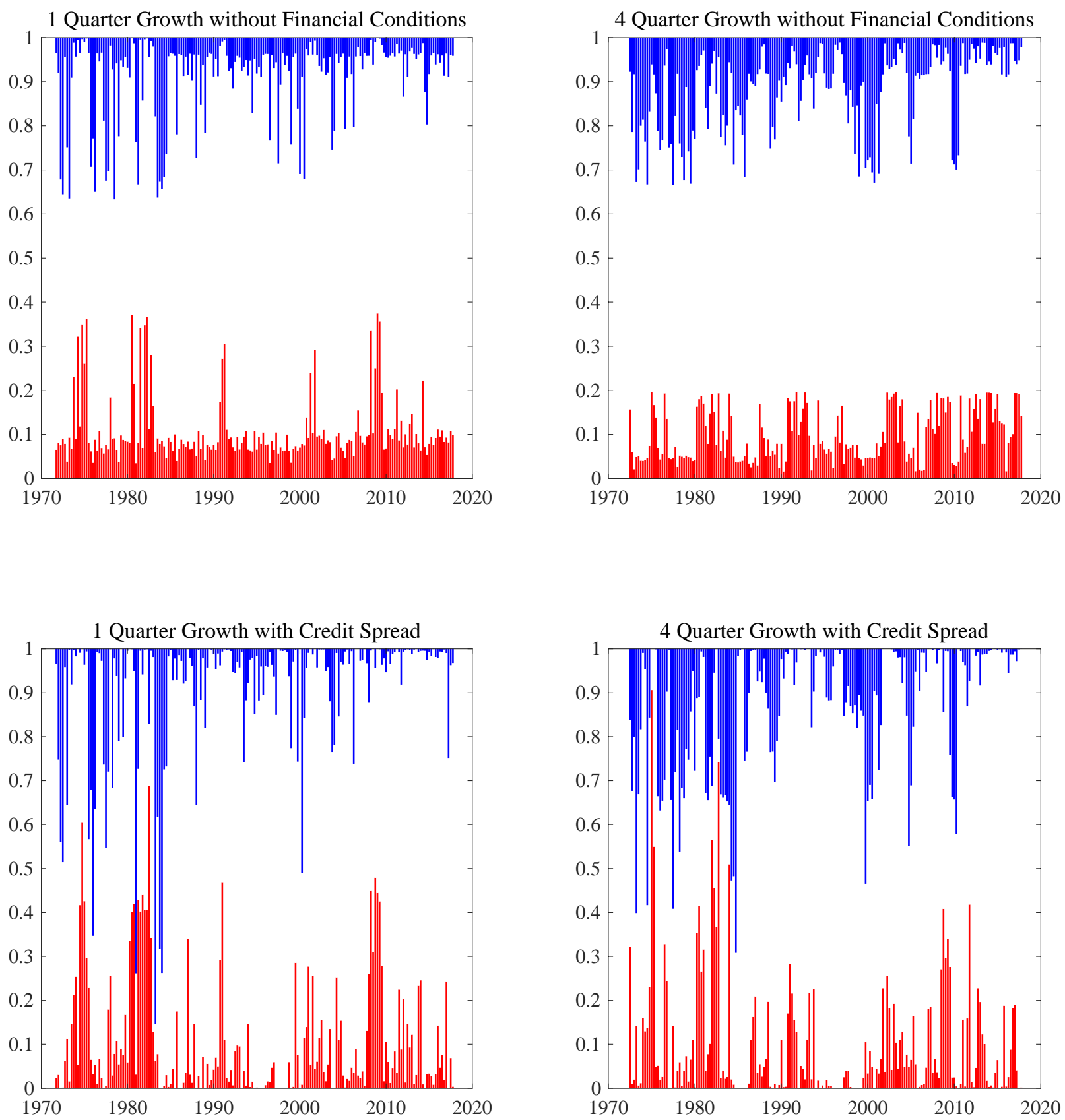

Note: See note to Figure A5a. 
Figure A5i: Probability of Tail Events 1971 - 2017
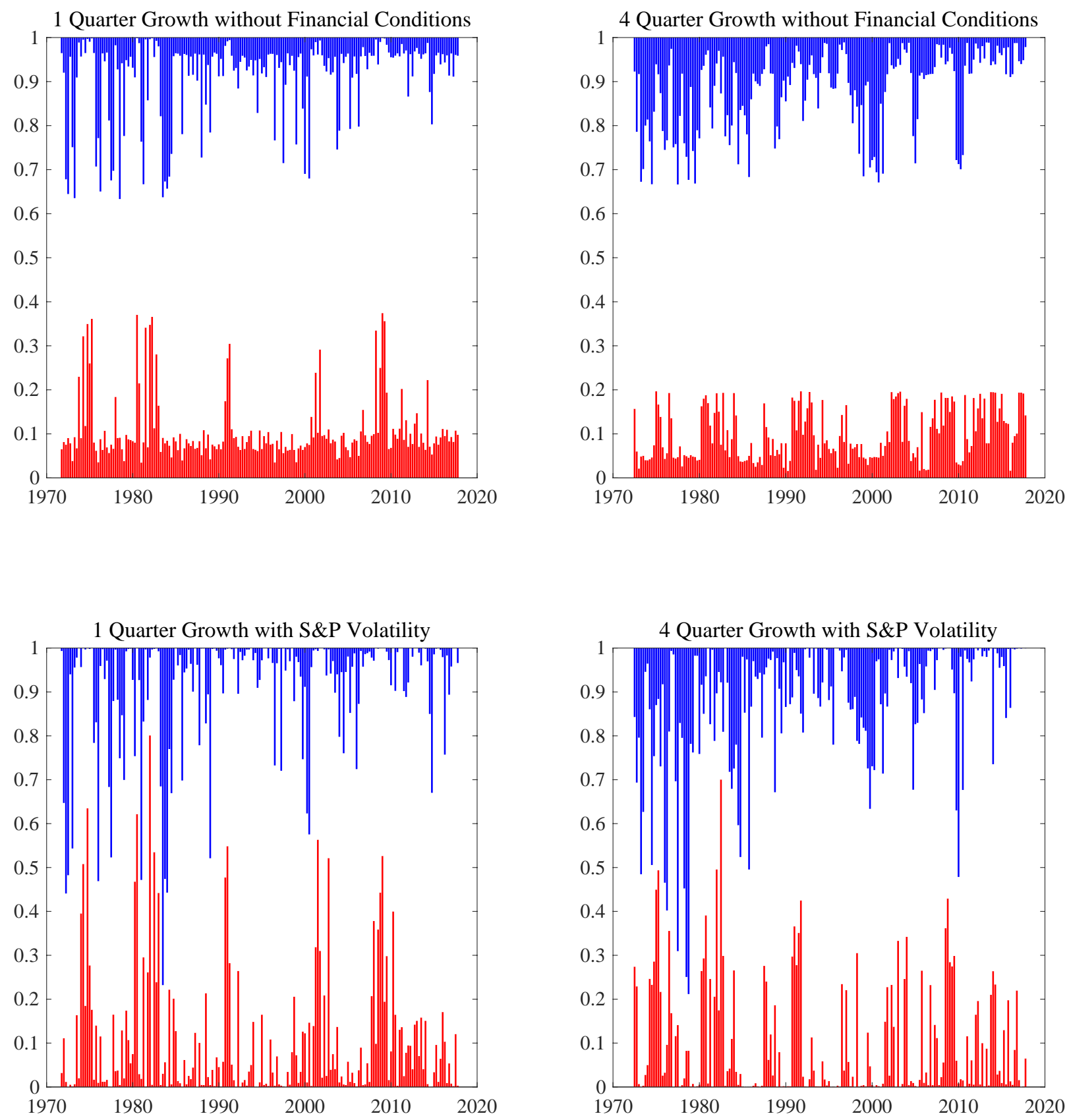

Note: See note to Figure A5a. 


\section{Figure A6: Predictive Densities For Selected Financial Crises}
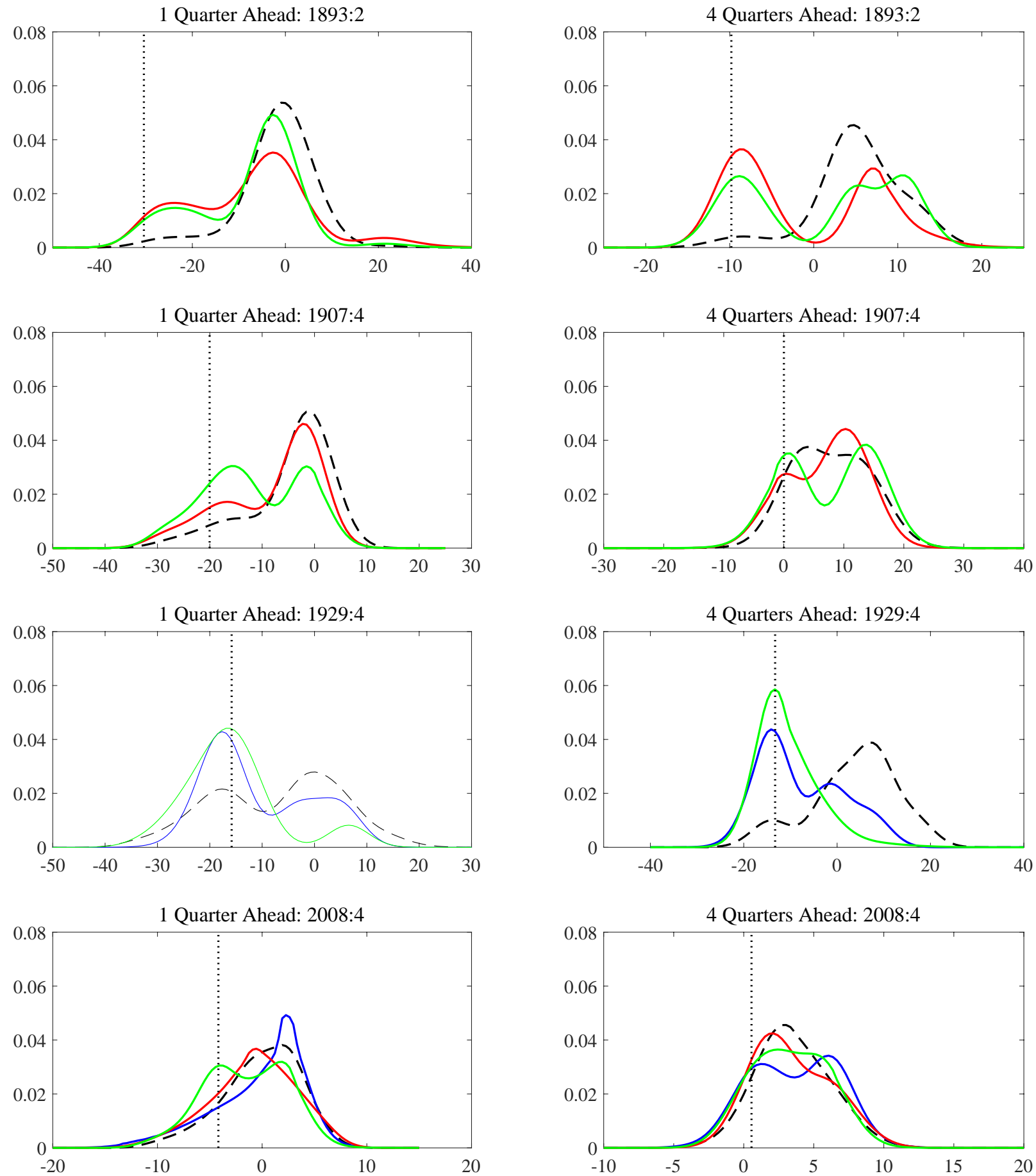

Note: The dashed black line depicts the conditional density based on the specification with non-Gaussian marginals and non-linear dependence, without financial conditions. The solid blue, red and green lines depict the equivalent densities accounting for financial conditions as represented by the term spread, the credit spread and stock market volatility respectively. The left panels display the densities for output growth in the subsequent quarter; and the right panels correspond to the subsequent year. 
Figure A7a: Probability of Tail Events 1875 - 1913
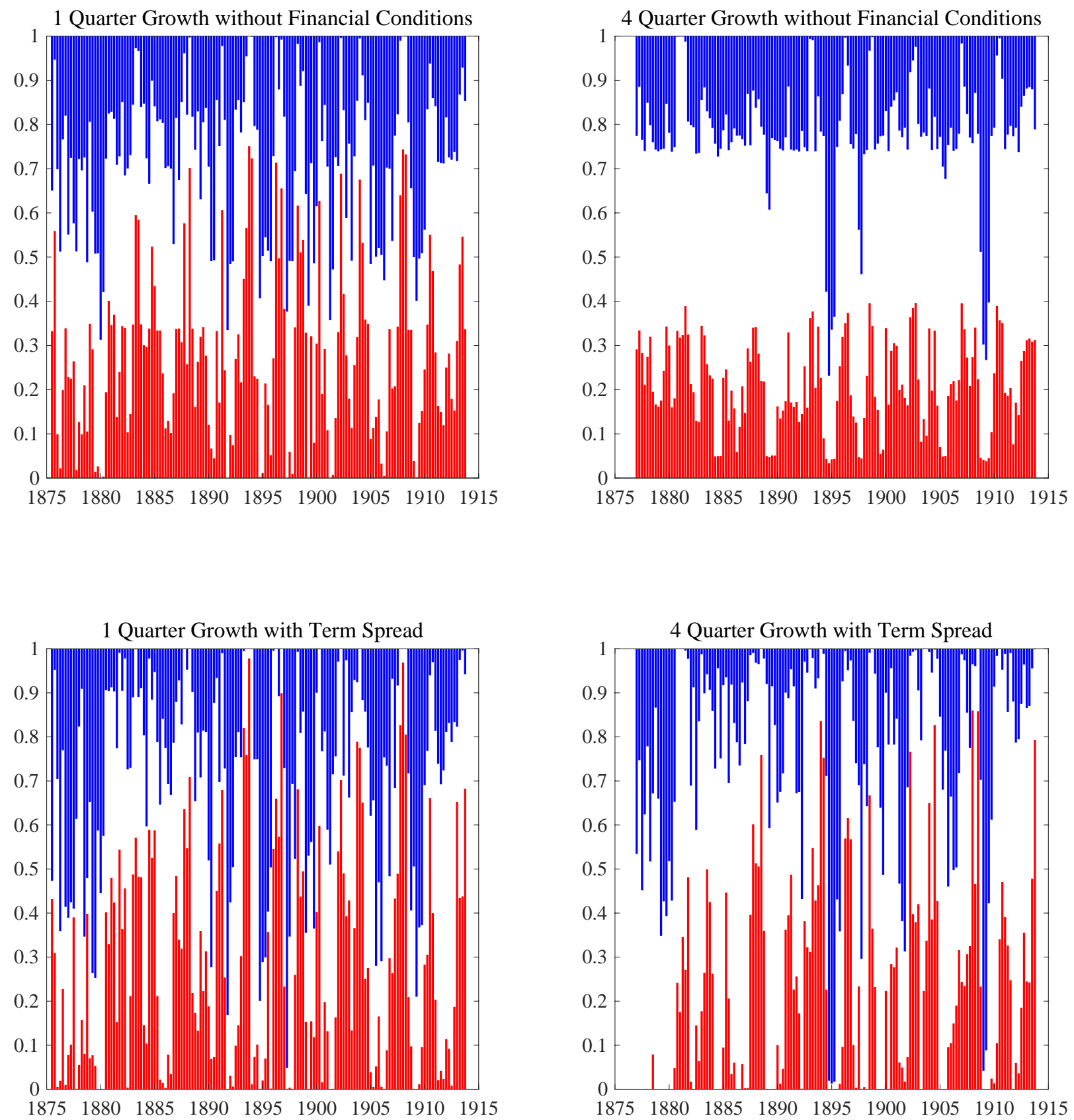

Note: The red bars depict the conditional probability assigned to low output growth events (below zero) and are measured upwards from zero. The blue bars depict the conditional probability of high output growth events (above twice the mean of the unconditional distribution for the sub-sample) and are measured downwards from one. All specifications use non-Gaussian marginals and non-linear dependence. 
Figure A7b: Probability of Tail Events 1919 - 1941
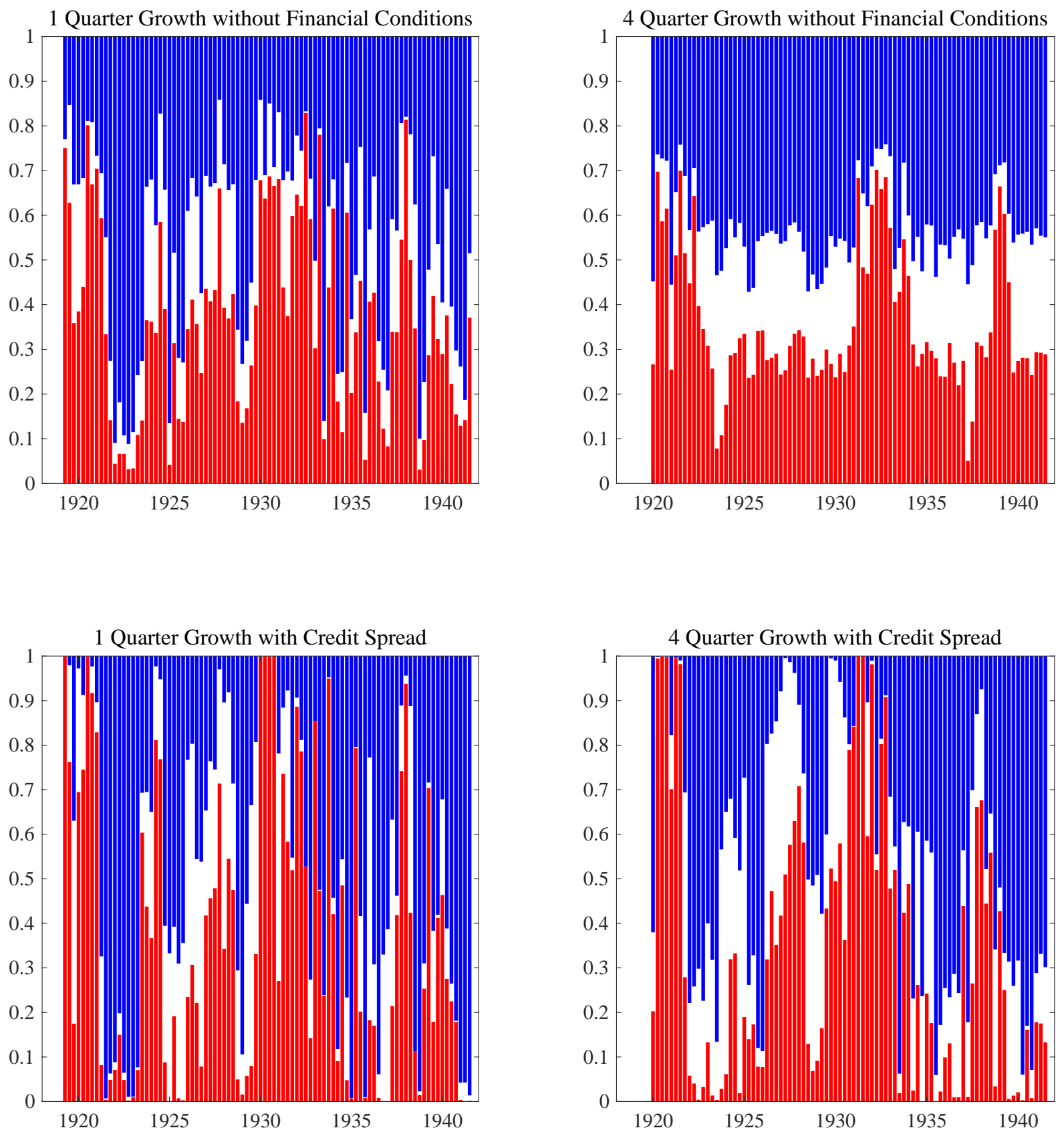

Note: See note to Figure A7a. 
Figure A7c: Probability of Tail Events 1946 - 1971
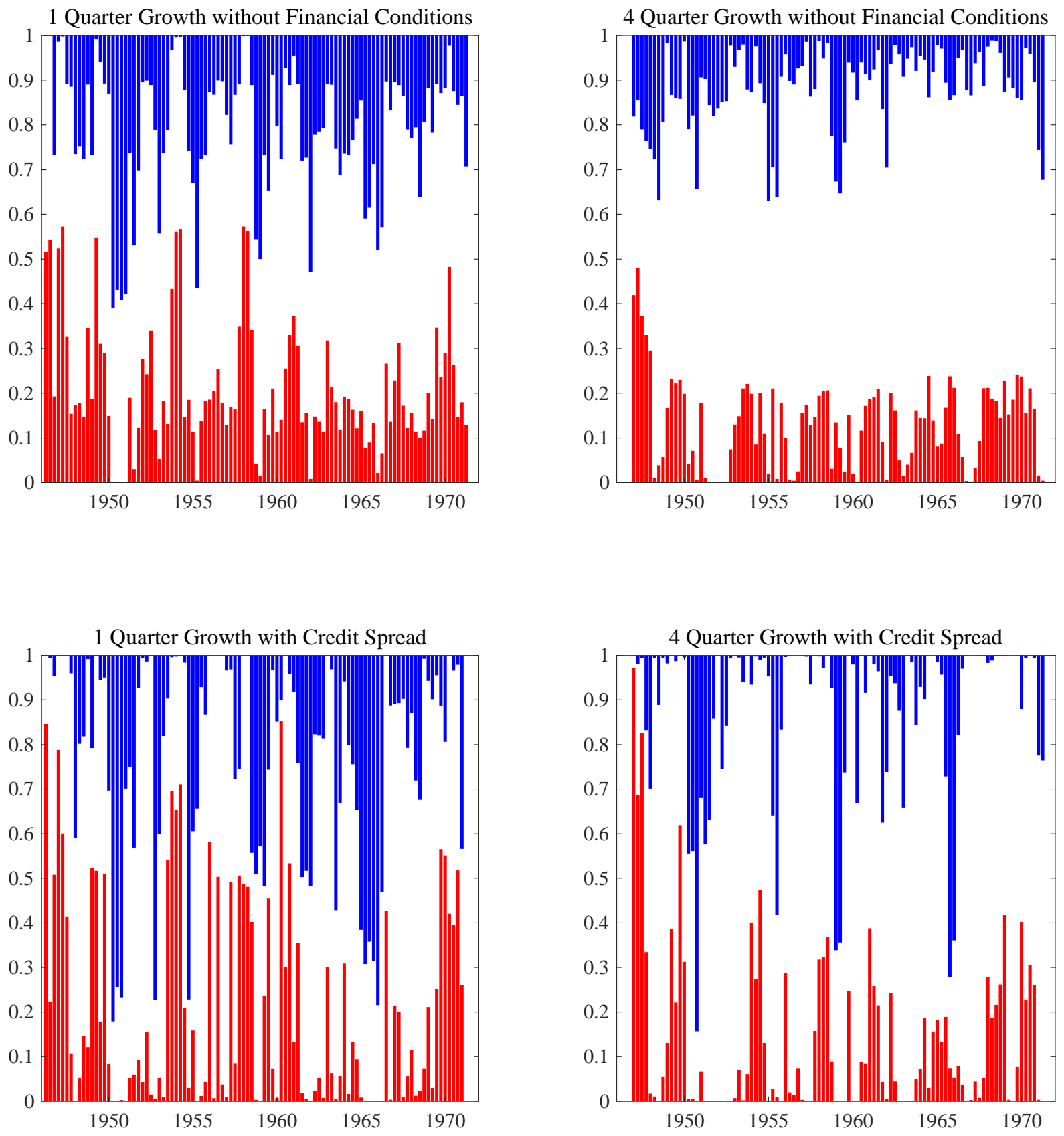

Note: See note to Figure A7a. 
Figure A7d: Probability of Tail Events 1971 - 2017
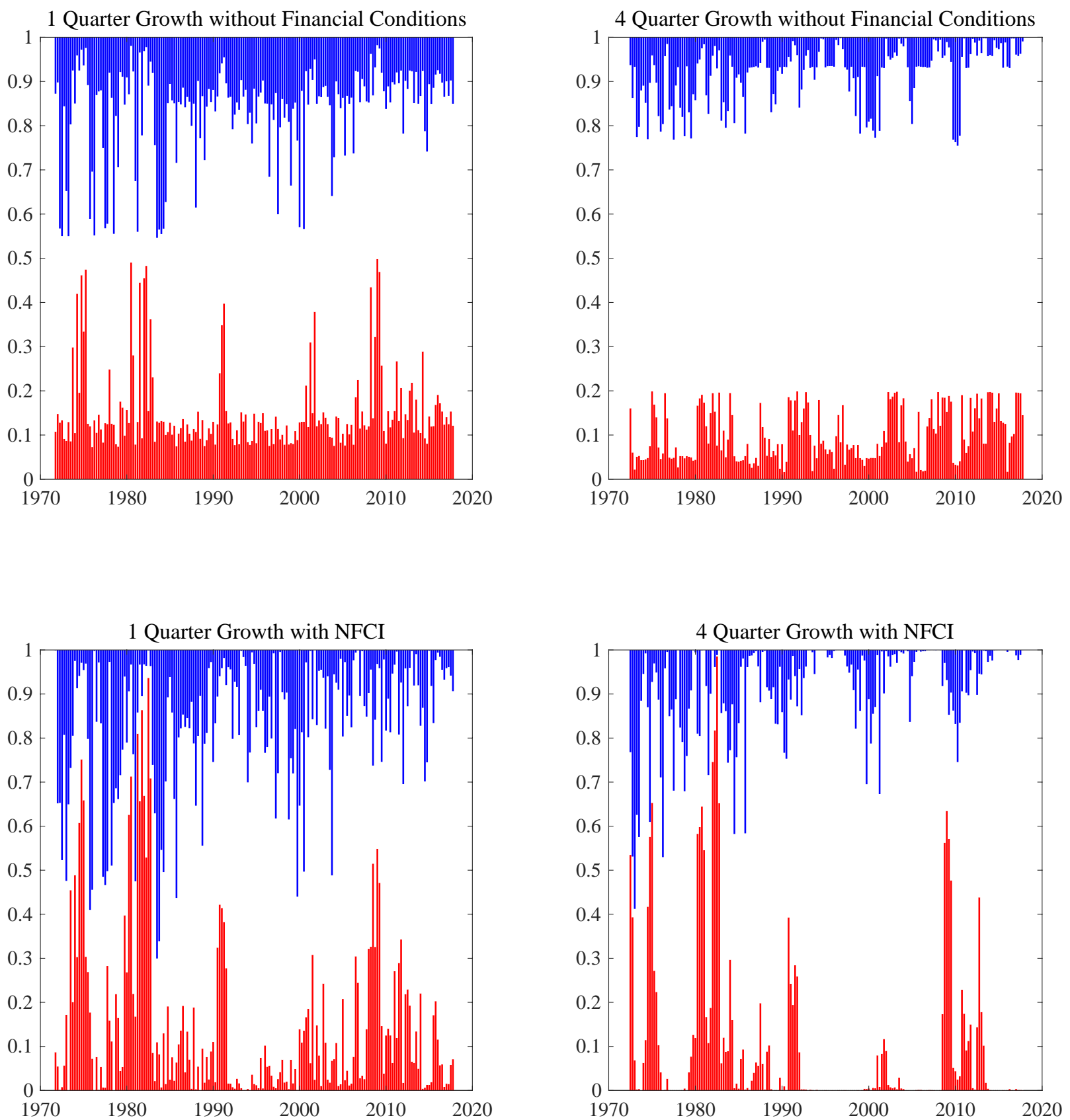

Note: See note to Figure A7a. 